NBSIR 84-2903

\title{
An Investigation of the Uncertainties of the NBS Thermal Voltage and Current Converters
}

\section{F. L. Hermach}

U.S. DEPARTMENT OF COMMERCE National Bureau of Standards Center for Basic Standards

Electricity Division

Gaithersburg, MD 20899

April 1985

Final Report, NBS Contract NB81SBCA0711

Submitted February 28, 1983

Revised August 1984

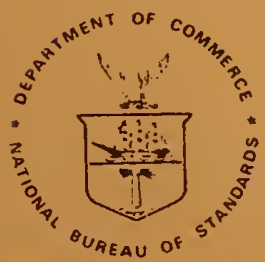

$-Q C$ DEPARTMENT OF COMMERCE

100 DNAL BUREAU OF STANDARDS

.456

84-2903

1985

C. 2 



\section{AN INVESTIGATION OF THE \\ UNCERTAINTIES OF THE NBS THERMAL VOLTAGE AND CURRENT CONVERTERS}

F. L. Hermach

U.S. DEPARTMENT OF COMMERCE

National Bureau of Standards

Center for Basic Standards

Electricity Division

Gaithersburg, MD 20899

April 1985

Final Report, NBS Contract NB81SBCA0711

Submitted February 28, 1983

Revised August 1984 
Summary ......................... 1

I. INTRODUCTION ........................ . . . . . 3

A. Scope of Contract and Reports............ . . . 3

B. Present NBS Standards . . . . . . . . . . . . . 3

C. Ranges and Uncertainties of Calibrations . . . . . . . 4

II. SYNOPSIS OF THE AC-DC STUDY . . . . . . . . . . . 5

III. MJTC'S AND COMPARATOR . . . . . . . . . . . . . . . 7

A. Comparator . . . . . . . . . . . . . . . . . 7

B. Comparison of MJTC's . . . . . . . . . . . 10

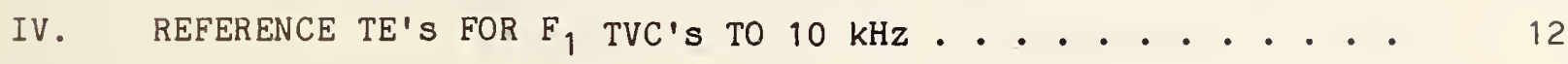

v. EXTENSION OF FREQUENCY RANGE . . . . . . . . . . . . . . . 13

A. Thermoelements ................. . . . . . . 13

B. Thermal Voltage Converters ........... . 15

VI. EXTENSION OF CURRENT RANGE . . . . . . . . . . . . . . 16

VII. EXTENSION OF VOLTAGE RANGE . . . . . . . . . . . . . . 19

VIII. WORKING STANDARDS . . . . . . . . . . . . . . . . . . 23

A. Current Standards . . . . . . . . . . . . 23

B. Voltage Standards .............. . . . 23

IX. THERMOELEMENT REPLACEMENT . . . . . . . . . . . . . . . . . . 24

X. ERROR ANALYSIS - GENERAL REMARKS . . . . . . . . . . . . 25

XI. RANDOM (STATISTICALLY EVALUATED) ERRORS . . . . . . . . . 29

A. MJTC Comparator . . . . . . . . . . . . 29

B. Other Comparators ............. . . . 30

C. Statistical Parameters and Tests . . . . . . . 30

D. Pooled Standard Deviations ............ . . 31

E. Experimental Designs and Tests . . . . . . . . . 31

XII. ESTIMATES OF RESIDUAL SYSTEMATIC ERRORS (Not Statistically

Evaluated) ..................... 32

XIII. COMBINATION OF UNCERTAINTIES . . . . . . . . . . . . 33

XIV. CONCLUSIONS . . . . . . . . . . . . . . . . . . 35

A. Uncertainties of the NBS Standards . . . . . . . 35

B. Calibration Uncertainties . . . . . . . . . 36

XV. RECOMMENDATIONS . . . . . . . . . . . . . . . . . 37

A. Maintenance of AC-DC Standards . . . . . . . . . . 37

B. Quality Control of the Calibration Process . . . . . 38

C. Other Recommendations . . . . . . . . . . . 39 
Table of Contents (Continued)

Page

XVI. ACKNOWLEDGEMENTS . . . . . . . . . . . . . . 4 40

REFERENCES ..................... . . . 4 41

\section{TABLES :}

1 Comparison of Four MJTC's . . . . . . . . . . . 43

2 Differences Between 1981 \& 1976 Evaluations of MJTC's . . . 44

3 Results of Comparisons of FX and FY with MJTC's . . . . . . 45

4 Results of TVC Tests with Twin Resistors . . . . . . . . 46

5 Ampere-Range Comparisons . . . . . . . . . . . 47

6 AC-DC Differences of 1 A to $20 \mathrm{ATE}$ 's.............. 48

7 Results of Evaluation of $\mathrm{FX}_{2}$ and $\mathrm{FY}_{2}$........... 49

8 Comparisons of $\mathrm{F}_{1} \mathrm{TVC}^{\mathrm{S}}$. . . . . . . . . . . . . 50

$9 \quad A C-D C$ Differences of $F_{1}$ TVC's with $\mathrm{FX}_{2}$ and $F Y_{2} \ldots \ldots$

10 Comparisons of Working and Reference Standard TE's . . . . 52

11 AC-DC Differences of Working Standard TE's . . . . . . 53

12 Comparisons of $\mathrm{F}_{7} \mathrm{TVC}^{\mathrm{T}}$. . . . . . . . . . . . . . 54

13 AC-DC Differences of $\mathrm{F}_{7}$ TVC's . . . . . . . . . . 55

14 Pooled Standard Deviations, $s_{p a}$. . . . . . . . . . 56

15 Estimated Limits of Residual Systematic Uncertainties . . . . 57

16 Calculation of Uncertainties . . . . . . . . . . 61

17 Summary of Uncertainties of NBS AC-DC Transfer Standards . . 64

18 Comparison of Estimated Calibration Uncertainties with Present Allowances ............... 65

19 Estimated Uncertainties for Special Tests . . . . . . 66 


\section{Table of Contents (Continued)}

\section{FIGURES:}

Page

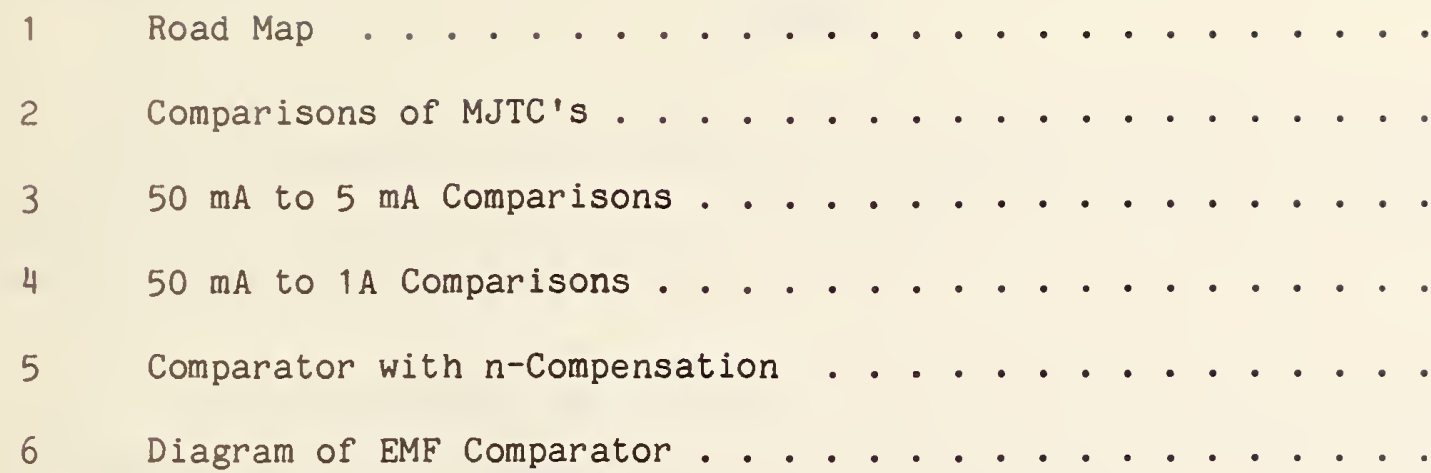

\section{APPENDICES :}

$\mathrm{n}$-Compensation and n-Measurements

$1-1$

2 EMI, Harmonics and DC Offset ............ 2-1

3 Effect of Relay Off-Time . . . . . . . . . . 3-1

4 Effects of Bead Heating . . . . . . . . . . . 4-1

5 Impedances and Substitution Corrections . . . . . . . 5-1

6 Recommendation of the Working Group on the Statement of Uncertainties presented to Comite International des Poids et Mesures ... . . . . . . . . . . 6-1

REPRINTS :

References 2, 3, and 4 .............. R-1 


\section{AN INVESTIGATION OF THE UNCERTAINTIES OF THE \\ NBS THERMAL VOLTAGE AND CURREWT CONVERTERS}

\section{SUEMARY}

This investigation required a complete redetermination of the ac-dc differences of the NBS ac-dc transfer standards, from $20 \mathrm{~Hz}$ to $100 \mathrm{kHz}$. It involved the following steps:

1. Setting up a new and considerably more accurate set of primary ac-dc standards, based on multijunction thermal converters (MJTC's) and a precise emf comparator for comparing pairs of standards,

2. Using them to evaluate 5-mA single junction thermoelements (TE's) and a $10-\mathrm{V}$ thermal voltage converter (TVC), up to $10 \mathrm{kHz}$,

3. Extending the frequency range by complex "bootstrap" techniques that were developed for this, and justifying these techniques,

4. Extending the current and voltage ranges of sets of reference-standard TE's and TVC's by appropriate build-up methods, up to 20 A and from 1 to $500 \mathrm{~V}$,

5. Using these reference standards to evaluate the NBS working standards that serve for calibrating clients' standards,

6. Determining the random errors of each of the above steps,

7. Studying and evaluating residual systematic errors (after known corrections were applied) by special tests and experimental designs, then estimating their individual limits (magnitudes), 
8. Combining these estimates and random errors in a logical way to arrive at estimates of the uncertainties of each of the many reference and working standards (following BIPM Committee guidelines),

9. Carrying out a number of largely mathematical studies relating to the investigation and these sources of ac-dc differences, and, finally,

10. Recommending steps to be taken to improve and insure the continued accuracy of the NBS calibration program for ac-dc differences.

As a result of this work it is believed that the accuracy of the NBS standards and the output of its calibration process for ac-dc transfer are on a much firmer basis than heretofore, with much better documentation.

The studies showed that in certain ranges of voltage, current and frequency considerable improvement in the calibration accuracy can be attained -- factors of 2 to 5 for high-quality single-range TE's and TVC's. Considerable improvement should also be possible for commercial multirange instruments after further study of their stability and other characteristics.

For very special tests, such as international comparisons, accuracies approaching $1 \mathrm{ppm}$ should be possible. 


\section{INTRODUCTION}

\section{A. Scope of Contract and Reports}

In brief, this investigation, performed under contract with NBS, required a theoretical and experimental analysis of the uncertainties in maintaining and using the NBS ac-dc transfer standards for current and voltage measurements at frequencies up to $50 \mathrm{kHz}$, plus suitable documentation of the study. In effect, the contract required a redetermination of the NBS ac-dc transfer standards. This was carried out to $100 \mathrm{kHz}$ for voltage measurements because of frequent calibration requests at the higher frequency.

The contract was completed and the final report was submitted to NBS in February 1983. The report was revised and the appendices were added in August 1984 for publication as an NBS Internal Report.

\section{B. Present NBS Standards}

The basic or primary ac-dc transfer standards at NBS at these frequencies are thermal current and voltage converters, described in [1] and [2]. The working standards used in the calibration of client thermal voltage converters (TVC's) are described in [3]. Papers [2] and [3] are appended to this report for ready reference.

Extensive research was carried out at NBS to establish these standards and verify their accuracy. The basic principles and brief descriptions of the investigations and of the many special tests required for these pioneering developments are given in the references. The work described in [1] (1952) established the ac-dc transfer to $0.01 \%$ (100 ppm) for current measurements from $1 \mathrm{~mA}$ to $20 \mathrm{~A}$ and voltage measurements from 0.2 to $750 \mathrm{~V}$ at audio frequencies. In the work described in [2] (1966) a basic set of 5 to 20-mA single-junction thermoelements (TE's) was evaluated to a rew ppm, and 
two selected TE's were used with special series resistors as thermal voltage converters up to $500 \mathrm{~V}$ to $10 \mathrm{ppm}$. An emf comparator was developed to compare the ac-dc differences of pairs of converters to $\pm 2 \mathrm{ppm}$.

Fifteen comparisons of adjacent or similar ranges are required at each frequency with this primary set of TVC's (called $F_{1}$ ), to verify the build-up (step-up) from 0.5 to 500V. In [3], Williams described a set of TVC's which required only 7 comparisons, thus considerably shortening the time required for routine verification of accuracy. His set (called $F_{7}$ ) is now the working standard for the determination of ac-dc differences of TVC's sent in to NBS for calibration. In a later paper [6] he described a similar set of shunts with two thermoelements (TE's) for evaluating thermal current converters (TCC's). Because of grounding and capacitance difficulties with the shunts, however, TE's are still used as the working as well as the reference standards for TCC calibrations. The contact-type TE's formerly used at $1 \mathrm{~A}$ and above were replaced with insulating-bead types, to eliminate the troublesome direct coupling between the heater and thermocouple circuits. The reference TE's from 25 to $500 \mathrm{~mA}$ were also made to NBS special specifications a number of years ago. Each has a Evanohm ${ }^{1}$ heater, with Evanohm leads from it to the glass press, to minimize ac-dc differences from Thomson and Peltier effects, and from the skin effect of the magnetic leads normally used.

\section{Ranges and Uncertainties of Calibrations}

The following table shows (for ready reference) the stated uncertainties actually available in the calibration of client ac-dc converters at the start of this investigation. It is considered to be very unlikely that the errors would exceed these values.

${ }^{1}$ Certain commercial products or materials are identified in this paper to specify the experimental procedure adequately. Such identification does not imply recommendation or endorsement by NBS, nor does it imply that they are necessarily the best available for the purpose. 


\begin{tabular}{cccccccc}
\multirow{2}{*}{ Frequency } & $\begin{array}{c}2-5 \\
\mathrm{~Hz}\end{array}$ & $\begin{array}{c}5-20 \\
\mathrm{~Hz}\end{array}$ & $\begin{array}{c}20-20 \mathrm{~K} \\
\mathrm{~Hz}\end{array}$ & $\begin{array}{c}20-50 \\
\mathrm{kHz}\end{array}$ & $\begin{array}{c}50-100 \\
\mathrm{kHz}\end{array}$ & $\begin{array}{c}0.1-0.5 \\
\mathrm{MHz}\end{array}$ & $\begin{array}{c}0.5-1 \\
\mathrm{MHz}\end{array}$ \\
Voltage limits (V) & 50 & 100 & $1000^{1}$ & 1000 & 600 & 100 & 100 \\
Current limits (A) & 0.05 & 0.05 & $2.0^{2}$ & 15 & &
\end{tabular}

Uncertainty (percent)3

$\begin{array}{llllllll}\text { Multirange TVCs } & 0.02 & 0.01 & 0.005 & 0.007 & 0.01 & 0.02 & 0.03 \\ \text { Coax single range } & 0.02 & 0.01 & 0.002 & 0.003 & 0.005 & 0.02 & 0.03\end{array}$

TVCs

$\operatorname{TCC}\left(\begin{array}{llllll}.005 & \text { to } 5 \mathrm{~A}) & 0.02 & 0.01 & 0.005 & 0.01\end{array}\right.$

$\operatorname{TCC}(>5$ to $20 \mathrm{~A}) \quad 0.01 \quad 0.02$

1200 volts at $20 \mathrm{~Hz}$, increasing to $1000 \mathrm{~V}$ at $200 \mathrm{~Hz}$.

25 amps at $20 \mathrm{~Hz}$, increasing to $20 \mathrm{~A}$ at $200 \mathrm{~Hz}$.

${ }^{3}$ The lower uncertainty applies at the crossover frequencies. Uncertainties may be increased if the ac-dc differences are large or affected by selfheating.

The larger uncertainties of the multirange converters reflect, in part, the poorer precision and stability of these more complex but very convenient instruments.

\section{SINOPSIS OF THE AC-DC STUDY}

Quite a number of years have elapsed since the comparisons were made which formed the basis for the present accuracy estimates. NBS now has new types of TE's. A series of comparisons of the NBS current converters was started a few years ago, but has not been completed. For these reasons a meaningful study of uncertainties required setting up a new base (primary) group of TE's, as well as carrying out new complete series of interrange comparisons of both the current and voltage standards (reference and working) for extending the ranges from the base (build-up). 
The same emf comparator and the same or new types of TE's could have been used for the base and for these comparisons, as before. However, it was very desirable to use, instead, a group of the newer multijunction thermal converters (MJTC's) and a new comparator, described in [4], (1976), which is also attached to this report. At that time the ac-dc differences (d's) of these 5- to $12-\mathrm{V}$ standards were evaluated to $0.5 \mathrm{ppm}$ up to $10 \mathrm{kHz}$ for the NBS Absolute-Volt Program. However, the converters have not been used since then. For this study the apparatus was set up again with the MJTC's to form an excellent base for directly evaluating the corresponding ranges of the reference $F_{1}$ set of thermal voltage converters (TVC'S), and the TE's used in them as current converters. A considerably better accuracy was thereby attained. Thus this study was based on the new MJTC's as primary standards, with subsequent experimental comparisons with TE's, which were then used for the extensions in frequency and range.

Special studies and tests were made to extend the frequency range from 10 to $100 \mathrm{kHz}$ for voltage measurements and to $50 \mathrm{kHz}$ for current measurements. The d's of the 5-mA reference TE's from all known causes except bead heating should theoretically be independent of frequency from $1 \mathrm{kHz}$ up to at least $100 \mathrm{kHz}$. To buttress the theory they were compared with other TE's of different types and construction, which should also be independent of frequency in this range. As described later in this report, the agreement was excellent.

Resistors of calculable reactance are used with a pair of the reference $T E ' s$ to form the voltage ranges of the $F_{1}$ reference set. In the 1966 evaluation [2], agreement of the 5 to $50 \mathrm{~V}$ ranges, where the computed reactance errors were negligible, formed the basis for assigning d's. Since then evidence of dielectric loss effects has been found. To evaluate them the 10-V range was compared with TVC's containing resistors of measured ac-dc characteristics, and with two of the NBS 30-MHz TVC's [5]. These rf TVC's have carbon-film resistors, with agreement in d's to $0.1 \%$ out to $40 \mathrm{MHz}$. The results showed that there was indeed an ac-dc difference in the 10-V Fq resistor, but that it was less than $6 \mathrm{ppm}$, even at $100 \mathrm{kHz}$. 
A block diagram of these major steps in the evaluation of the NBS ac-dc reference and working standards is shown in figure 1.

Analyses and tests were also made of the important problem of accurately deducing the d's of the many ranges of the $F_{1}$ and $F_{7}$ TVC sets when the TE's are replaced, without retesting all of the ranges. Many other analyses were carried out to evaluate systematic errors from known sources in TE's and in the comparison of TE's. Suitable statistical analyses of random errors were also carried out, and some additional sources of systematic error were discovered.

\section{MJTC'S AND COMPARATOR}

\section{A. Comparator}

For this study the precision MJTC comparator and associated equipment were set up in the NBS AC-DC Laboratory, room A144, MET, which has excellent temperature stability, with a typical range throughout the day of $\pm 0.6{ }^{\circ} \mathrm{C}$. A simplified diagram of the comparison circuits and a description of the procedure for comparing two MJTC's are given in [4]. Briefly, with a resistance divider a fraction of the higher emf of one of the MJTC's is opposed to the full emf of the other. The small differences are read on a nanovoltmeter (nVM), with ac, dc direct, dc reversed, and ac, successively applied to the two heaters at equal time intervals (to minimize the effects of drifts). From these the difference between the two d's is calculated.

Several important changes and improvements were made so that the comparator could also be used for TE's. Brief descriptions follow.

(1) In place of oil immersion for the standards, the metal tank was thermally lagged. This was found to be adequate. 0il immersion would have been impracticable for many of the NBS standards. 
(2) When the two TE's have equal $n ' s,(n=I \Delta E / E \Delta I$, where $I$ is the heater current and $E$ the output emf), the steady-state balance is independent of small changes in the ac and dc supplies, greatly reducing the uncertainty in the measurement and the need to keep the emf of one of the TE's constant during a determination. However, $\mathrm{n}$ may range from 1.6 to 2.0 for TE's, so that some of these advantages are lost. A simple method of compensating the comparator for different $n^{\prime}$ 's was developed. It is described in appendix 1. With this modification the basic equation for comparing two TE's is $\Delta=\mathrm{d}_{\mathrm{T}}-\mathrm{d}_{\mathrm{S}}=\left(\mathrm{N}_{\mathrm{d}}-\mathrm{N}_{\mathrm{a}}\right) / \mathrm{n}_{\mathrm{S}} \mathrm{E}_{\ell}$, where $\mathrm{T}$ refers to the $\mathrm{TE}$ with the higher $\mathrm{n}$, to which the Lindeck potentiometer shown in figure 6 is connected, and $S$ to the other. $\mathrm{N}_{\mathrm{d}}$ and $\mathrm{N}_{\mathrm{a}}$ are the average readings of the nVM with dc and ac applied to the heaters, respectively, and $E_{\ell}$ is the lowest emf of the two TE's. With $\mathrm{N}$ in $\mathrm{nV}$ and $\mathrm{E}$ in $\mathrm{mV}, \Delta$ is in $\mathrm{ppm}$.

(3) An operational-amplifier integrator and timer (called an "averager") was designed and built to integrate the $1-V$ output of the nVM for $10 \mathrm{~s}$, and display the average on a DVM. This significantly reduced the uncertainty from Johnson and nVM noise, and greatly aided the operator. Typically, repeated $10-\mathrm{s}$ averages fluctuated by less than $1 / 10$ th of the peak-to-peak noise seen in $10 \mathrm{~s}$ on the nVM.

(4) The 11-k $\Omega$ input resistance of the 3-stage divider was reduced to $1 \mathrm{k} \Omega$, by switching out the first stage when two single-junction $T E^{\prime} \mathrm{s}$, with their low output resistance, were compared. This improved the performance of the nVM and reduced the Johnson noise of the circuit. The peak-to-peak noise in $10 \mathrm{~s}$ was about $15 \mathrm{nV}$, about 3.5 times the calculated Johnson noise. This is $1.5 \mathrm{ppm}$ of the typical $10 \mathrm{mV}$ rated output of a $\mathrm{TE}$, and $5 \mathrm{ppm}$ with the 3-mV output encountered in interrange comparisons.

(5) Adequate shielding and grounding are very important. All ground leads were connected to the grounded metal tank in which the TE's were placed. A bypass capacitor was connected between each lead and the tank, to reduce any lead-borne interference. Although there was no direct evidence 
that it was significant, electromagnetic interference (EMI) can be particularly troublesome at TV frequencies, where leads are near a quarter wavelength and so pick up electromagnetic energy efficiently.

A simple direct test was devised to see if EMI caused significant errors. With the TE's and supplies connected, but with all power supply switches off, a "phantom" ac-dc test is performed, using the formula for $\Delta$ given in IIIA(2) above with the value of $n_{s} E_{l}$ to be used in the actual test. The result should be zero within the random-error limits. As shown in appendix 2, this test is sensitive enough to detect significant errors.

(6) If there are ac components in the input, chopper-type dc electronic nanovoltmeters (such as the one normally used) may be subject to intermodulation effects which can result in false zero-order (dc) outputs. These normal-mode errors were noted in the MJTC study [4], and were reduced by filtering. Careful measurements showed that at the commonly-used frequencies the ac output of a TE is typically less than $1 \mu \mathrm{V}$, and that this is too small to cause a significant error from this insiduous effect.

(7) About midway in this study, a zener-diode protective circuit, discussed in section VII, was installed at the input to the TVC's, to reduce the possibility of burnout from over-voltages.

A detailed diagram of the comparator, with these changes, is shown in figure 6. It is quite similar to the comparator shown in figure 8 of [3], attached, and the same precautions in its construction, described in [3], were taken. The first stage of the divider is set at zero when the Read 2 position of the switch is used. Shielding, polarity markings, and the fine controls of the Lindeck potentiometer, are not shown. Correct polarity is assured if the nVm goes upscale (more positive) with the set key down when the input to the TVC's or TE's is increased.

The conventional definition of ac-dc difference of a TE is $\mathrm{d}=\left(I_{\mathrm{a}}-I_{\mathrm{d}}\right) / I_{d}$, where $I_{d}$ is the arithmetic average of the two directions of the applied dc current required to give the same output emf as the rms 
current, $I_{a}$. From this $I_{a}=I_{d}(1+d)$, so that an unknown ac current can be measured directly, if $d$ is known, by observing the emf with $I_{a}$ applied and measuring the two polarities of do current which give the same emf. The same formulas apply for TVC's, with V replacing $I$.

In a recent paper, however, [7] Inglis has shown that this definition is in error if the $T E$ has a proportional de reversal difference, $p=\left|I_{1}-I_{2}\right| / I_{d}$, with $E_{1}=E_{2}$, where the subscripts, 1 and 2, refer to the two polarities. He has proposed a new definition, $d_{i}=\left(I_{a}-I_{d}\right) / I_{d}$, where $I_{d}=I_{1}=I_{2}$ and $\left(E_{1}+E_{2}\right) / 2=E_{a}$. It is free of this error, but can be awkward to use directly, for ac measurements. The difference between the two definitions is dependent upon a number of factors. However, for a square-law TE in which $p$ is well behaved (approximately independent of $E$ ), the difference between the two definitions is only about $3 p^{2} / 8$. If $p=5 \times 10^{-4}$ (rather large for a precision $\mathrm{TE}$ intended for ac-dc measurements) the difference is less than $0.1 \mathrm{ppm}$.

At NBS the primary standards are the MJTC's, with p's much less than $1 \times 10^{-4}$. The other TE's are evaluated by the steps shown in figure 1, with the conventional definition of $d$.

Additional analysis shows that, with the conventional definition, there are small error terms which should be added to the basic formula for the NBS emf comparators when TE's having large dc reversal differences are compared. However, they are about the same magnitude as the $3 p^{2} / 8$ term just given, and are therefore also negligible for the NBS TE's, which have small and well behaved p's.

\section{B. Comparison of MJTC's}

The ac-dc difference of 4 MJTC's were carefully compared at 6 to 10 volts (limited by voltage ratings) at $30 \mathrm{~Hz}, 1 \mathrm{kHz}$ and $10 \mathrm{kHz}$. These are 4 of the 8 that formed the MJTC base described in [4]. The 4 are of different design and manufacture. This diversity greatly strengthens the confidence in 
standards, because sources of systematic error are more likely to be discovered in such comparisons than by simply repeating measurements on one standard, or comparing like standards.

The results of the evaluations (including repeat tests on different days) are given in table 1, as $d_{T}-d_{S}$ in $p p m$ for the MJTC's identified as $T$ and $S$. The supplementary designations of the MJTC's shown in parenthesis in the figure, are those given in the 1976 paper.

The pooled standard error, $s_{a}$, (standard deviation of the average) for these measurements was $0.16 \mathrm{ppm}$. If the difference between two averages in repeat tests is less than $\sqrt{2} t s_{a}$ ( $t$ is student's $t$ ) we have no evidence (at a given confidence level) that the difference is statistically significant. At a $95 \%$ confidence level for $k(n-1)$ degrees of freedom with $k=19$ (the number of averages in table 1 ), and $n=4$ (the number of determinations in each), $\sqrt{2} \mathrm{ts}_{\mathrm{a}}=1.4 \times 2.0 \times 0.16=0.45 \mathrm{ppm}$. None of the differences between averages was as large as this.

The results of these measurements at each of the 3 frequencies are shown more vividly in figure 2. Here the number near the head of each arrow is $d_{T}-d_{S}$, where $T$ is the MJTC at the arrowhead. The best estimate for $\mathrm{d}_{\mathrm{Gr25-1}}-\mathrm{d}_{\mathrm{G} 44}=\mathrm{M}$ is obtained from the paths between them, giving double weight to the direct path. The best estimate for $\mathrm{d}_{\mathrm{G} 5 \mathrm{C}}$ is obtained by assigning to each pertinent arrow half of the discrepancy between $M$ and the sum of the two arrows involving G5C. Similarly $d_{\text {NPL14 }}$ is determined. (NPL14 could not be used at $10 \mathrm{kHz}$ because of its magnetic lead wires.)

The value assigned to each MJTC, on the assumption that the average of all 4 is zero, is shown to the nearest $0.1 \mathrm{ppm}$ in each circle. G10C was not included in this group because it was compared only with G5C, in early measurements, which were not repeated. 
The same MJTC's were evaluated in 1976 [4] as part of a larger group. Table 2 shows the differences between the present assignments and those of 1976. They are gratifyingly small, indicating the high stability as well as the low ac-dc differences of these thermal converters of different design and construction.

\section{REFERENCE TE's FOR F 1 TVC's TO $10 \mathrm{kHz}$}

The first comparisons of the 10-V reference TVC with one of the MJTC's, and subsequent intercomparisons of TE's, disclosed several problems. The TVC was unstable because of variable contact resistance in its sliding connectors. Some TE's had a peculiar dependence of ac-dc difference upon the ac-dc sequence used, differing by as much as $3 \mathrm{ppm}$ with ac, dct, dc-, ac applied than with ac, dc-, dct, ac applied. Several of the TE's required 15 to $20 \mathrm{~s}$ to come within $1 \mathrm{ppm}$ of the final value after the input was switched, even though the off-time of the relay was less than $0.5 \mathrm{~ms}$.

To overcome these problems simple modifications were made in the TVC connectors and new 5-mA TE's were chosen after extensive screening tests. Each consisted of two special TE's with Evanohm heaters, welded to the copper skin of the CuNi lead-in wires to reduce thermoelectric errors. ${ }^{2}$ The two heaters were connected in series, as were the two thermocouples, to obtain a rated output of $12 \mathrm{mV}$. They were temperature compensated by the method used by Williams [3].

Two of them, labeled FX and FY, were compared as both current and voltage converters with two of the MJTC's at $30 \mathrm{~Hz}$, and 1 and $10 \mathrm{kHz}$, using the 5- and 10-V F 1 resistors for the TVC tests. The results are shown in table 3. The third TE, FZ, was compared with FX to $100 \mathrm{kHz}$.

\footnotetext{
2 Later analysis indicated that welding to the copper instead of the core might not greatly reduce the Peltier error.
} 
The measured d's were gratifyingly small. There was no appreciable d from Thomson and Peltier effects in the heaters of any of the three TE's. There were small low-frequency errors at $30 \mathrm{~Hz}$, and evidence of impedance errors (about $1 \mathrm{ppm}$ at $10 \mathrm{kHz}$ ) in the TVC resistors.

To check on possible current-dependent d's at high frequencies, FX and FY were paralleled and compared with FZ at $5 \mathrm{~mA}$, with excellent agreement.

Puzzling drifts of some single-junction TE's led to a study of transients caused by switching the input voltage during an ac-de determination. As shown in appendix 3, if the response of a TE to a step-change in input is a simple exponential with a time constant, $t_{c}$, and the relay off-time in transit is $t_{0},\left(t_{0}<\left\langle t_{c}\right)\right.$ then the proportional change in output emf at a time, $t$, after switching (caused by the off time) is $p=t_{o} / t_{c} e^{a}$, where $a=t / t_{c}$. For the high-speed miniature relays now used, $t_{0}$ is only $0.4 \mathrm{~ms}$. For a TE with a $t_{c}$ of $2 \mathrm{~s}$ (fairly typical) a time $t=10 \mathrm{~s}$ is required to make $p<1 \mathrm{ppm}$. This is comfortably below the $30 \mathrm{~s}$ interval (time before reading $\mathrm{nVm}$ ) now used.

The present relays can be used only up to $200 \mathrm{~V}$ in the circuit, and high-voltage relays have much longer off times. Longer intervals might then be required. Surprisingly, for $t_{0}=4 \times 10^{-3} \mathrm{~s}, t=15 \mathrm{~s}$, only a modest increase. Thus the excessively long drifts of some TE's are evidence of other problems, of unknown causes.

\section{EXTENSION OF FREQUENCY RANGE}

\section{A. Thermoelements}

Because of reactances in the complex structure of the 5- and 10-mA MJTC's, these direct comparisons could not be extended reliably in frequency. The simpler TE's, with their smaller reactance errors, should have wider frequency ranges. To verify this, several 5-mA TE's of different construction and/or manufacture were compared with EX and FY as current converters from $30 \mathrm{~Hz}$ to $50 \mathrm{kHz}$. Two of them were in the original 1966 
primary set. All of them have Evanohm heaters and should have low thermoelectric errors. These were determined from the 1-kHz MJTC tests, and were independent of frequency. The important criterion is that the measured differences between each pair of $T E^{\prime}$ 's should be the same at higher frequencies, giving strong evidence that reactance and other errors were negligible.

Unfortunately the measurements showed no such agreement. At $50 \mathrm{kHz}$ discrepancies of up to $4 \mathrm{ppm}$ from the $1-\mathrm{kHz}$ values were observed. It seemed likely that some of the differences could be caused by leakage current through the insulating bead which fastens the hot junction of the thermocouple of a TE to the center of the heater.[1,2] The bead resistance is a complex function of frequency and of bead temperature and voltage. This was verified by making additional measurements with added resistance between the heaters of the two TE's, which changed the bead voltage of the ungrounded one. (The thermocouple circuit is grounded at the emf comparator.) Apparently the bead resistance of these $\mathrm{TE}$ 's was less at $50 \mathrm{kHz}$ than at 1.6 kHz, where bridge measurements were made earlier (as described in [2]).

Detailed analysis of bead resistance errors, given in appendix 4, led to some surprising conclusions. They showed (1) that bead heating (caused by leakage current from the heater to the thermocouple through the bead) is twice as effective in raising the thermocouple temperature as heating from the heater wire, (2) that if one end of the heater is grounded, no error would be caused by bead heating, and (3) this would be true even if a pair of equal TE's, with heaters in series, is used, as in FX and FY. (In the comparisons with the MJTC's described earlier, FX and FY were at the ground end.)

With this background, the comparisons of pairs of TE'S were made by following a method used by Williams. They were compared as TVC's with two nearly identical $30-\mathrm{V}$ series resistors. One end of each of the heaters of the two TE's under test could then be at ground. Appendix 5C shows that by repeating the measurements with the resistors interchanged and averaging the 
results, errors from small differences in the reactances of the resistors could be largely eliminated, and the TE's could be evaluated as current converters.

The results of these measurements are shown in table 4. Except at $30 \mathrm{~Hz}$, there was no significant frequency dependence of the average of the 6 TE's up to $50 \mathrm{kHz}$, with FX as the reference. Thus there is good evidence that the corrections assigned to FX and FY up to $10 \mathrm{kHz}$ by comparisons with the MJTC's could be extended without change to $50 \mathrm{kHz}$.

TE's are subject to low-frequency errors (1) caused by failure to integrate the heating effect of the ac current wave correctly. For FX, $d=+0.9 \mathrm{ppm}$ at $5 \mathrm{~mA}$ and $30 \mathrm{~Hz}$. From these tests, $30 \mathrm{~Hz}$ values could be assigned to all of the other TE's.

\section{B. Thermal Voltage Converters}

As shown in [2], the ac-dc difference, $d_{v}$, of a TVC is $d_{v}=d_{t}+d_{c}$, where $d_{t}=\left(Z_{t}-R_{t}\right) / R_{t}$ and $d_{c}=\left(I_{a}-I_{d}\right) / I_{d}$ for the same output emf.' In these equations, $\mathrm{Z}_{t}$ is the magnitude of the ac impedance, defined as the ratio of the input voltage to the current, I, through the ungrounded input of the TE heater, $R_{t}$ is the dc resistance, and $I_{d}$ is the dc current (average for the two directions) required to give the same output emf as $I_{a}$. Note that $Z_{t}$ includes the impedance of both the TE and the series resistor, and that the equation for $d_{t}$ is a perfectly general one.

Preliminary comparisons of the mid-ranges of the TVC's to evaluate high frequency effects disclosed some disturbingly large differences at 50 and 100 $\mathrm{kHz}$. Calculations showed that the d's caused by the reactances of the series resistors should be much less than $1 \mathrm{ppm}$, and should vary as $\mathrm{f}^{2}$. The observed differences were roughly proportional to f, suggesting dielectric losses as the cause. This supposition was strengthened by the $-1.0 \mathrm{ppm}$ average of $\mathrm{F}_{1} 10-\mathrm{FY}$ and $\mathrm{F}_{1} 5-\mathrm{FX}$ in the comparisons at $10 \mathrm{kHz}$ with the MJTC's 
(table 3). The reactance errors should be completely negligible at this frequency.

To investigate this further, $F_{1} 10-F Y$ was compared with a special TVC that Haddad had studied in 1969 for his GWU Master's thesis under Cutkosky[18]. He had determined the change in impedance (resistive and reactive components) between 1.6 and $16 \mathrm{kHz}$. The comparisons indicated that $F_{1} 10-F Y$ had a $\Delta d$ of about $-1.5 \mathrm{ppm}$.

To carry this to higher frequencies, $F_{1} 10-F Y$ and $F_{1} 20-F X$ were compared with the corresponding ranges of the rf Model C TVC's, which had been evaluated to $40 \mathrm{MHz}[5]$. These TVC's have unspiraled carbon film resistors on cylindrical ceramic bases, as distinct from the spiraled metal-film resistors, of the $F_{1}$ set, with much smaller possibility of dielectric effects.

The results of these several tests were not completely concordant, but all of them pointed strongly to a small but significant $d_{t}$ in $F_{1} 10-F Y$. Later tests showed that the $d_{s}$ of the resistor alone was about $-5 \mathrm{ppm}$ at $100 \mathrm{kHz}$.

\section{vi. EXTENSION OF CURRENT RANGE}

Range extension (build-up) is based on comparisons of adjacent ranges, so that all are determined in terms of a base or reference range. Evaluating the validity of the method is, of course, important, and is often very difficult. For these current ranges, the extension was carried out by two methods, as follows:

(a) From 5 to $1000 \mathrm{~mA}$ two almost identical TE's of one range were first compared. They were then paralleled and compared with each of two TE's of the next higher range, and so on. This is the method used in [2] to compare the 5- to 50-mA TE's. Like that work, pairs of almost identical low-reactance resistors were connected in series with the heaters to assure that the paralleled currents were sufficiently in phase. The analysis of 
appendix 5E shows that the phase angles of the two paths must be equal to better than one mrad. This is readily measured on a commercial impedance comparator, to $100 \mathrm{kHz}$. When the phase angles are negligible, the basic formula for this method, taking into account small differences in the characteristics of the paralleled TE's, is given in appendix II of [2]. This method has the strong advantage that each TE is used at or near rated current, so that the results are not affected by current-dependent errors.

(b) Above $1 \mathrm{~A}$ the heater resistances are too low to be measured on the bridge, and mutual inductances can cause serious errors, so a more complex approach following [1] was necessary. Adjacent-range TE's were compared at the rated current of the lower range. To investigate current-dependent d's, selected ranges were compared with shunted low-range TE's at frequencies low enough so that the reactance errors of the shunts were negligible. Each such comparison was made at two currents. All of the TE's had Evanohm heaters with small thermoelectric effects independent of frequency, so that the extrapolation of current-dependence could safely be made to $50 \mathrm{kHz}$.

The 5 to 50-mA build-up was made with the MJTC comparator in method (a). It was convenient to start with a 50-mA (OF-19) MJTC of Wilkin's later, and very different design[8]. His calculations showed that this MJTC should have less than $1 \mathrm{ppm}$ error up to $100 \mathrm{kHz}$. It agreed with the NPL-14 50-mA MJTC at $30 \mathrm{~Hz}$ and $1 \mathrm{kHz}$ to $0.1 \mathrm{ppm}$, verifying low-frequency concordance. (NPL-14 is frequency-limited because of skin effect in its magnetic leads.)

The results of these 5- to 50-mA comparisons are shown in figure 3 . The numbers near each arrow are $\mathrm{d}_{\mathrm{T}}-\mathrm{d}_{\mathrm{S}}$ in $\mathrm{ppm}$, at the four frequencies shown in the key, where the subscript $T$ designates the TE at the arrowhead. The columns headed "Average" are the averages of the two adjacent TE's, as determined by the two comparisons of that paralleled pair with the two TE'S above them in the figure. The numbers in each block are the d's determined from the intercomparisons (starting with MJTC OF-19), with forced closure of each triangle. (One third of the closure error is applied to each leg.) The 
closure errors were very small, requiring adjustments of only $0.3 \mathrm{ppm}$ or less.

The pooled standard deviation $s_{p a}$ of the average of four determinations of $d_{T}-d_{S}$ was only $0.28 \mathrm{ppm}$. These high precision tests showed excellent performance in these Evanohm-heater TE's. At the low end of this rather long chain of measurements, the values obtained for FZ and FY as current converters agreed with those previously determined from the 5mA MJTC's to $1 \mathrm{ppm}$ at $20 \mathrm{~Hz}^{3}$ and $50 \mathrm{kHz}$, and to $0.5 \mathrm{ppm}$ at intermediate frequencies. Thus systematic errors in the chain were very small indeed.

of particular interest is the excellent agreement at $1 \mathrm{kHz}$ (better than $0.6 \mathrm{ppm}$ ) between the MJTC's and the average of the two $50 \mathrm{~mA}$ and two $25 \mathrm{~mA}$ TE's. ${ }^{4}$ Each of these four single-junction converters has an Evanohm heater and Evanohm stems (lead-in wires) from the heater to the glass press (which serves as a good heat sink). Thus the Peltier as well as Thomson errors of each $\mathrm{TE}$ should be very small. Any residuals would not likely be the same in all of them, nor the same as those in the MJTC's. Thus the agreement provides valuable additional confirmation that the ac-dc differences of the NBS standards caused by thermoelectric effects are considerably less than 1 ppm.

The fast-acting relays controlling the inputs to the heaters limit the high precision MJTC comparison equipment to $50 \mathrm{~mA}$ or $100 \mathrm{~V}$ (not simultaneously). For comparisons at higher voltages and currents, the 1966 comparator [2] was used. The results of the paralleling measurements, method (a), from $50 \mathrm{~mA}$ to $1 \mathrm{~A}$ are shown in figure 4, along with a comparison of one of the 1-A TE's with FG-1, a shunted 10-mA TE described in [6]. The build-up is based on a 0 ppm assignment to the average of the two 50-mA TE's, together with forced closure of each trlangle of tests in the four loops up to $1 \mathrm{~A}$.

3 Extrapolated from the $30 \mathrm{~Hz}$ measurements.

${ }^{4} \mathrm{At} 1 \mathrm{kHz}$ reactance errors and low frequency errors should be negligible. 
For these measurements, $s_{p a}$ was about $1.7 \mathrm{ppm}$, practically independent of frequency. This is not as good as the precision of the MJTC comparator, but is low enough to meet the needs for high-current calibrations. The evaluation of FG-1 with the 1-A TE agreed with Williams' earlier tests [6] to 5 ppm, which is well within the limits of random error for the number of stages involved.

The results of the comparisons of the 1- to 20-A TE's by method (b) are shown in table 5. These air-cooled TE's were made by the Weston Company a number of years ago to NBS specifications. Each has an Evanohm heater, with an insulating bead at the hot junction of the thermocouple. To guard against current-dependent errors, some of them were compared with Williams' shunted TE's. Each comparison was made at two currents, with no consistent evidence of such dependence, so that the results of table 5 could be combined with the values assigned to the 1-A TE to determine the d's of all of the ranges, as shown in table 6 .

For these comparisons $s_{\mathrm{pa}}$ was about $1.7 \mathrm{ppm}$, again not greatly dependent on frequency. The TE's showed rather large d's at $50 \mathrm{kHz}$, probably because of skin effect in the lower ranges, and a peculiar "proximity effect" in the 5 to 20 A ranges. These TE's have heaters of $\mathrm{C}$-shaped cross section rather than tubular. For these ranges $d$ was markedly dependent upon the position of the return lead. [6]

\section{vII. EXTENSION OF VOLTAGE RANGE}

The extension from the base $10-\mathrm{V}$ range of the $F_{1}$ reference set of TVC's up to the $1000-\mathrm{V}$ and down to the $0.5-\mathrm{V}$ ranges required 14 adjacent-range comparisons at each frequency, using the FX and FY TE's with 13 plug-in resistors [2]. Unfortunately, shortly after it was completed a major fault in the ac amplifier burned out both of these excellent TE's. (A lower-range TE connected in series with them as a fuse was more rugged.) Subsequently, 
two unusual sources of error in the ac-dc comparisons were discovered, casting some doubts on the accuracy of the TVC bulld-up.

The first of these was an unexpectedly large error caused by a dc offset voltage, $V_{\text {os, }}$ in the output of the same direct-coupled ac amplifier. Because this was only one direction of dc, 1t would be 1ntegrated incorrectly if the TE had a dc-reversal difference, $g=\Delta E / E$, for the two directions of the same dc voltage. As shown in appendix $2 \mathrm{C}$, the error can be as much as $e=g u / 2$, where $u=V_{O S} / V_{a}$. Thus if $u=1 \times 10^{-2}$ and $g=5 \times 10^{-4}$, e can be $2.5 \mathrm{ppm}$. As shown in $2 B$, this is much larger than the output error $e^{\prime}=q^{2} e_{h}$ caused by a small proportional error, $e_{h}$, in measuring a small harmonic, $v_{h}$, in an ac wave, $\mathrm{v}_{\mathrm{a}}$, where $\mathrm{q}=\mathrm{v}_{\mathrm{h}} / \mathrm{V}_{\mathrm{a}}$. If $\mathrm{q}=1 \times 10^{-2}$ and the error of an rms instrument in measuring the harmonic is $e_{h}=5 \times 10^{-4}$, the error in measuring the combined wave is only $0.05 \mathrm{ppm}$.

The second source of error was an unexpectedly large skin effect in the brass center conductor of the coaxial connectors. At $100 \mathrm{kHz}$, a 6-ppm error was caused by an L-shaped connector sometimes used in front of one of the $0.5-V$ TE's in a comparison. The error decreased, of course, for higher ranges with their higher resistances.

The effects of the dc of fset and troublesome dc transients in the amplifier were eliminated by using a wide-range output transformer. The reference plane in each TVC-comparison was taken as the center of a coaxial "T" connector to which each of the two TVC's was thereafter directly connected.

Two new 5-mA TE's, $\mathrm{FX}_{2}$ and $\mathrm{FY}_{2}$ were assembled to replace $\mathrm{FX}$ and $\mathrm{FY}$. They were evaluated by comparisons with MJTC 644 to $10 \mathrm{kHz}$ and with $\mathrm{FZ}$ from 2.5 to $5 \mathrm{~mA}$ up to $100 \mathrm{kHz} . \mathrm{F}_{1} 10-\mathrm{FY}_{2}$ was evaluated at $20 \mathrm{~Hz}, 1$ and $10 \mathrm{kHz}$ with 644 as a TVC. Because of the importance of the frequency extension, the tests described in section $V-B$ were repeated with $\mathrm{FY}_{2}$. Results of these extensive measurements are shown in table 7 . 
The results of the interrange comparisons of the $F_{1}$ set are shown in table 8 , and the values assigned to each range, with of $F_{1} 10-F Y_{2}$ from table 7 as the basis, are shown in table 9 . In the comparisons each TE is used at two currents, with a series resistor of the given range. If its $d_{c}$ is current dependent, a correction must be applied at each step. Since $d_{v}=d_{t}+d_{c}$ ( see section $\left.v-B\right), d_{v 2}-d_{v 1}=-d_{c 2}-d_{c 1}$, at the two currents corresponding to subscripts 2 and 1 , on the assumption that $d_{t}$ is not voltage dependent.

Unfortunately each of these new TE's has a significant $d_{c}$, which is also somewhat current-dependent, as shown by the results in table 7 . It was difficult to evaluate the current dependence accurately enough, because of the low emf's at the low currents. Any inaccuracy is multiplied by the number of steps from the $10-\mathrm{V}$ base range to the extreme ranges. To reduce the uncertainty, an average value of $d_{c}=d_{c 2}-d_{c 1}$ was calculated from all of the values for both TE's from 1 to $100 \mathrm{kHz}$. Subscript 2 applies at $5 \mathrm{~mA}$ and subscript 1 at either 3.3 or $2.5 \mathrm{~mA}$. This value, $+0.8 \mathrm{ppm}$, was applied uniformly in calculating the $d^{\prime} s$ in table 9 from the comparisons of table 8. The tests with $\mathrm{FZ}$ showed that it was independent of frequency from 1 to $100 \mathrm{kHz}$. At $20 \mathrm{~Hz}$ the values of $d_{c}$ were taken directly from table 7 .

As a check on the accuracy of this average value for $d_{c}$, the ac-dc difference, $d_{S}$, of the series resistor of each range was calculated at $1 \mathrm{kHz}$ by formulas given in appendix 5D, starting with the values of $d_{c}$ of the TE's as given in table 7 , and $d_{v}$ as shown at $0.6 \mathrm{~V}$ in table 9 . At this frequency each $d_{s}$ should be negligible. The computed values ranged from +0.4 to -0.9 ppm, and the average for all voltage ranges, with regard to sign, was only $-0.1 \mathrm{ppm}$. This is an excellent check indeed.

Both $\mathrm{FX}_{2}$ and $\mathrm{FY}_{2}$ have $\mathrm{d}_{c}$ 's which are positive, and so cannot be caused by Thomson effects in their heaters [1]. Unfortunately they also have unusually large negative $d_{h}$ 's for their heater impedances ( -10 and $-12 \mathrm{ppm}$, respectively), calculated from the measurements at $1 \mathrm{kHz}$. These values of $d_{C}$ 
and $d_{h}$ are indicative of large Peltier effects at the heater to lead-in junctions.

At higher frequencies the even larger $d_{h}$ for the $0.6-V$ ranges (TE's alone) are caused by skin effect, which increases the resistance of the magnetic heater leads, causing positive ac-dc differences.

With the $F_{1}$ TVC set these factors require troublesome corrections, but do not invalidate the results, as the correlation at $1 \mathrm{kHz}$ shows. Indeed, they provide excellent checks on the correctness of the basic build-up formulas.

It is to be noted that these TE's cannot be used interchangeably; i.e. $\mathrm{F}_{1} 10-\mathrm{FX}_{2}$ is not known from table 9. Substitution corrections are discussed in section $I X$.

The TVC comparisons below $200 \mathrm{~V}$ were made with the MJTC comparator. Because the thermocouple emf's of the higher-range TE in each pair were low ( $3 \mathrm{mV}$ or less), the precision was poorer than with $10 \mathrm{mV}$ outputs. The pooled standard deviation, $s_{\mathrm{pa}}$, was $0.52 \mathrm{ppm}$. The 1966 comparator was used for the higher voltage ranges, with the original TE'S, FX and FY. To avoid repeating these measurements the substitution formula given in section IX was used to calculate the $d_{v}$ 's with $F_{2}$ and $F_{2}$.

A study of protective circuits to guard TVC's from burnout, sparked by the loss of FX and FY, showed that a simple combination of a pair of back-to-back zener diodes directly in parallel with the TVC's and a 10-mA expendable $T E$ in series with the combination should provide adequate protection against reasonable kinds of over voltages. The zener voltage should be near, but not less than, twice the supply voltage, and there are limits on the zener resistance and the time constant of the fuse (which is why a TE was chosen). The method does work well, and TE's of large dc reversal difference, which are relatively inexpensive, can serve well as fuses, but a CRO across the TVC's is advised, to monitor the wave form. 


\section{WORKING STANDARDS}

\section{A. Current Standards}

The working standards which are used in calibrating thermal converters sent to NBS were evaluated by direct comparison (heaters in series) with the corresponding ranges of the reference converters, in the calibration console normally used for such measurements. This had the advantage of giving direct information on the precision of calibrating TE's as current converters (TCC's). Usually the input line to the working standard was grounded. To check on possible bead resistance errors, some measurements were repeated with the reference standard at ground. (The ground should never be placed on the line connecting the two heaters, because capacitance and leakage currents from the ac and dc sources to ground could cause the two heater currents to differ.)

The results of these comparisons are shown in table 10, and the values assigned to the working standards are shown in table 11. The overall spa was $2.4 \mathrm{ppm}$.

\section{B. Voltage Standards}

The $F_{7}$ set of TVC's for calibrating TVC's sent to NBS was evaluated by adjacent-range comparisons, as in [3], with the calibration console. The results are shown in table 12 .

The 10-V range was compared with $\mathrm{F}_{1} 10-\mathrm{FY}_{2}$, as the basis for the build-up. However, insuring the validity of the build-up process is somewhat more complicated than with the $\mathrm{F}_{1}$ set. Each series resistor is used with both TE's, $F_{7} 1$ and $F_{7}$, to form two ranges (such as 3 and $6 \mathrm{~V}$ with the $800-\Omega$ resistor). Then, since $d_{v}=d_{t}+d_{c}$, the difference between those two ranges is $d_{v 2}-d_{v 1}=d_{t 2}-d_{t 1}+d_{c 2}-d_{c 1}$. Thus $d_{c}$ of each of the two TE's must be evaluated (at the comparison currents if it is current dependent). Since $d_{t}$ depends on the heater of the TE as well as the resistor, any difference between the two $d_{t}$ 's should also be evaluated to determine $d_{v 2}-d_{v 1}$, the correction to be 
applied at each step of the build-up comparisons. This is quite complicated unless the reactance terms are negligible as shown in appendix $5 B$ and discussed in the next section.

Fortunately the $d_{t}$ variations caused by TE's decrease with increasing range. For this build-up of the $F_{7}$ set, $d_{c}$ corrections were evaluated from the comparison of the $6-\mathrm{V}$ and higher ranges at $20 \mathrm{~Hz}$ and $1 \mathrm{kHz}$ (where the efect of $d_{h}$ of each heater is negligible). Since the $d_{s}$ 's of the series resistors are also negligible at these relatively low frequencies, the same $d_{c 2}-d_{c 1}=d_{v 2}-d_{v 1}$ is evaluated in each of these comparisons, where $d_{c 2}$ refers to $\mathrm{F}_{7} 1$ at 1.25 or $1.67 \mathrm{~mA}$, and $d_{c 1}$ to $F_{7} 2$ at $5 \mathrm{~mA}$. The average value is less subject to random errors. For these TVC's each $d_{v 2}-d_{v 1}$ was very small. At $20 \mathrm{~Hz}$ and at $1 \mathrm{kHz}$ the average of all of them at $6 \mathrm{~V}$ or above was $0 \mathrm{ppm}$.

Thus the $d_{c 2}{ }^{-d} c 1$ corrections were negligible at these frequencies. They were taken to be the same at higher frequencies. This assumption was checked to a few ppm by special tests involving the known $F_{1}$ TVC's and the $F_{7} 1$ and $\mathrm{F}_{7} 2 \mathrm{TE}$ 's. Similarly, the $d_{h}$ corrections caused by heater impedances were very small.

The results of the $F_{7}$ build up are given in table 13. They were checked by comparing the $1-V$ and $100-V$ ranges with the same ranges of the $F_{1}$ set. The differences ranged from $2 \mathrm{ppm}$ or less at $1 \mathrm{kHz}$ to $6 \mathrm{ppm}$ at $100 \mathrm{kHz}$, and are generally within the random error limits imposed by $s_{p a}$, which were $1.2 \mathrm{ppm}$ at $1 \mathrm{kHz}$ and $2.8 \mathrm{ppm}$ at $100 \mathrm{kHz}$.

\section{THERMOELEMENT REPLACEMENT}

It is highly desirable to be able to replace a TE in a TVC set without recalibrating all of the ranges. The analysis in appendix $5 \mathrm{~B}$ of the substitution error which may result shows that the ac-dc differences as current converters, $d_{c}$, and as voltage converters, $d_{v h}$ of both the old and new TE's must be known, and the phase angles of the impedances must be less 
than certain specifled limiting values. At NBS, $d_{c}$ and $d_{v h}$ can be measured by comparison with the reference standards, and the phase angles can be determined closely enough with a commercial impedance comparator to $100 \mathrm{kHz}$.

If the phase angles are negligible, the change in $d_{v}$ of a given range of a TVC set when $T E_{1}$ is replaced by $\mathrm{TE}_{2}$ (of the same nominal range and input resistance) is given by

$$
d_{v 2}-d_{v 1}=\frac{R_{h}}{R_{t}}\left(d_{v h 2}-d_{v h 1}\right)+\frac{R_{s}}{R_{t}}\left(d_{c 2}-d_{c 1}\right),
$$

where $R_{h}$ and $R_{S}$ are the nominal values of the heater and series resistances, $R_{t}=R_{h}+R_{S}$, and $d_{v h}$ applies to the heater alone.

It is to be noted that as the voltage range is Increased, the first term becomes negligible and the second approaches $d_{c 2}-d_{c 1}$.

If the phase angles are not negligible or cannot be determined, it is best to repeat the lower inter-range comparisons. Although the phase-angle term is complicated, the analysis shows that the error in neglecting it will be less than $1 \mathrm{ppm}$ if the angles of the two TE's are equal to within $0.1 \mathrm{mrad}$ and the angles of the resistors and the TE's are less than $5 \mathrm{mrad}$.

\section{ERROR ANALYSIS - GENERAL REMARKS}

These are personal views on various phases of error analysis.

(1) There are many sources of systematic errors in these standards and in the comparison process. Practically all are independent. Our estimates of their individual limits, after all known corrections are applied, should then be added in quadrature as the square root of the sum of their squares (rss), following Youden [10]. 
(2) Youden recommends adding the estimate of the random errors (such as $2 s_{a}$ or $3 s_{a}$ ) to the rss of (1) to obtain the total uncertainty.

(3) However, it is evidently desirable to follow the much more recent guidelines of the BIPM Working Group on the Statement of Uncertainties, in accordance with B. N. Taylor's memorandum of August 21, 1981 [11]. This involves adding all of the components in quadrature, including $s_{a}$. They are to be estimated as the equivalent of "standard deviation". For convenience, the BIPM recommendations are reproduced in appendix 6 .

(4) It is generally not feasible to determine a probability distribution for each of these error estimates, even though one feels that the true value may be nearer the center than at the estimated limits. Thus it is safer (more conservative) to assume a rectangular distribution, with limits $\pm b$. The standard deviation, $\sigma$, of such a distribution is $b / \sqrt{ } 3$. C. F. Dietrich, of the British Calibration Service, has shown that if rectangular distributions are combined with each other and with a normal (Gaussian) distribution, the standard deviation of the combination is

$$
\sigma_{c}=\left(\begin{array}{ll}
n & \sigma_{i}^{2} \\
1
\end{array}\right)^{1 / 2}
$$

As a result of his extensive analysis of such combinations he states that [12]

"4.69 Let us look at this another way round. If we know the standard deviations of the Gaussian uncertainties of an item of calibration, and thus the total Gaussian standard deviation, and if we assess the maximum value of each of the estimated uncertainties, and assume that these have a rectangular distribution, we can at once find the total standard deviation of the measurements. We can then state that the probability of an uncertainty lying outside $\pm 2 \sigma$ is less than 0.0455 or the probability of an uncertainty lying outside $\pm 3 \sigma$ is less than 0.0027 , without having to go to all the trouble of calculating the combined distribution and calculating the required probabilities. The correct 
probabilitles will always be less than the corresponding Gaussian ones having the same tolerance limits and standard deviation, but usually by only a small amount."

This very important conclusion provides a simple yet generally realistic method of combining error estimates. It is on the conservative side (i.e., does not underestimate the total).

(5) A very important factor in error analysis is to gain enough knowledge of, and experience with, the measurement process to be able to find the (generally hidden) causes of residual uncorrected errors, and to get reasonable estimates of the error limits. This inevitably takes time.

(6) Because of the difficulty of finding error sources by simply repeating measurements in the same way, it is very desirable, as is well known, to vary the conditions of the measurement and note the effects on the results. The imposed variations should be well beyond the normal limits.

(7) Thus in research to establish the accuracy of physical standards, every effort should be made to vary or change the associated measuring equipment. In contrast, as Churchill Eisenhart has emphasized [13], the use of standards in calibrating others should be considered as a measurement process which must be kept under statistical control, with only specified ranges of the variables allowed. The measurement process should be well documented to insure this through the years.

(8) It is also very desirable in the research phase to make measurements with different standards and different comparison apparatus, whenever possible. We know that having different TE's made in different ways by different manufacturers can give a lot of confidence in the results if all of them agree, and can help disclose sources of error if they do not. 
(9) "Round-Robin" tests of three or more TE's or TVC's of the same range, and other more complex experimental designs, can also be very valuable means of disclosing systematic errors, as is well known.

(10) As figure 1 shows, there are several steps in the determination of the accuracy of the final or working standards of ac-dc difference. It is well to note that, if corrections are applied at each step for the known (determinate) errors, the estimates of remaining residual errors (these are estimates not errors) are combined as the square root of the sum of their squares (rss), not added directly. There is no need for the crippling "accuracy ratios" or factors of $3 / 1$ to $10 / 1$ in each step, as so of ten specified.

(11) Operator mistakes and certain equipment malfunctions are not amenable to analysis and inference, but can be major sources of error. For established calibration programs, where the volume of work justifies it, they can be reduced by computer control and computer verification of important parameters. They can also be reduced by repeating critical calibrations with a second operator and a second calibration console, and by the calibration of check standards in an adequate quality-control program. Having a second comparator and equipment available has been very helpful in the ac-dc program, in both the research and calibration phase.

(12) Incorrect procedures, generally stemming from incomplete understanding, can also be major sources of error. As F. B. Silsbee has emphasized [14], the person in charge of a standardizing laboratory must have a high degree of technical knowledge and competence. This is especially true for ac-dc difference measurements, which are deceptively simple in principle but require a person with a sound knowledge of ac circuit theory to detect and overcome problems.

(13) It is to be expected that the ranges of the reference standards closest to those of the primary standards will be known with the best accuracy, and that the accuracy will decrease as both range and frequency are extended. Thus a realistic view of the uncertainty, $u$, will be that of a 
rather flat-topped cone in a 3-dimensional space, with the range and

frequency as $x$ and $y$ coordinates and $1 / u$ as the $z$ (vertical) coordinate. The views for the working standards will, of course, be similar.

\section{RANDOM (STATISTICALLY EVALUATED) ERRORS}

\section{A. MJTC Comparator}

A limiting factor in the precision of TE comparisons was the noise level of the circuit and nanovoltmeter. With the 10-s averager for each reading and with 120 s required for an ac-dc determination, the pass band of interest extended from about 0.01 to $0.1 \mathrm{~Hz}$. Both the noise level and the response time of the nVM were dependent on the source resistance. With these factors in mind, a number of tests were made to evaluate the effects of noise and zero drift of the comparator and its nVM on ac-dc measurements, with the two thermocouple inputs shorted.

The first of these consisted of eyeballing the peak-to-peak excursions of the nVM for $10 \mathrm{~s}$, while at the same time taking 10-s averages of the nVM output (expressed in nanovolts of input). The average difference between these 10-s averages was generally 1/8th to $1 / 20$ th of the average of the peak-to-peak excursions, a marked improvement. However a better measure of the effect of noise on the integrated output was obtained by calculating a phantom $\Delta^{\prime}=d_{T}-d_{S}$, by the formula given in section III, using groups of successive $10^{-s}$ readings and assuming $\mathrm{nE}=20 \mathrm{mV}$. For both the 300 and 1000-nV ranges of the nVM usually used, the average $\Delta^{\prime}$ (without regard to sign) was less than $0.1 \mathrm{ppm}$.

Similar (although fewer) measurements were made with maximum source resistance $(300 \Omega)$, and with $30 \mathrm{~s}$ between averages, as in a normal ac-dc comparison. The values of $\Delta^{\prime}$ ranged up to $0.15 \mathrm{ppm}$. They would be expected to be 3.3 times as great with emf's of $3 \mathrm{mV}$, as in the TVC build-ups. These 
are the true limiting minimums of random errors imposed by the comparison process.

\section{B. Other Comparators}

Similar tests were not made for the 1966 comparator and for the calibration console. Both have been in use for long periods of time. Instead the pooled standard deviations of each were evaluated under the conditions of the present investigation, for TE and TVC comparisons, as discussed in the next sections.

\section{Statistical Parameters and Tests}

Four independent determinations were normally made at each frequency in each comparison of the TE's, and the average, $\Delta_{a}$, and its standard deviation, $s_{a}$, were computed. Since the same measurement process was involved in many such tests, pooled standard deviations were computed to increase the precision of estimating the true $\sigma$. If each of $\mathrm{m}$ comparisons has the same number of determinations, the formula for the pooled standard deviation of the average is simply

$$
s_{p a}=\left(\frac{1}{m} \sum_{1}^{m} s_{a i}\right)^{1 / 2} .
$$

Preliminary calculations were needed to determine which tests to pool. Obviously comparisons at low emf's were likely to have larger $s_{a}$ 's than those at $10 \mathrm{mV}$. On the other hand tests at different frequencies rarely had significantly different $s_{a}$ 's.

The pooled "within-day" $s_{a}$ 's were used to more sharply determine the significance of differences between averages of measurements repeated on different days. For two measurements the "t" test, with the formula given in section III, was simplest. For more measurements, appropriate tests were used [15]. 
The round-robin triads of figures 3 and 4, the build-up comparisons for the TVC's, and the comparisons of ampere-range TE's with shunted TE's at low frequencies were all examined for evidence of systematic errors.

\section{Pooled Standard Deviations}

Table 14 shows the values of the pooled standard deviations, $s_{p a}$, for the several paths in the Road Map of figure 1. Each was grouped by parameters, such as type of comparator, frequency, and emf level, that were found to be significant.

The table shows the wide range of $s_{p a}$ 's from the MJTC's to the working standards. These values are combined in section XIII, with the systematic error estimates of section XII.

\section{E. Experimental Designs and Tests}

The sequence of readings in a determination (ac, dct, $d c^{-}, a c$, equally spaced in time) is an example of a simple experimental design that eliminates the error from linear drifts in the TE's and comparator. Knight and his coworkers at NPL have used a least-squares fit to the readings in multiple ac-dc sequences to correct for 2 nd and $3 r d$ order drifts as well. However the excellent temperature stability in MET A144 at NBS, and the long thermal time-constant afforded by the thermally-insulated tank in which the TE's are placed, have made this unnecessary for this investigation. Erratic changes, chiefly from the nVM, would generally mask small non-linearities.

Experimental designs based on left-right interchanges of two standards to detect offsets could not be used directly, because of the often large differences in the emf's of the TE's (as great as $3 / 1$ ). With the MJTC comparator, the higher emf must be connected to the divider, and the output of the TE with the higher $\mathrm{n}$ must be connected to the potentiometer. 
Triads of comparisons (round robins) are shown in figures 3 and 4 . In each of these there are two paths from one TE to a second; one direct and the other via the third TE. If the $d_{2}-d_{1}$ paths differ by more than $(2 \sqrt{3}) s_{p a}$, there is evidence of systematic error at about the $95 \%$ confidence level. None of these 28 triads showed such evidence. Other more complicated round robins, used to evaluate TE's after the loss of FX and FY, also showed small closure errors.

In the 50 to $5 \mathrm{~mA}$ comparisons of figure 3, the values of $d$ for $F Y$ and FZ, obtained after 4 stages from OF 19, were within 1 ppm of those obtained from the direct MJTC tests at $1 \mathrm{kHz}$ and the subsequent frequency extensions.

\section{ESTIMATES OF RESIDUAL SYSTEMATIC ERRORS (NOT STATISTICALLY EVALUATED)}

There are many known sources of residual errors in the TE's, TVC's and comparator. A listing of those which are significant and of the estimates of their limits (bounds) is given in ppm (and percent of $\Delta=d_{T}-d_{S}$ where applicable) in table 15 for each of the blocks in the Road Map of figure 1. The estimates of limits are given for $1 \mathrm{kHz}$, where many errors are at a minimum, and for the highest frequency. Unless otherwise noted, equal bounds are implied; i.e., 0.5 signifies that it is considered to be very unlikely that the residual error will be outside the range -0.5 to $+0.5 \mathrm{ppm}$.

Many of the bounds were determined from actual measurements or specifications. Others were difficult to estimate, and a few are simply educated guesses; based, however, on extensive experience in ac-dc measurements. Some pertinent notes are given at the end of the table. Almost all of the sources of error are independent (not correlated). The few correlated sources are discussed in the next section. 


\section{COMBINATION OF UNCERTAIMTIES}

From the basic dimensionless equation given in section III-A $d_{T}=d_{S}+\left(N_{d}-N_{a}\right) / n_{S} E_{l}$. Here the exponent of each variable is +1 or -1 . From the theory of errors [16], the propagation-of-error formula for the fractional standard deviation, $s_{r}$, of $d_{T}$, with uncorrelated variables having small random errors, reduces to $\left(\sum s_{i}^{2}\right)^{1 / 2}$, where each of the $s_{i}$ 's is the fractional standard deviation of an individual factor (considering $\left(N_{d}-N_{a}\right)$ as a factor). In accordance with Dietrich [12], the same formula will apply safely enough for combinations of estimated residual uncertainties ${ }^{5}$, each taken to be uniformly distributed between estimated bounds, $\pm b$, with the standard deviation of each taken as $s_{i}= \pm b_{i} / \sqrt{3}$. These values of $s_{i}$ are the "equivalent standard deviations" of the BIPM report (appendix 6). Twice the overall standard deviation, $2 s_{O}$, is taken as a reasonable measure of the overall uncertainty. According to Dietrich the probability of a value lying outside this will be less than 0.05 .

This use of $2 s_{0}$ for the overall uncertainty, $U_{O}$, is strongly recommended by Dunn of the National Research Council of Canada [17], who also recommends the approach taken by Dietrich to the combination of uncertainties. It seems more appropriate than $3 s_{0}$ when many of the sources of error are considered to be of uniform probability density, since the long tails of the normal distribution are truncated.

Because of the several successive steps and the three different comparators used in evaluating the NBS reference and working standards, and because of the many steps in the extensions of the frequency and other ranges, the rather straightforward procedure of combining uncertainties from tables 14 and 15 becomes very involved. The detailed calculations are shown in table 16. They are generally self-explanatory. The results are the same as those obtained by adding variances and finding the overall uncertainty as twice the square root of the overall variance. However, no definite confidence limit is associated with the uncertainty, $U$.

${ }^{5}$ with each other and with $s_{r}$. See section $x-4$. 
Extreme rigor in making these calculations did not seem warranted, especially for the smaller U's, which do not effect the rss results very much.

In the $F_{1}$ TVC build-up the errors in determining the changes in the $d_{c}$ 's of the TE's $\left(F X_{2}\right.$ and $\left.F Y_{2}\right)$ with current level are correlated; i.e., they add in each step of the build-up. Similarly in the $F_{7}$ TV build-up, the errors in determining the difference between the $d_{c}$ 's of $F_{7} 1$ and $F_{7}$ at the particular currents used add at each step. These correlated uncertainties were separately evaluated in the calculations of table 16.

It is to be noted that in each build-up involving chains of successive measurements the uncertainty at the end of each chain is given in table 16.

It is difficult to estimate the effects of the uncertainties which depend on the magnitude of $\Delta$, particularly for these chains of measurements. These range from $2.8 \%$ of A for the MJTC comparator to $3.6 \%$ for the other comparators. A prudent allowance for them is given in B of table 17 . Fortunately the values of $d$ for the NBS standards are small, so that this is a significant factor in only a few cases.

The values of $d$ and $U$ apply at the currents and voltages shown in the various tables. At other voltages and currents additional corrections must be applied to the d's, and the uncertainties will be somewhat greater. Appropriate currents and voltages were used in evaluating the reference standards. Fortunately the working standards are rarely used at other than rated inputs. 


\section{CONCLUSIONS}

\section{A. Uncertainties of the NBS Standards}

The overall results of the calculations of the uncertainties are given in the important summary table 17. They apply at rated current and voltage, with the exceptions shown.

Several conclusions can be drawn from this table and the other results of this study, as follows:

(1) The long-term stability of the d's of the MJTC's is excellent. This, together with their low d's and $0.5 \mathrm{ppm}$ uncertainty, amply fulfilled the hope that they would be superior primary standards of ac-dc difference at the base frequency of $1 \mathrm{kHz}$, and over the range from $30 \mathrm{~Hz}$ to $10 \mathrm{kHz}$.

(2) Their accuracy can be transferred to the single-junction TE's at $5 \mathrm{~mA}$ and 5 to $10 \mathrm{~V}$ to $1 \mathrm{ppm}$.

(3) The accuracy of these base ranges can be extended to $100 \mathrm{kHz}$ to better than 2 ppm.

(4) As expected, (see section $\mathrm{X}-13$ ) the uncertainty of the reference standards increases with increasing $V$ or $I$ range. The most precise comparator can only be used to $50 \mathrm{~mA}$ or $100 \mathrm{~V}$, so the uncertainty cone has discontinuities in slope.

(5) As expected, the difficulties of extending the range increase at higher frequencies, and so do the uncertainties; generally by a factor of 1.5 to 2 times those at $1 \mathrm{kHz}$.

(6) As shown in table 16, the random-error component is significant in almost all comparisons. There is generally a reasonable balance between 
random and systematic components. However, better precision would be the first step to improving accuracies.

\section{B. Calibration Uncertainties}

(1) If TE's and TVC's of the same type and quality as the working standards are calibrated with the calibration console, it would be expected that the overall uncertainty would be the rss of the working standards from table 17 and the single step U's of $16-\mathrm{H}$ (minus the $\mathrm{s}_{\mathrm{e}}$ of the reference standard) or 16-I of table 16. The results of such calculations are given in table 18, along with the present calibration allowances, from section I-C.

(2) As the table shows, little improvement may be possible for the coaxial TVC calibrations. For TCC calibrations the differences between the rss uncertainties and the allowances are deceptive. Individual TE's of the same quality as our standards are no longer avallable in the ampere ranges. The multirange TVC's with shunts that are commercially available are much more complex. Experience has indicated that they have larger and less stable d's than do the single-range TE's and coaxial TVC's.

(3) If NBS is pressed for better accuracy, particularly for TVC's, the needs might be met by using the uncertainty-cone concept to specify smaller uncertainties at and near the apex. In this way the basic accuracy at NBS could be transported via suitable ac-dc transfer standards to the client's standards laboratory.

(4) Very high accuracies are attainable over limited ranges for very special tests (such as international comparisons) in which such accuracies are really needed. They would be made by directly comparing single-range coaxial TVC's up to $100 \mathrm{~V}$ with the $\mathrm{F}_{1}$ resistors and reserve TE'S (FA and FB), or single-junction TE's up to $50 \mathrm{~mA}$ with one of the pairs of reference TE's, using the MJTC comparator in each case. The results of calculations (similar to those described in $B-1$ of this section) are shown in table 19, to substantiate this. 
(5) A comparison was made of the d's of the working standards shown in tables 11 and 13 with the values previously assigned from earlier tests and presently used in calibration work. For the $F_{7}$ TVC's, the largest difference between the d's was $23 \mathrm{ppm}$, on the 600-V range at $100 \mathrm{kHz}$ (the highest voltage available at this frequency). It was $10 \mathrm{ppm}$ on the $1000-\mathrm{V}$ range at $50 \mathrm{kHz}$, and much less at most other ranges and frequencies. The average difference for all ranges at all frequencies (with regard to sign) was only $2 \mathrm{ppm}$. For the current-measuring TE's the largest difference between the d's was $36 \mathrm{ppm}$. The average for all ranges at all frequencies was only $8 \mathrm{ppm}$. These low differences attest to both the quality of the earlier measurements and the stability of the NBS ac-dc transfer standards. They are comfortably below the uncertainties of calibrations shown in section I-C of this report.

\section{RECOMMENDATIONS}

\section{A. Maintenance of AC-DC Standards}

(1) The NBS standards are believed, with reason, to be very stable. However if better accuracy than heretofore is to be maintained, it would be prudent to compare the working standard TE's with the reference standards every three years, and to compare adjacent ranges of the $F_{7}$ TVC set at similar intervals. This could be modified by experience -- see recommendation $\mathrm{B}-6$.

(2) The difficult and very time-consuming recalibrations of the reference standards might only be necessary every 9 years, if the 3-year tests show no significant changes.

(3) Spare $\mathrm{F}_{7} 1$ and $\mathrm{F}_{7}$ modules should be available in case of burnout, with values $d_{v}$ and $d_{c}$ known by prior comparison with the present ones. FA and FB, an extra pair of 5-mA TE's with known $d_{v}$ and $d_{C}$, are already available for the $F_{1}$ reference set. The substitution formulas of section IX 
should make it possible to calculate the $d_{v}$ 's for the other TVC ranges, without recalibrating each one, when a TE is replaced.

(4) AC-DC differences as large as $9 \mathrm{ppm}$ have been observed even in Evanohm-heater TE's, so replacements should never be used without recalibration.

\section{B. Quality Control of the Calibration Process}

(1) The principles of quality control given by Eisenhart [13] should be followed. Check standards should be evaluated periodically in the calibration console. They should be of the same types as the major $k$ inds of ac-dc standards normally calibrated. In particular the multirange standard, which was purchased in part for this purpose, should be put in service, as well as the spare Model F TVC's on which a partial history has already been garnered.

(2) NBS statisticans should be consulted and should be of major help in setting up these programs. Careful selection of a few ranges and frequencies should not make these very important quality-control tests too time-consuming or onerous.

(3) The waveform and frequency of the applied ac should be observed on a calibrated CRO in each test, and the frequency setting of the oscillator should be double-checked to avoid mistakes.

(4) The very simple EMI test described in section III-A should be made occasionally, especially for low ranges.

(5) A second comparator and associated equipment should always be available and ready for use as a check against unsuspected (or suspected) errors. This is particularly valuable when new or modified ac-dc standards are first tested. 
(6) As experience with the check standards and the bienniel calibrations of the working standards is accumulated, the recommended callbration periods of $\mathrm{A}^{-1}$ for the working and reference standards may safety be revised.

\section{Other Recommendations}

(1) Almost all of the TE's from $5 \mathrm{~mA}$ to $20 \mathrm{~A}$ were made to our special order by American manufacturers who no longer make TE's. The MJTC's are not commercially available at all. All of these now form an unique collection of well-evaluated reference standards, and should be reserved solely for evaluating the NBS working standards and for comparisons with other national laboratories, or for other special test in which the highest accuracy is really required. The MJTC comparator, with its high precision, should be available for use when this is really needed.

(2) The new types of solid-state thermoelements (SSTE's) have much higher emf's than the conventional TE's, but are subject to the same basic limitations on ac-dc difference from Thomson, Peltier and other effects. If Wilkins'-type MJTC's can not be obtained commercially in the near future, serious consideration should be given to designing and making thin-film MJTC's by cooperation with other divisions at NBS.

(3) The new values of d for the working standards, from tables 11 and 13, should be used in place of those now in the computer. However any stated improvement in the calibration uncertainties should be withheld until some checks on the error analyses are made by comparing the NBS working and reference standard TE's and TVC's in both the calibration and 1966 consoles. In addition any improvement in the stated uncertainty of the more complex multirange TVC's and their shunts should await the installation of a quality-control program and further studies of these instruments. 


\section{ACKNOULEDGEMENTS}

Sincere thanks are expressed to J. R. Hastings, E. S. Williams, C. B. Childers, and K. B. Krishnamurthy, who assisted in this investigation, to $N$. Belecki for $h$ is encouragement and support, to $C$. Reeve for his helpful suggestions and review of the statistical procedures, and to $T$. Lipe for his review of the entire report. 


\section{REFERENCES}

1. Thermal Converters as AC-DC Transfer Standards for Current \& Voltage Measurements at Audio Frequencies, F. L. Hermach, Jour. Res. NBS, v. 28, no. 2, p. 121, Feb. 1952.

2. Thermal Converters for Audio-Frequency Voltage Measurements of High Accuracy, F. L. Hermach and E. S. Williams, IEEE Trans. on Instrum. \& Measmt., v. IM-15, p. 260, Dec. 1966.

3. Thermal Voltage Converters \& Comparator for Very Accurate AC Voltage Measurements, E. S. Williams, Jour. Res. NBS, v. 75C, p. 145, July-Dec. 1971 .

4. An Investigation of Multijunction Thermal Converters, F. L. Hermach and D. R. Flach, IEEE Trans. on Instrum. \& Measmt., v. IM-25, p. 524, Dec. 1976.

5. Thermal Voltage Converters for Accurate Voltage Measurements to 30 Megacycles per Second, F. L. Hermach and E. S. Williams, AIEE Trans., V. 79-I, p. 200, July 1960.

6. Thermal Current Converters for Accurate AC Current Measurement, E. S. Williams, IEEE Trans. on Instrum. \& Measmt., v. IM-25, p. 519, Dec. 1976.

7. Errors in AC-DC Transfer Arising from a DC Reversal Difference, B. D. Inglis, Metrologia, v. 17, p. 111, 1981.

8. Theoretical Analysis of the AC/DC Transfer Difference of the NPL Multijunction Thermal Converter Over the Frequency Range DC to $100 \mathrm{kHz}$, F. J. Wilkins, IEEE Trans. on Instrum. \& Measmt., v. IM-21, p. 334, Nov. 1972. 
9. Evaluation of AC-DC Transfer Errors for Thermal Converter-Multiplier Combinations, B. D. Inglis, Metrologia, v. 16, p. 177, 1980.

10. Uncertainties in Calibrations, W. Youden, IRE Trans. Instrum., v. I-11, p. 133, Dec. 1962.

11. Uncertainty Assignment, B. Taylor, Memo for Technical Staff of the NBS Electriclty Division, Aug. 21, 1981.

12. Uncertainty, Calibration, and Probability, C. F. Dietrich, Halsted Press, 1973.

13. Realistic Evaluation of the Precision and Accuracy of Instrument Calibration Systems, C. Eisenhart, J. Res. NBS, v. 67C, p. 161, Apr-June 1963.

14. Suggested Practlces for Electrlcal Standardizing Laboratorles, F. B. Silsbee, NBS C1rcular 578, 1956.

15. Experimental Statist1cs, M. G. Natrella, NBS Handbook 91, 1966 (Chapters 3 and 15).

16. Notes on the Use of Propagation of Error Formulas, H. H. Ku, J. Res. NBS, v. 70C, p. 263, Oct-Dec 1966.

17. Measurement Assurance, A. F. Dunn, NRC, Canada, Report 18843, 1980.

18. A Resistor Calculable from $A C$ to $w=10^{5} \mathrm{rad} / \mathrm{s}, \mathrm{R}$. J. Haddad, Thesis, GWU School of Engineering and Applied Science, April 1969.

19. The Theory of Peltier \& Thomson Errors in Thermal AC-DC Transfer Devices, F. C. W1ddis, IEE Monograph 497M, Jan 1962. 
Table 1

Comparisons of Four MJTC's

\begin{tabular}{|c|c|c|c|c|c|c|}
\hline \multirow[b]{2}{*}{ v } & \multirow[b]{2}{*}{$\mathrm{T}$} & \multirow[b]{2}{*}{$s$} & \multicolumn{3}{|c|}{$-d_{T}-d_{S}(p p m)$} & \multirow[b]{2}{*}{ Date } \\
\hline & & & $30 \mathrm{~Hz}$ & $1 \mathrm{kHz}$ & $10 \mathrm{kHz}$ & \\
\hline \multirow[t]{2}{*}{10.0} & $\begin{array}{c}\text { G44 } \\
\text { (B10-44) }\end{array}$ & $\begin{array}{c}G 5 C \\
(B 5-2)\end{array}$ & +0.47 & +0.15 & -0.44 & \\
\hline & & & & -0.10 & & $12 / 11 / 81$ \\
\hline 6.0 & & & & -0.02 & & $12 / 11 / 81$ \\
\hline \multirow[t]{2}{*}{6.0} & $\begin{array}{l}\text { Gr } 25-1 \\
(\text { C } 10-1)\end{array}$ & $\begin{array}{c}G 5 C \\
(B 5-2)\end{array}$ & +0.32 & & -0.29 & \\
\hline & & & & -0.03 & & $12 / 14 / 81$ \\
\hline \multirow[t]{2}{*}{6.0} & $\begin{array}{l}\text { Gr25-1 } \\
(\mathrm{C} 10-1)\end{array}$ & $\begin{array}{c}\text { G44 } \\
(B 10-44)\end{array}$ & +0.17 & -0.01 & -0.05 & $12 / 14 / 81$ \\
\hline & & & & +0.06 & & $12 / 18 / 81$ \\
\hline \multirow[t]{3}{*}{8.0} & $\begin{array}{c}\text { NPL } 14 \\
(A 50-14)\end{array}$ & $\begin{array}{c}G 44 \\
(B 10-44)\end{array}$ & & +0.34 & & $12 / 18 / 81$ \\
\hline & & & & +0.36 & & $12 / 18 / 81$ \\
\hline & & & -0.40 & +0.27 & & $12 / 18 / 81$ \\
\hline 6.0 & $\begin{array}{l}\text { Gr } 25-1 \\
\text { (C10-1) }\end{array}$ & $\begin{array}{c}\text { NPL } 14 \\
(A 50-14)\end{array}$ & +0.52 & -0.22 & & $12 / 18 / 81$ \\
\hline
\end{tabular}


Table 2

Differences Between 1981 and 1976 Evaluations of MJTC's

\begin{tabular}{cccc} 
& \multicolumn{3}{c}{$\mathrm{d}_{81}-\mathrm{d}_{76}(\mathrm{ppm})$} \\
\cline { 2 - 4 } MJTC & $\underline{30 \mathrm{~Hz}}$ & $\underline{1 \mathrm{kHz}}$ & $\frac{10 \mathrm{kHz}}{0.0}$ \\
Gr25-1 & +0.2 & 0.0 & +0.1 \\
G5C & 0.0 & 0.0 & -0.4 \\
G10C & +0.3 & -0.1 & +0.1 \\
NPL 14 & -0.3 & 0.0 & 0.0
\end{tabular}


Table 3

Results of Comparisons of FX and FY with MJTC's

\begin{tabular}{|c|c|c|c|c|}
\hline \multirow{2}{*}{$\begin{array}{l}\text { TE } \\
\text { or } \\
\text { TVC } \\
\end{array}$} & \multirow{2}{*}{$\begin{array}{r}I \\
\text { or } \\
\mathbf{V}\end{array}$} & \multicolumn{3}{|c|}{$d(p p m)$} \\
\hline & & $30 \mathrm{~Hz}$ & $1 \mathrm{kHz}$ & $10 \mathrm{kHz}$ \\
\hline $\mathrm{FX}$ & $5 \mathrm{~mA}$ & - & -0.3 & -0.2 \\
\hline $\mathrm{FX}$ & $2.5 \mathrm{~mA}$ & - & 0.0 & -0.1 \\
\hline $\mathrm{F}_{15}-\mathrm{FX}^{1}$ & $5 \mathrm{~V}$ & +0.9 & -0.4 & -1.2 \\
\hline $\mathrm{F}_{15-\mathrm{FX}}$ & $3 \mathrm{~V}$ & -0.1 & -0.7 & -1.0 \\
\hline FY & $5 \mathrm{~mA}$ & - & +0.2 & 0.0 \\
\hline FY & $3 \mathrm{~mA}$ & - & +0.4 & -0.2 \\
\hline $\mathrm{F}_{1} 10-\mathrm{FY}$ & $10 \mathrm{~V}$ & +0.8 & -0.3 & -0.8 \\
\hline $\mathrm{F}_{1} 10-\mathrm{FY}$ & $5 \mathrm{~V}$ & +0.2 & +0.1 & -1.2 \\
\hline
\end{tabular}

${ }^{1} \mathrm{~F}_{15}-\mathrm{FX}$ consists of the $5-\mathrm{V}$ resistor of the $\mathrm{F}_{1}$ TVC set, in series with TE FX. 
Table 4

Results of TVC Tests with Twin Resistors

\begin{tabular}{|c|c|c|c|c|c|}
\hline \multirow[b]{2}{*}{$\mathrm{T}$} & \multirow[b]{2}{*}{$s$} & \multicolumn{4}{|c|}{$d_{T}-d_{S}(p p m)$} \\
\hline & & $30 \mathrm{~Hz}$ & $1 \mathrm{kHz}$ & $20 \mathrm{kHz}$ & $50 \mathrm{kHz}$ \\
\hline FY & $F X$ & +0.2 & +0.2 & 0.0 & -0.2 \\
\hline B5E77 & FX & +5.0 & +2.6 & +3.2 & +3.7 \\
\hline F5K9 & $F X$ & -1.6 & -1.0 & -1.1 & -1.2 \\
\hline B5EXG1 & $F X$ & +2.8 & +1.1 & +0.9 & +1.5 \\
\hline A5EP7 & $F X$ & -0.1 & +0.5 & -0.5 & $-1 \cdot 3$ \\
\hline $\begin{array}{l}d \text { of } \\
d_{F X}=\end{array}$ & & & +0.6 & +0.4 & +0.4 \\
\hline
\end{tabular}

NOTE: All tests at $5 \mathrm{~mA}$.

$30 \mathrm{~Hz}$ results not averaged. See text. 
Table 5

Ampere-Range Comparisons

$\mathbf{d}_{\mathbf{T}}-\mathbf{d}_{\mathbf{S}}$

I

(A)

$20 \mathrm{~Hz}$

$1 \mathrm{kHz}$

$20 \mathrm{kHz}$

$50 \mathrm{kHz}$

\begin{tabular}{|c|c|c|c|c|c|c|c|}
\hline W20A & W10A & $\# 0-1$ & 10 & +1 & +11 & +17 & +10 \\
\hline W1OA $0-1$ & W5A & $\# 5-2$ & 5 & -5 & -2 & 0 & +16 \\
\hline W1OA 非0-1 & W5A & \#5-1 & 5 & -6 & -6 & -6 & +17 \\
\hline W5A 非 $5-2$ & W3A & $\# 4-1$ & 3 & +1 & -1 & +4 & +16 \\
\hline W3A \# 4-1 & W2A & 非3-2 & 2 & +3 & 0 & -7 & -11 \\
\hline W2A 非3-2 & W1A & $\# 7-2$ & 1 & -4 & -2 & -5 & -13 \\
\hline FG3A & W3A & $\# 4-1$ & 3 & -2 & 0 & +15 & +43 \\
\hline FG3A & W3A & \#4-1 & 2 & -4 & +1 & +15 & +46 \\
\hline FG1A & W1A & $\# 7-2$ & 1 & -3 & -2 & +11 & +29 \\
\hline FG1A & W1A & \#1-2 & 0.6 & +1 & 0 & +15 & +36 \\
\hline $\mathrm{FH} 2 \mathrm{~A}$ & W2A & $\# 3-2$ & 1.2 & -2 & -2 & -12 & -40 \\
\hline $\mathrm{FH} 2 \mathrm{~A}$ & W2A & \#3-2 & 2.0 & & 0 & -14 & -33 \\
\hline $\mathrm{FH} 2 \mathrm{OA}$ & W20A & $\# 7-1$ & 10 & & & -21 & -49 \\
\hline $\mathrm{FH} 20 \mathrm{~A}$ & W20A & $\# 7-1$ & 16 & & & -22 & -59 \\
\hline
\end{tabular}

NOTE: FG1A, FH2A, FG3A, and FH2OA are Williams' shunts with low-range TE's, $\mathrm{FG}$ and $\mathrm{FH}$. 


\section{Table 6}

AC-DC Differences of $1 \mathrm{~A}$ to $20 \mathrm{~A} \mathrm{TE} ' \mathrm{~s}$

\begin{tabular}{|c|c|c|c|c|c|c|}
\hline \multirow[b]{2}{*}{ TE } & & \multirow{2}{*}{$\begin{array}{c}I \\
(A)\end{array}$} & \multicolumn{4}{|c|}{$d(p p m)$} \\
\hline & & & $20 \mathrm{~Hz}$ & $1 \mathrm{kHz}$ & $20 \mathrm{kHz}$ & $50 \mathrm{kHz}$ \\
\hline W2OA & $\# 7-1$ & 10 & -5 & +5 & +14 & +10 \\
\hline W10A & $\# 0-1$ & 5 & -6 & -6 & -3 & 0 \\
\hline W5A & 非 $5-2$ & 3 & -1 & -4 & -3 & -16 \\
\hline W3A & 非 4-1 & 2 & -2 & -3 & -7 & -32 \\
\hline W2A & $\# 3-2$ & 1 & -5 & -3 & 0 & -21 \\
\hline W1 A & \#1 7-2 & 1 & -1 & -1 & +5 & -8 \\
\hline W1 A & $\# 2-2$ & 1 & -4 & -3 & +2 & -10 \\
\hline
\end{tabular}


Table 7

Results of Evaluation of $\mathrm{FX}_{2}$ and $\mathrm{FY}_{2}$

\begin{tabular}{|c|c|c|c|c|c|c|c|}
\hline \multirow{3}{*}{$\begin{array}{r}\text { TE } \\
\text { or } \\
\text { TVC }\end{array}$} & \multirow{3}{*}{$\begin{array}{r}I \\
\text { or } \\
\text { V } \\
\end{array}$} & \multicolumn{6}{|c|}{$d(p p m)$} \\
\hline & & & & & & & \\
\hline & & $20 \mathrm{~Hz}$ & $1 \mathrm{kHz}$ & $10 \mathrm{kHz}$ & $20 \mathrm{kHz}$ & $50 \mathrm{kHz}$ & $100 \mathrm{kHz}$ \\
\hline \multirow[t]{3}{*}{$\mathrm{F}_{1} 10-\mathrm{FY}_{2}$} & $10 \mathrm{~V}$ & +3.9 & +2.4 & $+1 \cdot 3$ & +1.0 & +0.6 & +0.3 \\
\hline & 7 & +2.0 & +1.4 & +0.6 & & & \\
\hline & 5 & +1.6 & \pm 1.4 & 0.0 & & & \\
\hline \multirow[t]{3}{*}{$\mathrm{FY}_{2}$} & $5 \mathrm{~mA}$ & +4.7 & +3.2 & & +3.6 & +3.5 & +3.9 \\
\hline & 3.3 & +2.8 & +2.4 & & & & +2.9 \\
\hline & 2.5 & +2.2 & +2.3 & & & & \\
\hline \multirow[t]{3}{*}{$\mathrm{FX}_{2}$} & $5 \mathrm{~mA}$ & +3.5 & +2.2 & & +2.4 & +2.1 & +2.2 \\
\hline & 3.3 & +2.3 & +1.3 & & & & $+1 \cdot 3$ \\
\hline & 2.5 & +1.6 & +1.9 & & & & \\
\hline
\end{tabular}


Table 8

Comparisons of FitVC's

$\left(F X_{2}\right.$ and $F Y_{2} T E^{\prime} s$ )

$\Delta=\mathrm{d}_{\mathrm{T}}-\mathrm{d}_{\mathrm{S}}(\mathrm{ppm})$

T

$S$ S

v

$\mathrm{F} 200-\mathrm{FX}_{2} \quad \mathrm{~F} 100-\mathrm{FY}_{2}$

100

50

$\mathrm{F} 100-\mathrm{FY}_{2} \quad \mathrm{~F}^{2} \mathrm{O}-\mathrm{FX}_{2}$

30

20

10

$\mathrm{F}^{20}-\mathrm{FX}_{2} \quad \mathrm{~F} 10-\mathrm{FY}_{2}$

$\mathrm{F} 10-\mathrm{FY}_{2} \quad \mathrm{~F}_{5}-\mathrm{FX}_{2}$

$\mathrm{F}_{5}-\mathrm{FX}_{2} \quad \mathrm{~F}_{3}-\mathrm{FY}_{2}$

$\mathrm{F}_{3}-\mathrm{FY}_{2} \quad \mathrm{~F} 2-\mathrm{FX}_{2}$

$\mathrm{F}_{2}-\mathrm{FX}_{2} \quad \mathrm{~F} 1-\mathrm{FY}_{2}$

$\mathrm{F} 1-\mathrm{FY}_{2} \quad \mathrm{FX}_{2}$

$\mathrm{FX}_{2} \quad \mathrm{FY}_{2}$

0.5

$-1.1$

$+0.2$

$-0.8$

$-1.6$

$-0.3$

0.0

0.0

$+1.4$

$+0.4$

$+1.9$

$+1.0$

$+3.4$

$+1.1$
$-2.9$

$-1 \cdot 3$

$-3.1$

$-6.3$

$-2.1$

$-0.3$

$-0.8$

$+0.7$

$-1.1$

$+0.7$

$-3.9$

$+4.2$

$+1.9$

$+2.2$

$+2.5$

Comparisons with $F_{7}, F X$, and $F Y$ in place of $F X_{2}$ and $F Y_{2}$

$\begin{array}{llllllll}\text { F1000-F } 71 & \text { F500-FX } & 500 & - & -2.0 & -15.1 & -1.7 & - \\ \text { F500-FX } & \text { F300-FY } & 300 & - & -0.8 & -0.3 & -4.1 & -33 \\ \text { F300-FY } & \text { F200-FX } & 200 & - & -0.7 & -0.5 & -2.3 & +6.7\end{array}$


Table 9

AC-DC Differences of $F_{1}$ TVC's with $F X_{2}$ and $F Y_{2}$

$d(p p n)$

TVC

$\mathrm{F} 1000 \mathrm{E}-\mathrm{F}_{7} 1$

$\mathrm{F} 500-\mathrm{FX}_{2}$

$\mathrm{F} 300-\mathrm{FY}_{2}$

$\mathrm{F}^{2} \mathrm{O0}-\mathrm{FX}_{2}$

$\mathrm{F} 100-\mathrm{FY}_{2}$

$\mathrm{F} 50-\mathrm{FX}_{2}$

$\mathrm{F} 30-\mathrm{FY}_{2}$

$\mathrm{F}^{20}-\mathrm{FX}_{2}$

$\mathrm{F} 10-\mathrm{FY}_{2}$

$F 5-F_{2}$

F3-FY 2

$\mathrm{F} 2-\mathrm{FX}_{2}$

$\mathrm{F} 1-\mathrm{FY}_{2}$

$\mathrm{FX}_{2}$

$\mathrm{FY}_{2}$
500

500

300

300

200

200

100

100

50

50

30

30

20

20

10

10
5

5
3

3
2

2
1

1
0.5

0.6

0.6

$\begin{array}{ll}- & +2.7 \\ - & +2.5 \\ - & +1.7\end{array}$

$\begin{array}{ll}- & +3.5 \\ - & +2.7\end{array}$

$+2.7$

$-\quad+2.4$

$+1.6$

$\begin{array}{ll}- & +2.7 \\ - & +1.9\end{array}$

- $\quad+1.3$

-
$-\quad+0.5$

$+2.6+3.0$

$+0.7+2.2$

$+2.0$

$+1.5$

$+0.7$

$+3.9$

$+1.4$

+2.4
+1.6

$\begin{array}{ll}+2.0 & +1.4 \\ +0.7 & +0.6\end{array}$

+2.3
+0.4

+1.2
+0.4

$+0.4$

$-1.5$

-1.0
-1.8

$-1.9$

$-4.4$

$-3.7$

$-4.5$

$-5.4$

$-7.9$

$-6.6$

$-8.9$
$20 \mathrm{kHz}$

$-11.5$

$-6.1$

$+1.2 \quad-6.5$

$+0.4-7.3$

$-18.6$

$-19.4$

$+1.9 \quad-1.8$

$+1.1$

$-2.6$

$+15.6$

$+14.8$

$$
\begin{array}{lll}
+0.4 & -1.7 & +6.4 \\
-0.4 & -2.5 & +5.6
\end{array}
$$

$\begin{array}{lll}-0.6 & -3.9 & -12.2 \\ -1.4 & -4.7 & -13.0\end{array}$

$$
\begin{array}{lll}
-0.6 & -1.9 & -6.8 \\
-1.4 & -2.7 & -7.6
\end{array}
$$

$+0.2 \quad-0.7$

$-0.6-1.5$

$-6.1$

$-6.9$

$-0.3$

$-1.1$

$-1.5$

$-2.3$

$-4.9$

$-5.7$

$+0.3$

$+1.0$

$+0.2$

$+0.6$

$-0.2$

$-0.5$

$+0.5$

$-0.3$

$+1$.

$+0.3$

$+2.0$

$+1.2$

$+0$.

+0.5
-0.3

$+3.4$

$+7.5$

$+6.7$

$-1.0$

$-1.3$

$+3.7$

$+3.9$

$+10.8$

$+10.0$

$-2.5$

$-3.3$

$+6.8$

$+6.0$

$+23.1$

$+22.3$

$-7.5$

+6 .

$+28.4$

$-9$.

$+4$.

$+25.9$ 
Table 10

Comparisons of Working and Reference Standard TE's

\begin{tabular}{|c|c|c|c|c|c|c|}
\hline \multirow[b]{2}{*}{ Test } & \multirow[b]{2}{*}{ Standard } & \multirow{2}{*}{$\begin{array}{c}I \\
(\mathrm{~mA})\end{array}$} & \multicolumn{4}{|c|}{$\Delta(\mathrm{ppm})$} \\
\hline & & & $20 \mathrm{~Hz}$ & $1 \mathrm{kHz}$ & $20 \mathrm{kHz}$ & $50 \mathrm{kHz}$ \\
\hline A3 \#95 & FA & 5 & +5 & +2 & +9 & +18 \\
\hline G10 非57 & B10EP2 & 10 & -5 & -5 & -4 & -3 \\
\hline F20 \#1 & W25E1 & 20 & -9 & -7 & -5 & +1 \\
\hline W30 非 1 & W25E1 & 25 & -12 & -11 & -12 & -12 \\
\hline F50 非2 & W50E1 & 50 & -37 & 0 & +3 & -2 \\
\hline B100 非3 & W100E1 & 100 & -18 & -20 & -20 & -24 \\
\hline A250 非 4 & W250E1 & 250 & -19 & -22 & -28 & -44 \\
\hline & & (A) & & & & \\
\hline wo. $5 \mathrm{~A} \quad \# 0-2$ & Wo. $5 \mathrm{~A}$ 非 $0-1$ & 0.5 & -4 & -2 & -5 & -2 \\
\hline W1A 非7-1 & W1A 非2-2 & 1 & 0 & -2 & -1 & -4 \\
\hline W2A \#8-3 & W2A 非3-2 & 2 & +3 & +1 & +1 & -2 \\
\hline W3A \# & W3A \#4-1 & 3 & +2 & -1 & -1 & -3 \\
\hline W5A 非-1 & W5A 非 $5-2$ & 5 & -5 & -7 & -11 & -13 \\
\hline
\end{tabular}

$\underline{25 \mathrm{~Hz}}$

W10A $\# 0-3 \quad$ W1OA $\# 0-1 \quad 10$

$-6$

$-5 \quad-13$

$-28$

$100 \mathrm{~Hz}$

W20A 非-2 W2OA 非-1

16

$+3$

$-10$

$-18$

$-14$ 
Table 11

AC-DC Differences of Working Standard TE's

\begin{tabular}{|c|c|c|c|c|c|}
\hline \multirow[b]{2}{*}{ TE } & \multirow{2}{*}{$\underset{(\mathrm{mA})}{I}$} & \multicolumn{4}{|c|}{ AC-DC Difference (ppm) } \\
\hline & & $20 \mathrm{~Hz}$ & $1 \mathrm{kHz}$ & $20 \mathrm{kHz}$ & $50 \mathrm{kHz}$ \\
\hline A5 非95 & 5 & +2 & 0 & +8 & +17 \\
\hline G10 非57 & 10 & -6 & -6 & -5 & -4 \\
\hline F20 非 1 & 20 & -10 & -8 & -6 & 0 \\
\hline W30 非 1 & 30 & -13 & -12 & -13 & -13 \\
\hline F50 非2 & 50 & -36 & -1 & +2 & -3 \\
\hline B100 \#3 & 100 & -20 & -21 & -20 & -23 \\
\hline A250 \# 4 & 250 & -24 & -22 & -28 & -43 \\
\hline
\end{tabular}

(A)

W0. $5 \mathrm{~A}$ 非-2

0.5

$-2$

$-3 \quad-7$

W1A 非7-1

1

$-5$

$+1$

$-14$

W2A 非-3

2

$-2$

$+1$

$-23$

W3A \#9-2

3

$-4$

$-8$

$-35$

W5A \#5-1

5

$-6$

$-11$

$-14$

$-29$

\section{$25 \mathrm{~Hz}$}

W1 OA 非0-3

10

$-12$

$-11$

$-16$

$-28$

\section{$100 \mathrm{~Hz}$}

W20A 非1-2

16

$+8$

$-5$

$-4$

$-4$ 
Table 12

Comparisons of $F_{7}$ TVC's

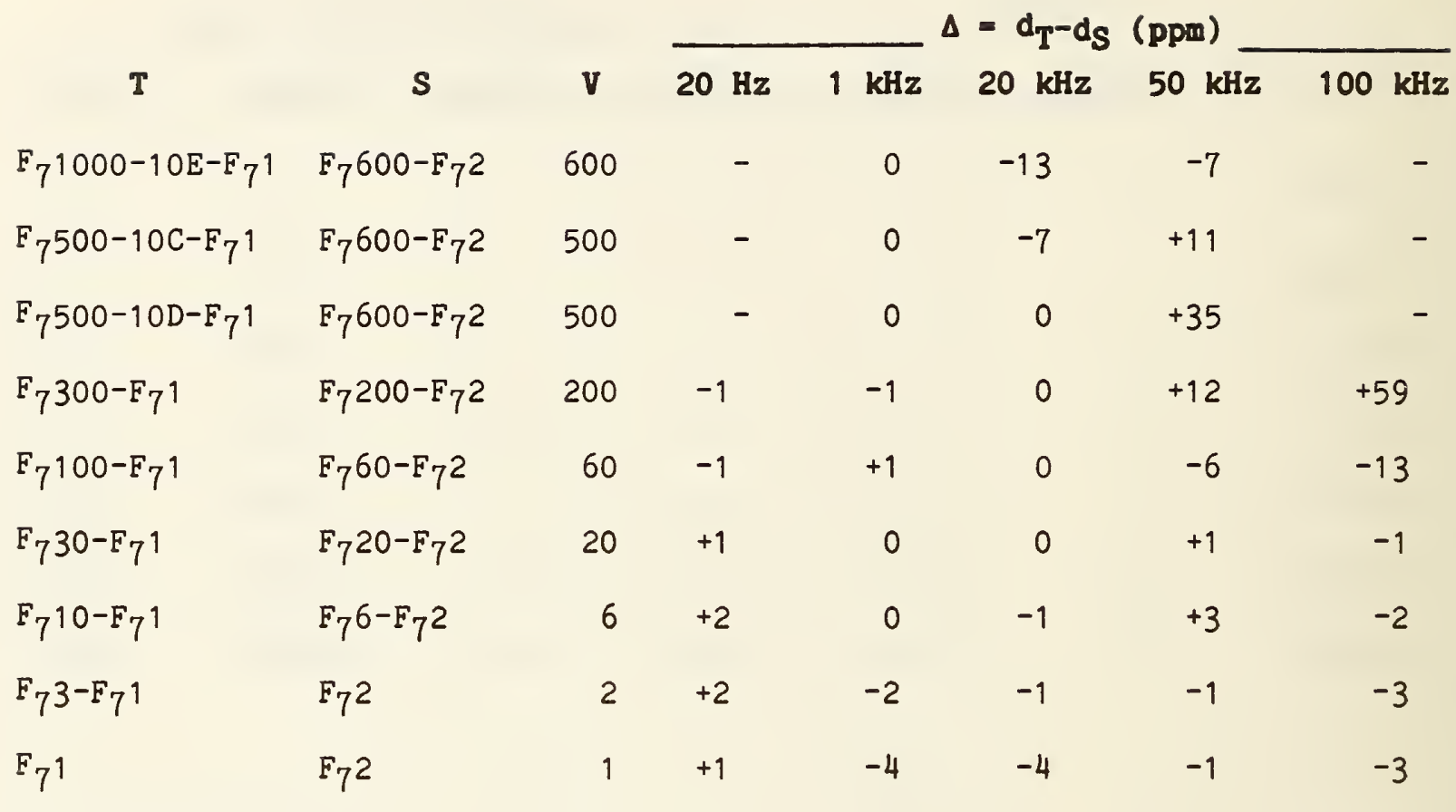


Table 13

\section{AC-DC Differences of $\boldsymbol{F}_{\boldsymbol{7}}$ TVC's}

$d(p p m)$

\begin{tabular}{|c|c|c|c|c|c|c|c|}
\hline TVC & $\mathbf{v}$ & $20 \mathrm{~Hz}$ & $1 \mathrm{kHz}$ & $20 \mathrm{kHz}$ & $50 \mathrm{kHz}$ & 100 & $\mathrm{kHz}$ \\
\hline $\mathrm{E}_{7} 1000-10 \mathrm{E}-\mathrm{E}_{7} 1$ & 600 & - & -2 & -15 & -2 & & - \\
\hline $\mathrm{E}_{7} 500-10 \mathrm{C}-\mathrm{F}_{7} 1$ & 500 & - & -2 & -9 & +16 & & - \\
\hline $\mathrm{F}_{7} 500-10 \mathrm{D}-\mathrm{F}_{7} 1$ & 500 & - & -2 & -2 & +40 & & - \\
\hline $\mathrm{E}_{7} 600-\mathrm{E}_{7} 2$ & 500 & -4 & -2 & -2 & +5 & & +43 \\
\hline $\mathrm{E}_{7} 300-\mathrm{F}_{7} 1$ & 300 & -4 & -2 & -2 & +5 & & +43 \\
\hline $\mathrm{E}_{7} 200-\mathrm{F}_{7} 2$ & 200 & -3 & -1 & -2 & -7 & & -16 \\
\hline $\mathrm{E}_{7} 100-\mathrm{F}_{7}{ }^{1}$ & 100 & -3 & -1 & -2 & -7 & & -16 \\
\hline $\mathrm{E}_{7} 60-\mathrm{E}_{7} 2$ & 60 & -2 & -2 & -2 & -1 & & -3 \\
\hline $\mathrm{F}_{7} 30-\mathrm{F}_{7} 1$ & 30 & -2 & -2 & -2 & -1 & & -3 \\
\hline $\mathrm{E}_{7} 20-\mathrm{F}_{7}{ }^{2}$ & 20 & -3 & -2 & -2 & -2 & & -2 \\
\hline $\mathrm{F}_{7} 10-\mathrm{F}_{7}$ & 10 & -3 & -2 & -2 & -2 & & -2 \\
\hline $\mathrm{F}_{7} 6-\mathrm{F}_{7} 2$ & 6 & -5 & -2 & -1 & -5 & & 0 \\
\hline $\mathrm{E}_{7}{ }^{3-\mathrm{F}_{7}}$ & 3 & -5 & -2 & -1 & -5 & & 0 \\
\hline $\mathrm{E}_{7}{ }^{2}$ & 2 & -7 & 0 & 0 & -4 & & +3 \\
\hline$E_{7} 1$ & 1 & -6 & -4 & -4 & -5 & & 0 \\
\hline
\end{tabular}


Table 14

Pooled Standard Deviations, $S_{p a}$

$S_{p a}$ (ppm of input $V$ or I)

Typical

emf (mV) $20 \mathrm{~Hz} 1 \mathrm{kHz} 20 \mathrm{kHz} 50 \mathrm{kHz} 100 \mathrm{kHz}$ Average

1. Comparison of

$10-40$

0.110 .16

0.14

MJTC's

(5) (11)

2. Comparison of

10

0.20

0.29

(5)

(9)

Reference TE's

and $\mathrm{F}_{1} 10 \mathrm{FY}_{2}$

3. Extension of

of frequency

range

0.23

0.24

0.25

0.24

4. Build up-

Reference TE's

a. 5 to $50 \mathrm{~mA}$

0.23

0.35

0.26

0.28

b. $50 \mathrm{~mA}$ to $1 \mathrm{~A}$

10

(11)

(13)

(11)

(11)

$1.80 \quad 1.68$

1.57

1.98

1.76

(11)

(16)

(15)

(17)

c. $1 \mathrm{~A}$ to $20 \mathrm{~A}$

5

$1.77 \quad 1.56$

1.48

1.74

(13)

(12)

(12)

(16)

5. Build up-

$\mathrm{F}_{1}$ TVC's
a. 0.5 to $100 \mathrm{~V}$
3
$0.49 \quad 0.47$
0.52
0.57
0.54
0.52
(7)
(12)
(12)
(10)
(10)
b. 200 to $1000 \mathrm{~V}$
$-1.71$
1.41
1.06
1.55
1.43
(4)
(4)
(3)
(2)

6. Comparison of Reference and 2.36

$$
2.77
$$

Working TE's

$5 \mathrm{~mA}-20 \mathrm{~A}$

7. Build up

$\mathrm{F}_{7}$ TVC's

1 to $1000 \mathrm{~V}$

3

$1.58 \quad 1.20$

2.16

2.54

2.79

2.05

(6)

(9)

(9)

(9)

(9)

${ }^{1}$ Values in parentheses are the number of tests. 


\section{Table 15}

\section{Estimated Limits of Residual Systenatic Uncertainties \\ (not evaluated by statistical means) \\ (See notes at end of table)}

\section{Limits, $\pm b$}

$\frac{1 \mathrm{kHz}}{\text { ppm \& of } \Delta} \frac{100 \mathrm{kHz}}{\mathrm{ppm} \text { \& of } \Delta}$

A. MJTC Comparator \& Equipment

1. $n V m$ \& averager (2\% of indication)

2. Determination of $n_{s}$

3. n-Compensation

4. DC-reversal difference definitions

5. AC effects

$$
b_{0}=\left(\Sigma b^{2}\right)^{1 / 2}
$$

$\begin{array}{cccc}- & 2 & - & 2 \\ - & 2 & - & 2 \\ 0.1 & & 0.1 & \\ 0.1 & & 0.1 & \\ 0.1 & & \underline{0.2} & - \\ 0.17 & 2.8 & 0.25 & 2.8\end{array}$

B. 1966 Comparator \& Equipment

1. 1966 evaluation

2. Detector accuracy

3. Determination of $n_{s}$

4. $\mathrm{AC}$ effects

$\begin{array}{llll}1.0 & - & 1.0 & - \\ - & 3 & - & 3 \\ - & 2 & - & 2 \\ - & - & 2.0 & - \\ 1.0 & 3.6 & 2.2 & 3.6\end{array}$

C. Calibration-Console

1. Basic accuracy

2. Detector accuracy

3. Determination of $n_{S}$

4. $A C$ effects

$\begin{array}{cccc}1.0 & - & 1.0 & - \\ - & 3 & - & 3 \\ - & 2 & - & 2 \\ - & - & 2.0 & - \\ 1.0 & 3.6 & 2.2 & 3.6\end{array}$

\section{$b_{0}$}

1.0

3.6

2.2

3.6

Limits, $\pm b, p p m$

$1 \mathrm{kHz} \quad 50 \mathrm{kHz} \quad 100 \mathrm{kHz}$

D. MJTC's

1. 1976 assignment of group average

0.3

0.1

2. Possible changes 1976-1982

3. Assignment of $\mathrm{G}-44$

0.1

$b_{0}$

0.33 


$\frac{\text { Limits, } \pm b, \quad p p m}{1 \mathrm{kHz} \quad \underline{50 \mathrm{kHz}} \quad \underline{100 \mathrm{kHz}}}$

E. $5 \mathrm{~mA}$ Reference TE's

1. Residual thermoelectric effects

0.2

0.2

2. High frequency evaluation

$\underline{0.0} \quad \underline{0.5}$

$$
\mathrm{b}_{0}
$$

0.20

0.54

F. $\mathrm{F}_{1} 1 \mathrm{OY}_{2}$, Base TVC

1. $1 \mathrm{kHz}$ base

0.2

0.2

2. High frequency extension

$\underline{0.0}$

1.0

bo

0.2

1.0

G. Reference $T E^{\prime} s$, build-up

to $50 \mathrm{~mA}$ (3 stages)

1. Bead error (each stage)

0.2

0.3

2. Paralleling error (each stage)

$0.1 \quad 0.3$

\section{each stage $b_{0}$}

0.22

0.42

H. Reference $T E^{\prime} s$, build-up from

$50 \mathrm{~mA}$ to $1 \mathrm{~A}$ ( 4 stages)

1. Bead error (each stage)

2. Paralleling error (each stage)

0.2

1.0

3. AC effects (each stage)

0.3

1.0

0.0

1.0

\section{each stage $b_{0}$}

0.36

1.73

I. Reference $T E^{\prime} s$, build-up

$1 \mathrm{~A}$ - 20A (5 steps)

1. Dependence of $d_{c}$ on $I$ (each step)

2. AC effects (induced emf's, etc.) (each step)

3. Proximity effect (each step)

$$
\text { each step } b_{0}
$$

1.0

3.0

0.0

$\underline{2.0}$

2.2

4.1

J. Extension of Voltage Range

10 to $0.5 \mathrm{~V}, 10$ to $200 \mathrm{~V}$

ranges ( 5 steps each)

1. Residual dependence of $d_{c}$

$$
\text { on I (correlated) }
$$

$\underline{0.2}$

$\underline{0.5}$

each step $b_{0}$

0.2

0.5 
Table 15 (Continued)

Limits, $\pm b, p p m$

$1 \mathrm{kHz} \quad 50 \mathrm{kHz} \quad 100 \mathrm{kHz}$

K. Extension of $\mathrm{V}$ range from 200

to $1000 \mathrm{~V}$ range (at $500 \mathrm{~V}$ )

(3 steps)

1. Dependence of $d_{v}$ on $v$

$$
\text { (Avg. for } 3 \text { steps) }
$$

0.5

2.0

2. Dependence of $d_{g}$ on $I$

3. Transfer FX, FY ${ }^{\mathbb{E}}$ to $\mathrm{FX}_{2}$,

0.2

0.5

$\mathrm{FY}_{2}$

0.5

$\underline{2.0}$

each step bo

0.73

2.9

L. Working standard TE's $5 \mathrm{~mA}$ to 20 A range (by comparison with reference stds, same range)

1. Bead resistance and other ac effects

3.0

5.0

$5 \mathrm{~mA}$

6.0

10.0

$20 \mathrm{~A}$

2. Dependence of $d_{c}$ on $I$

$\underline{2.0}$

$\underline{2.0}$

each step bo

$\begin{array}{rrr}3.5 & 5.4 & 5 \mathrm{~mA} \\ 6.3 & 10.1 & 20 \mathrm{~A}\end{array}$

M. Working Standards TVC's ( $F_{7}$ set)

Build-up $10 \mathrm{~V}$ to $1000 \mathrm{~V}$ range

( 4 steps) and build-down 10V

to IV range (3 steps)

1. $d_{c}$ of each TE (correlated)

2. Dependence of $d_{r}$ on $V$ (high ranges), build-up

1.0

3.0

0.0 to

0.0 to

0.5

2.0

3. $\Delta d_{r}$ error (low ranges), build-down

0.0 to

0.0 to

0.5

2.0

average, each step bo

1.1

3.3 


\section{Table 15 (Continued)}

\section{N O T E S :}

\section{Sections}

$A, B, \& C \quad \%$ of $\Delta$ errors depend on magnitude of $\Delta=d_{T}-d_{S}$.

$\mathrm{n}_{\mathrm{s}}$ determined once, but error affects every ac-dc determination. $A C$ effects are estimated residuals or allowances for $\mathrm{rfi}$, induced voltages, etc.

E-1 \& F-1 Main effects evaluated by comparison with MJTC. These are residuals.

G-1 \& H-1 Estimate of error from finite bead resistance.

G-2 \& H-2 Estimate of $\Delta \theta$ error, of paralleled TE's, etc.

I-1 Variation of $d_{c}$ with $I$ was not applied in build-up.

I-3 Proximity of return lead on current distribution in TE heater.

J-1 The error in measuring $\Delta \mathrm{d}_{\mathrm{c}}$ adds at each step.

K-1 Self-heating effect on $d_{r}$.

K-3 Residual substitution error.

M-2 See $\mathrm{K}-1$ note.

M-3 $\Delta \theta$ of $F_{7} T^{\prime}$ 's. See section VIII-B. 
Table 16

$$
\begin{aligned}
& \text { Calculation of Uncertainties } \\
& \mathrm{S}_{\mathrm{e}}=\mathrm{S}_{\mathrm{pa}} \text { or } \mathrm{b} / \sqrt{3}, \mathrm{~S}_{\mathrm{o}}=\left(\Sigma \mathrm{S}_{\mathrm{e}}\right)^{1 / 2}, \mathrm{U}_{\mathrm{o}}=2 \mathrm{~S}_{\mathrm{o}} \\
& \mathrm{S} \text { or } \mathrm{U}(\mathrm{ppm})
\end{aligned}
$$

$1 \mathrm{kHz} \quad 50 \mathrm{kHz} \quad 100 \mathrm{kHz}$

A. MJTC G44

$$
\begin{array}{rrr}
S_{p a} \text { from table 14-1 } & S_{e}=S_{p a} & 0.16 \\
S_{e} \text { for comparator, } & & 0.09 \\
\text { table 15-A } & S_{e}=b / \sqrt{3} & \underline{0.19} \\
S_{e} \text { MJTC'S (15-D) } & S_{e}=b / \sqrt{3} & \\
& U_{A}=2 S_{0}=2\left(\Sigma S_{e}\right)^{1 / 2} & 0.53
\end{array}
$$

B. Reference $5 \mathrm{~mA} T E^{\prime} \mathrm{s} \& \mathrm{~F}_{1} 10 \mathrm{Y}_{2}$

$$
\text { TVC to } 100 \mathrm{kHz}
$$

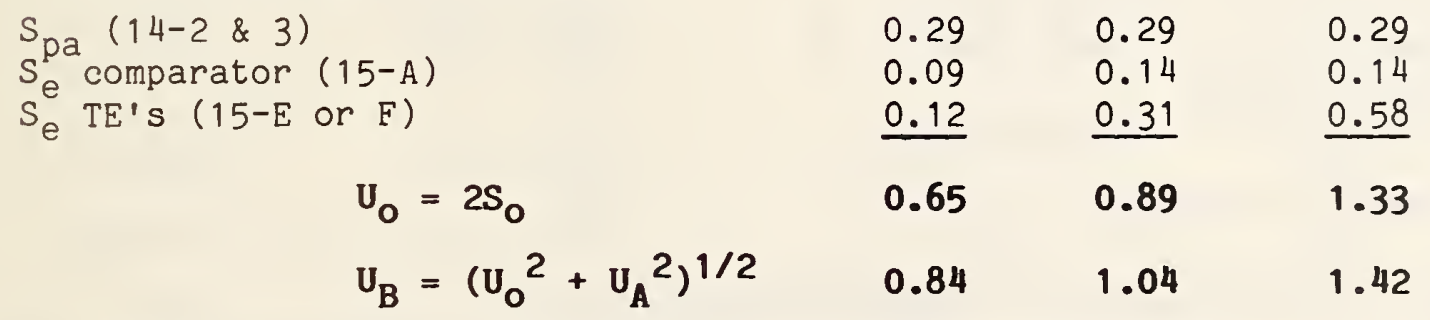

C. Reference TE's 10 to $50 \mathrm{~mA}$ (3 stages)

$\begin{array}{cccc}\mathrm{S}_{\mathrm{pa}}(14.4 \mathrm{a}) & \text { per comparison } & 0.23 & 0.26 \\ \mathrm{~S}_{\mathrm{e}} \text { comp. (15-A) } & \text { per comparison } & 0.09 & 0.14 \\ \mathrm{~S}_{\mathrm{e}} \mathrm{TE} \mathrm{S}(15-\mathrm{G}) & \text { per comparison } & \underline{0.13} & \underline{0.24} \\ \text { Because of triads, } \mathrm{U}_{\mathrm{O}}=0.8 \times 2 \mathrm{~S}_{\mathrm{O}} & 0.45 & 0.61 & \text { per stage } \\ \text { After 3 stages } \mathrm{U}_{03}=\sqrt{3} \mathrm{U}_{\mathrm{O}} & 0.78 & 1.05 \\ \text { After 3 stages } \mathrm{U}_{\mathrm{C}}=\left(\mathrm{U}_{03}{ }^{2}+\mathrm{U}_{\mathrm{B}}{ }^{2}\right)^{1 / 2} & \mathbf{1 . 1} & \mathbf{1 . 5}\end{array}$

D. Reference TE's $100 \mathrm{~mA}$ to $1 \mathrm{~A}$ ( 4 stages)

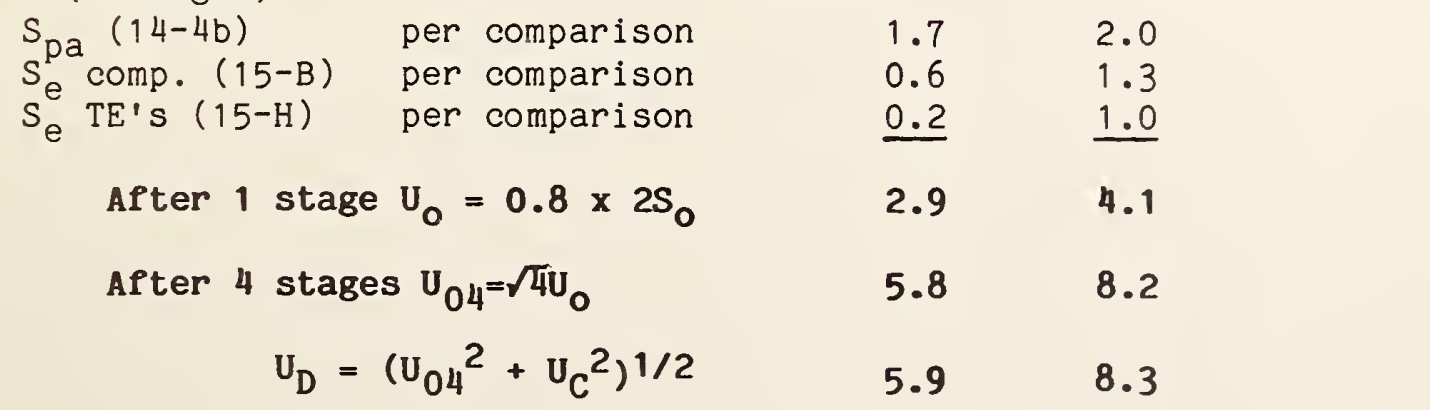


Table 16 (Continued)

$\frac{\mathrm{S} \text { or } \mathrm{U}(\mathrm{ppm})}{1 \mathrm{kHz} \quad 50 \mathrm{kHz} \quad 100 \mathrm{kHz}}$

E. Reference TE's $2 \mathrm{~A}$ to 20A (at 10A)

$$
\text { (5 steps) }
$$

$\begin{array}{ll}S_{p a}(14-4 C) & \text { per step } \\ S_{e} \text { comp. }(15-B) & \text { per step } \\ S_{e} T E^{\prime} S(15-I) & \text { per step }\end{array}$

\begin{tabular}{ll}
1.6 & 1.7 \\
0.6 & 1.3 \\
1.3 & $\underline{2.4}$ \\
\hline 4.3 & 6.4 \\
9.6 & 14 \\
11 & 16
\end{tabular}

F. F $F_{1}$ TVC's 10 to $0.5 \mathrm{~V}$ and 10 to $100 \mathrm{~V}$ ( 6 and 4 steps)
After 1 step $\mathrm{U}_{\mathrm{O}}=2 \mathrm{~S}_{\mathrm{O}}$
After 5 steps $U_{05}=\sqrt{5} U_{0}$
$U_{E}=\left(U_{05}{ }^{2}+U_{D}{ }^{2}\right)^{1 / 2}$

$S_{p a}(14-5 a)$ each step

$S_{e}^{p a}$ comp. (15-A) each step

$\begin{array}{ll}\text { a } & 0.47\end{array}$

0.54

0.09

0.14

$S_{e}$ TVC's $(15-J)$

b

$\underline{0.11}$

$\underline{0.29}$

After 1 step $U_{0}=2 S_{0}$

0.98

1.26

After 5 steps $U_{05^{2}}=2\left((5 c)^{2}+5(a+b)^{2}\right)^{1 / 2}$

2.7

4.2

$U_{F}=\left(U_{05}{ }^{2}+U_{B}^{2}\right)^{1 / 2}$

2.8

4.4

G. F 1 TVC's 200 to $1000 \mathrm{~V}$ range (at 500V) (3 steps)

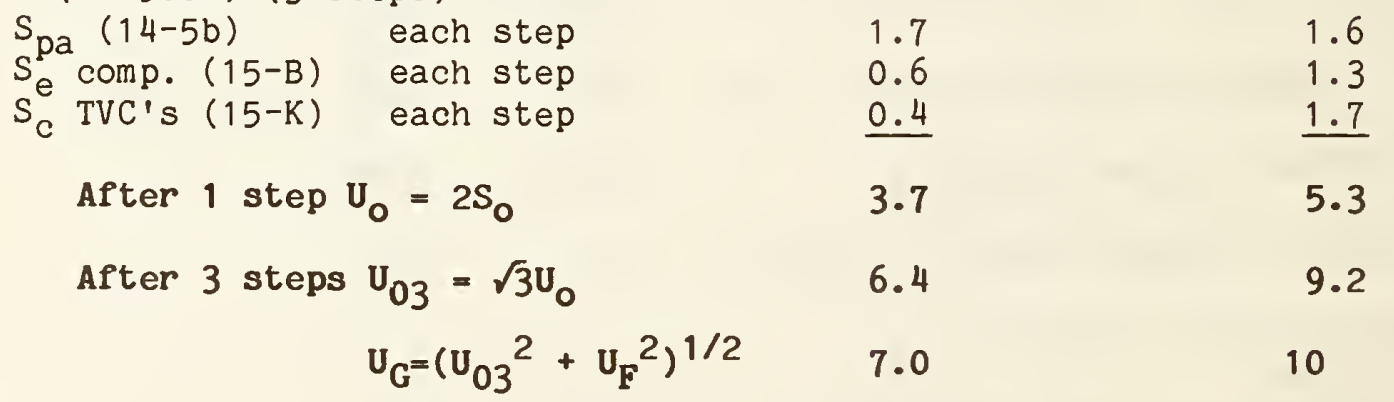


Table 16 (Continued)

$\frac{\mathrm{S} \text { or } \mathrm{U}(\mathrm{ppm})}{1 \mathrm{kHz} \quad 50 \mathrm{kHz} \quad 100 \mathrm{kHz}}$

H. Working TE's $5 \mathrm{~mA}$ to $20 \mathrm{~A}$ range $S_{\text {pa }}(14-6)$

$S_{e}^{p a}$ comp. $(15-c)$

$S_{e} T E^{\prime} S(15-L) \quad 5 \mathrm{~mA}$

$S_{e}$ Reference TE's (16-B) 5mA

$(16-E) 20 A$

$2.8 \quad 1.7$

$0.6 \quad 1.2$

$2.1 \quad 3.1$

$3.6 \quad 5.8$

$0.4 \quad 0.5$

$\underline{5.6} \quad \underline{8.2}$

$\begin{array}{cccc}\mathrm{U}_{\mathrm{H}}=2 \mathrm{~S}_{\mathrm{o}} & 5 \mathrm{~mA} & 7.1 & 7.5 \\ 20 \mathrm{~A} & 15 & 21\end{array}$

I. $F_{7}$ Working Standard TVC's

10 to $1000 \mathrm{~V}$ range (5 steps);

10 to $1 \mathrm{~V}$ range (3 steps)

$S_{\text {pa }}(14-7) \quad$ each step

$\mathrm{S}_{\mathrm{e}}^{\mathrm{pa}}$ comp. (15-C) each step

a 1.2

2.8

0.6

1.2

$S_{e}$ TVC's (15-M)

each step

$\underline{0.6}$

1.9

After 1 step $U_{0}=2 S_{0}$

2.9

7.2

After 3 steps $\mathrm{U}_{03}=2\left((3 c)^{2}+3(a+b)^{2}\right)^{1 / 2}$

7.2

18

$$
\text { (at 1V) } \quad U_{I}=\left(U_{03}^{2}+U_{B}{ }^{2}\right)^{1 / 2}
$$

7.2

18

After 5 steps $U_{05}=2\left((5 c)^{2}+5(a+b)^{2}\right)^{1 / 2}$

10

26

$$
\text { (at 500V) } \quad U_{I}=\left(U_{05}{ }^{2}+U_{B}{ }^{2}\right)^{1 / 2}
$$

10

26 


\section{Summary of Uncertainties of NBS AC-DC Transfer Standards}

I. Uncertainties Independent of Magnitude of $d$

$\mathbf{U}_{\mathrm{A}}$ ppm

Standard

$1 \mathrm{kHz}$

$50 \mathrm{kHz}$

$100 \mathrm{kHz}$

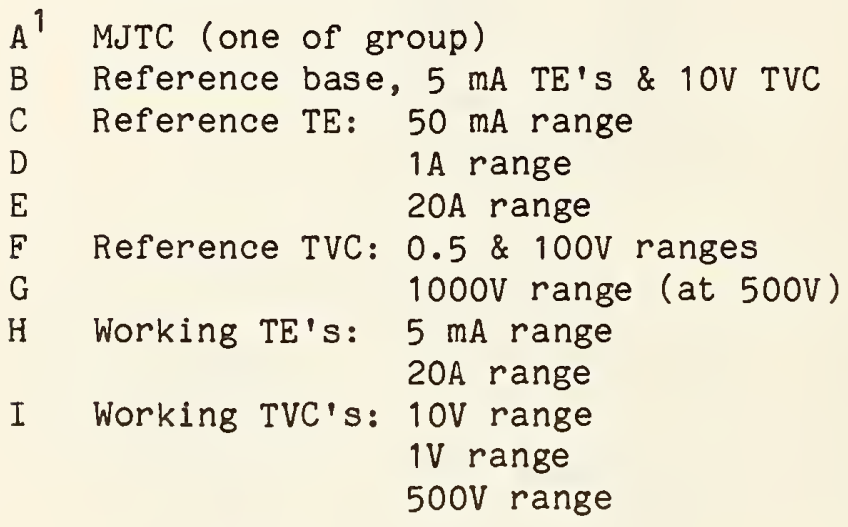

0.53

0.84

1.1

1.0

1.4

5.9

11

1.5

8.3

2.8

7.0

7.1

15

16

II. Uncertainties dependent on magnitude of $d$ :

For all standards $U_{B}=0.05 d$ should be added to $U_{A}$. $U_{B}<1$ ppm with few exceptions.

III. Uncertainties at $20 \mathrm{~Hz}$ are somewhat, but not significantly, greater than at $1 \mathrm{kHz}$.

IV. Uncertainties at less than rated $V$ or $I$ require further study. However, the working standards are almost always used at or near rated $V$ or $I$.

V. Self-heating effects at $1000 \mathrm{~V}$ were evaluated in [3].

Letters refer to table 16. 


\section{Table 18}

\section{Comparison of Estimated Calibration Uncertainties with}

\section{Present Allowances}

\section{$U(\mathrm{ppm})$}

$\frac{1 \mathrm{kHz}}{5 \mathrm{~mA} \quad 20 \mathrm{~A}} \frac{50 \mathrm{kHz}}{5 \mathrm{~mA} \quad 20 \mathrm{~A}}$

Working standard TE's (16-H)

Comparison (16-H without $\mathrm{S}_{\mathrm{e}}$ of ref. stds.)

$\begin{array}{llll}7.1 & 15.0 & 7.5 & 21 \\ 7.1 & \underline{9.2} & \underline{7.5} & \underline{12} \\ 10 & 18 & 11 & 24\end{array}$

\section{U (rss)}

50

100

100

Present allowance $5 \mathrm{~mA}-5 \mathrm{~A}$

Present allowance 5A-20A

$\frac{1 \mathrm{kHz}}{\frac{1 \mathrm{~V} \quad \text { 10V } \quad \text { 500V }}{1 \mathrm{~V} \quad \text { 10V } \quad 500 \mathrm{~V}}}$

Working standard TVC's (16-I)

Comparison (16-I, one step)

\section{U (rss)}

Present allowance (for coaxial TVC's )

Present allowance (for multirange TVC's) $\begin{array}{lll}7.2 & 3.0 & 10 \\ 2.9 & 2.9 & 2.9\end{array}$

$\begin{array}{lll}7.8 & 4.2 \quad 10\end{array}$

$20 \quad 20$

20

50

50

50 $\begin{array}{lll}18 & 7.2 \quad 26\end{array}$

$\begin{array}{lll}7.2 & 7.2 & 7.2\end{array}$

$19 \quad 10 \quad 27$

$50 \quad 50 \quad 50$

$100 \quad 100 \quad 100$

NOTE: This table applies to the evaluation in the NBS Calibration Console of single-junction TVC's and single-range TE's of the same quality as the NBS standards. 
Table 19

Estimated Uncertainties for Special Tests

(Calibration of a TE or TVC against a reference standard, with MJTC comparator)

\begin{tabular}{|c|c|c|c|c|c|c|}
\hline & & & $\mathbf{U}$ & & & \\
\hline & & & $\mathrm{zz}$ & & $\mathrm{kHz}$ & \\
\hline & & $5 \mathrm{~mA}$ & $50 \mathrm{~mA}$ & & & $\mathrm{~mA}$ \\
\hline Reference standard TE $5-50 \mathrm{~mA}$ & & 0.9 & 1.1 & 1 & & \\
\hline $\begin{array}{l}\text { Comparison ( } 16 \mathrm{~B} \& 16 \mathrm{C} \text { without } \mathrm{S}_{e} \\
\text { of TE's) }\end{array}$ & & $\underline{0.6}$ & $\underline{0.6}$ & $\underline{0}$ & & \\
\hline rss & & 1.1 & 1.2 & 1 & & \\
\hline & & $1 \mathrm{kHz}$ & & & $100 \mathrm{kH}$ & \\
\hline & $\underline{1 \mathrm{~V}}$ & $10 \mathrm{~V}$ & $100 \mathrm{~V}$ & 1V & $10 \mathrm{~V}$ & $100 \mathrm{~V}$ \\
\hline $\mathrm{F}_{1} \mathrm{TVC} \quad 0.5-100 \mathrm{~V}$ & 2.8 & 0.9 & 2.8 & 4.4 & 1.4 & 4.4 \\
\hline Comparison (16-C, all ranges) & 0.6 & 0.6 & 0.6 & 0.6 & 0.6 & 0.6 \\
\hline Substitution uncertainty & 0.5 & $\underline{0.5}$ & 0.5 & 2.0 & 1.0 & 2.0 \\
\hline rss & 2.9 & 1.2 & 2.9 & 4.9 & 1.8 & 4.9 \\
\hline
\end{tabular}




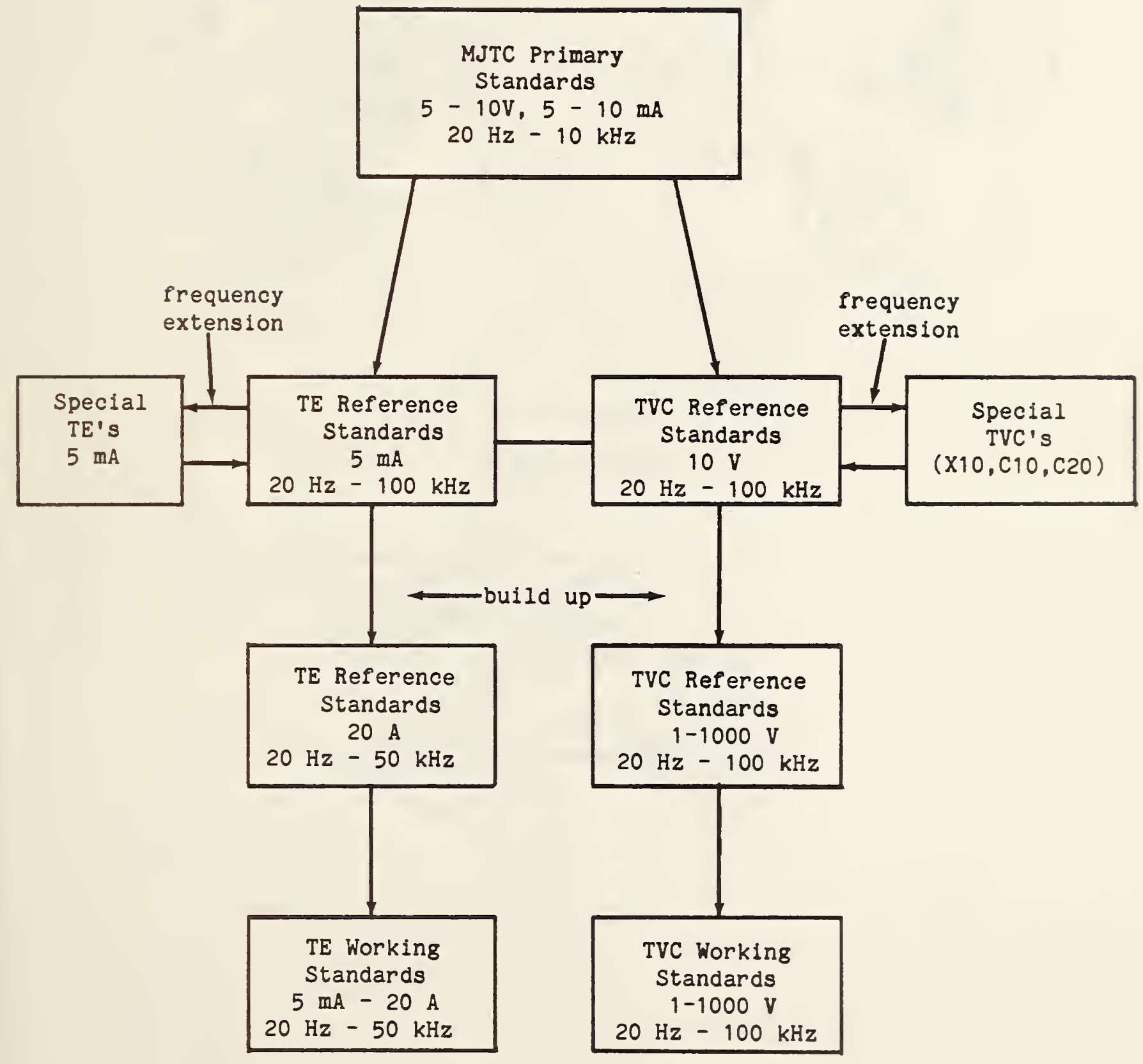

Figure 1. "Road Map" 
$30 \mathrm{~Hz}$

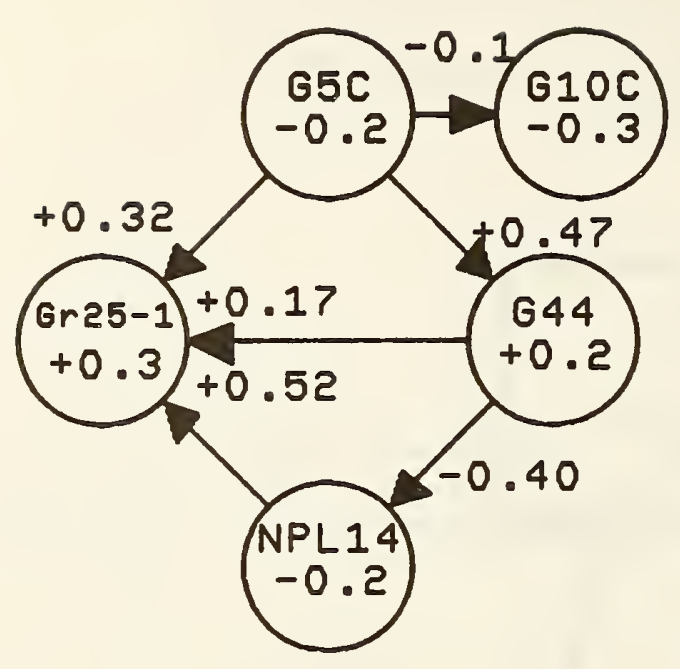

$1 \mathrm{kHz}$

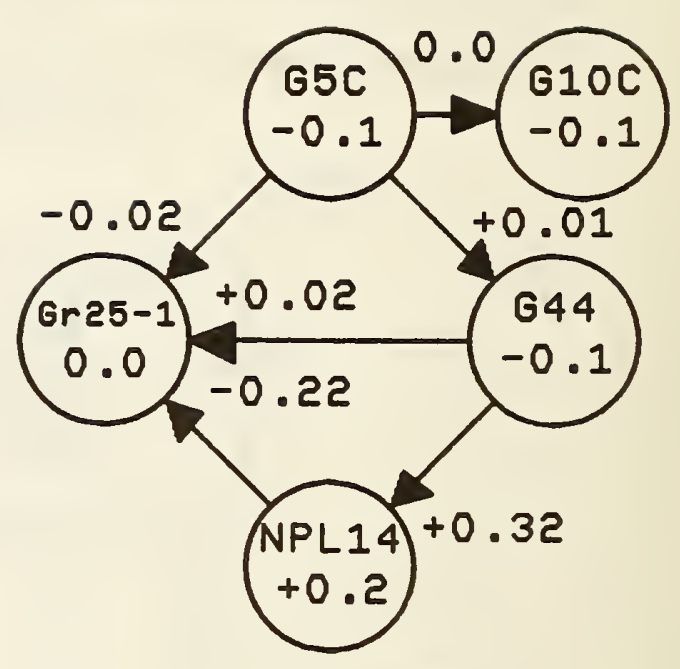

$10 \mathrm{kHz}$

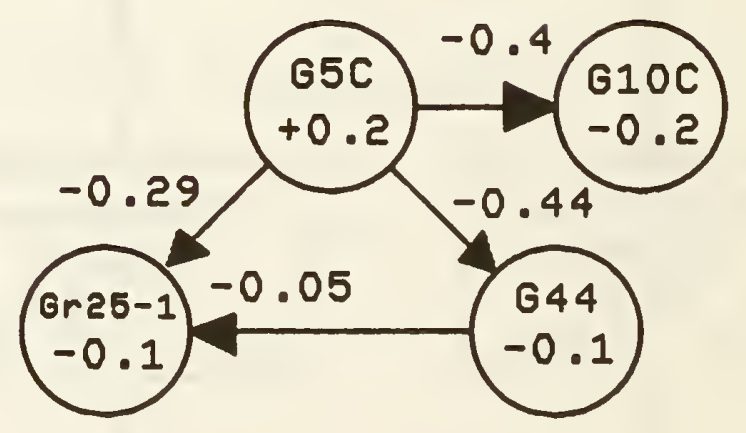

Figure 2. Comparisons of MJT's 


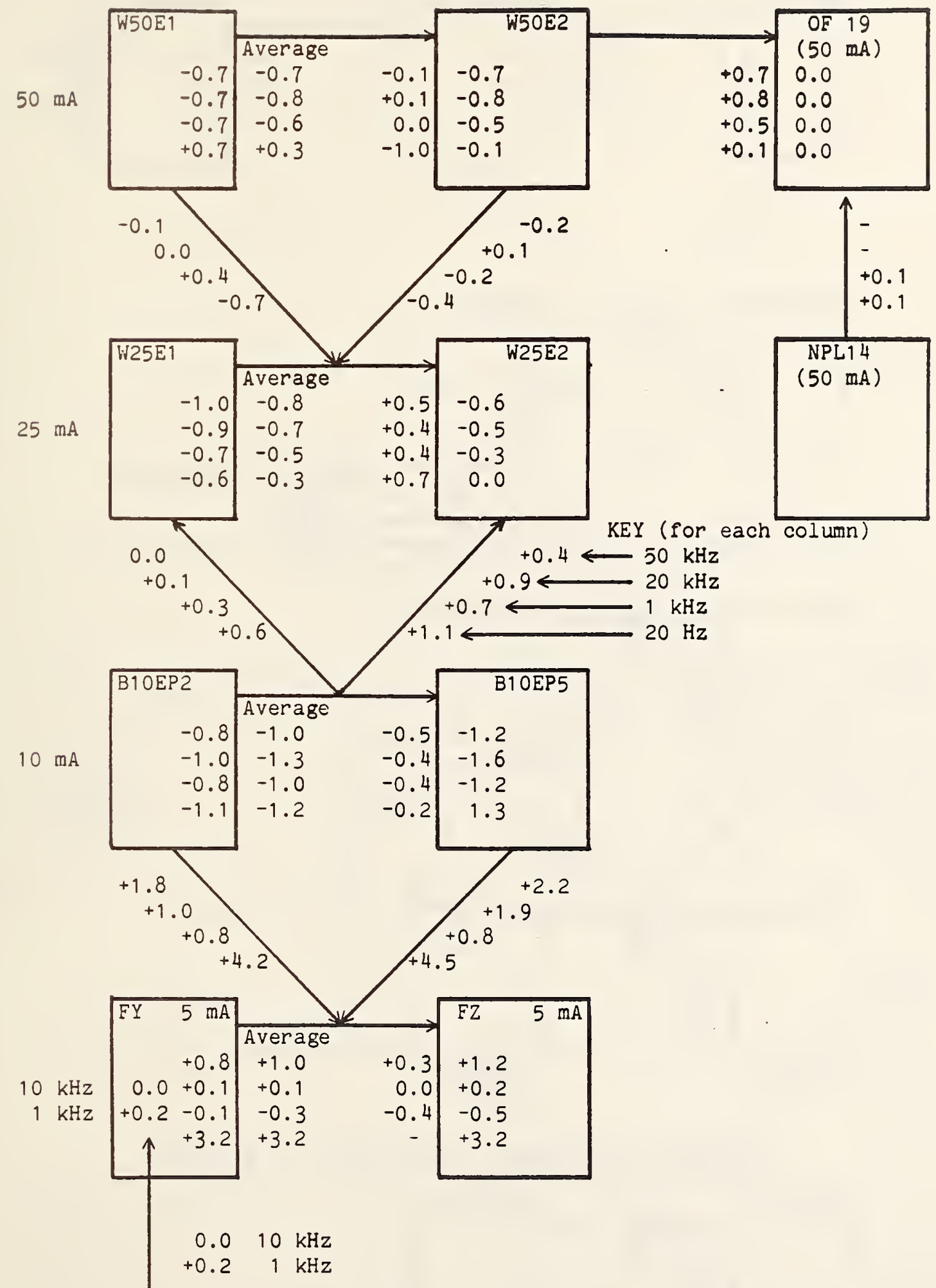

Figure 3: $50 \mathrm{~mA}$ to $5 \mathrm{~mA}$ Comparisons 


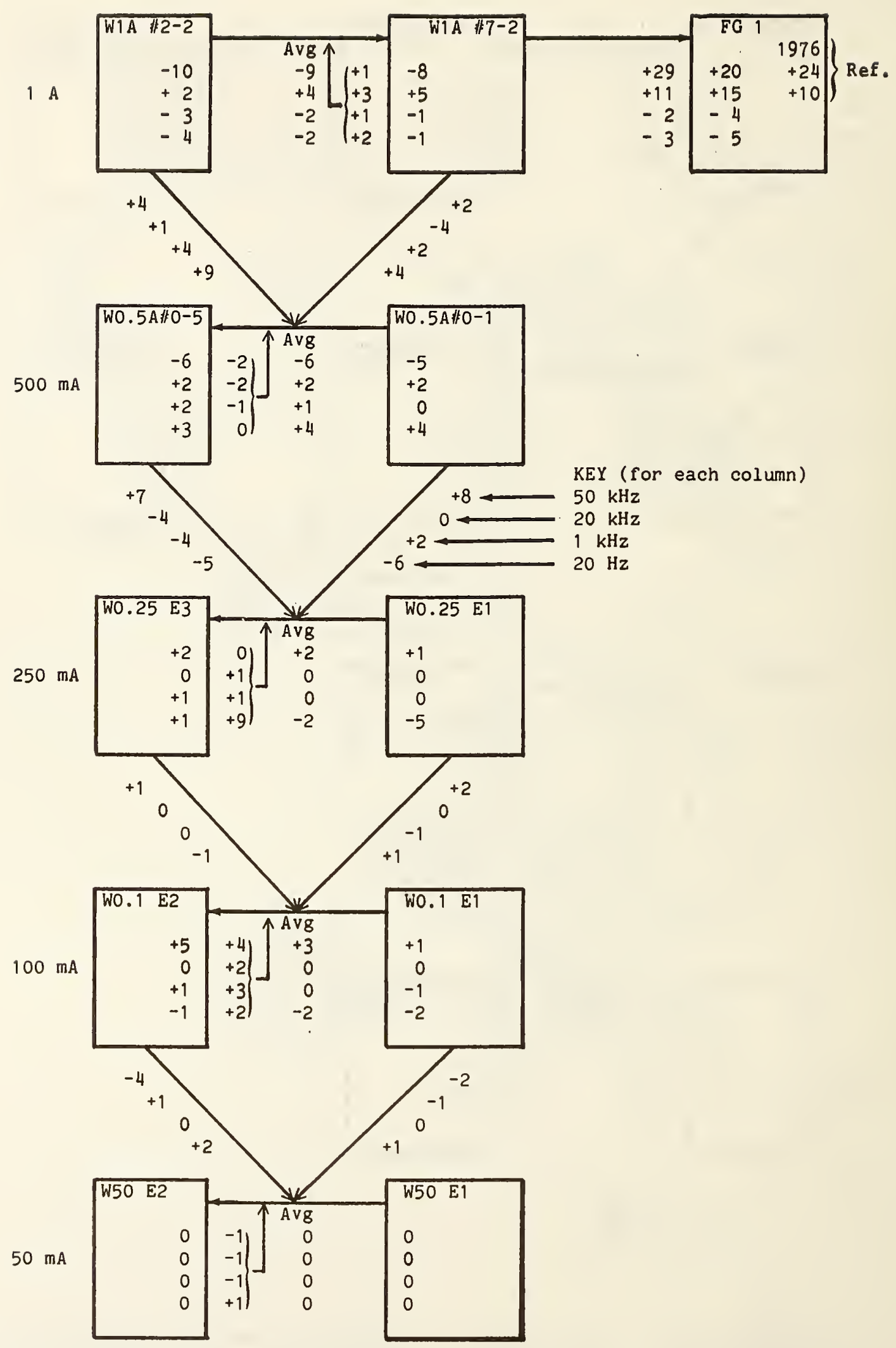

Figure. 4: $50 \mathrm{~mA}$ to 1 A Comparisons 


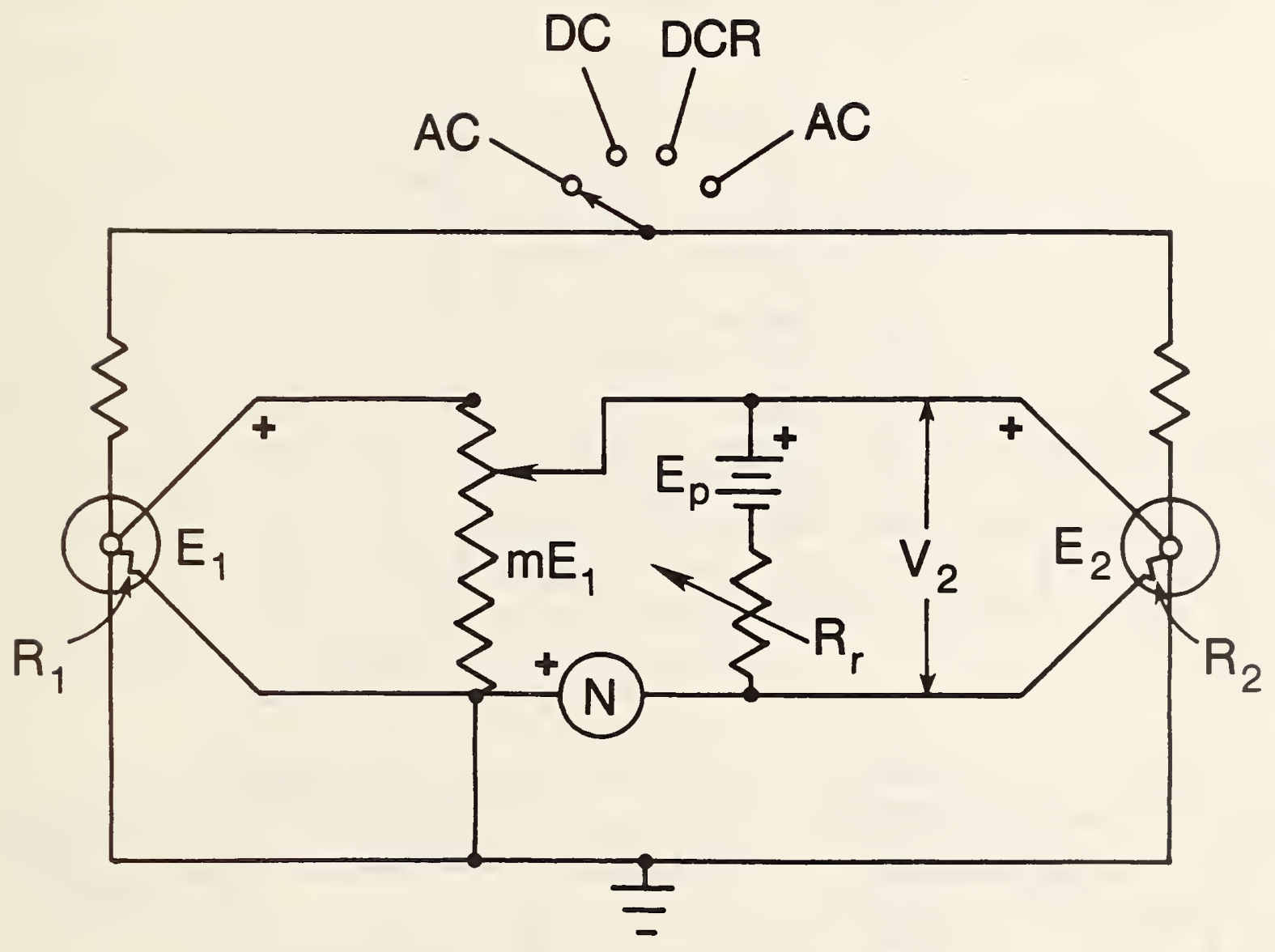

Figure 5. Comparator With n-Compensation 


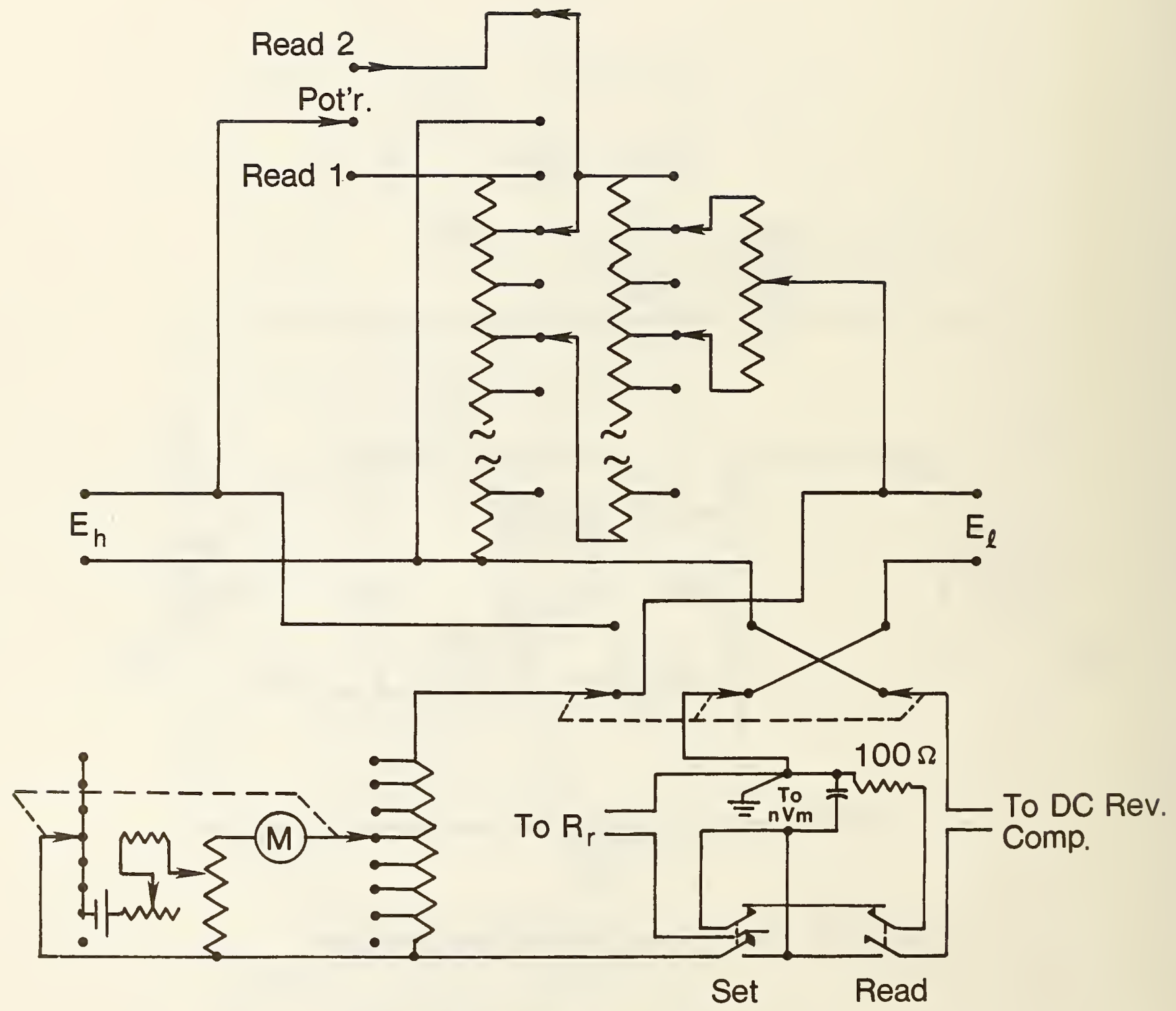

Figure 6. Diagram of EMF Comparator 


\section{APPENDIX 1}

\section{n-COMPENSATION and n-MEASUREMENTS}

\section{A. Principle of Compensation}

The basic circuit of the M.JTC comparator with the $n$-compensation resistor, $R_{r}$, included is shown in figure $5 . E_{p}$ represents an adjustable Lindeck potentiometer.

At the initial balances, with $\mathrm{N}=0$, ( $N$ is the reading of the high impedance nanovoltmeter, $n V m$ ), $m E_{1}=E_{2}=E_{p}=V_{2}$. (The $E_{p}$ setting is made by switching $n V m$ in place of $R_{r}$ ) Now if the input voltage $V_{i}$, is increased by a small amount, $\Delta V_{i}$, then $\Delta E_{1} / E_{1}=n_{1} \Delta V_{i} / V_{i}$ and $\Delta E_{2} / E_{2}=n_{2} \Delta V_{i} / V_{i}$ (from the definition of $n$ given in section IIIA with $V_{i}$ replacing $I$ ). A small current then flows in the $E_{2}-E_{p}$ loop. Because of the voltage divider formed by $R_{2}$ and $R_{r}, \Delta V_{2}$ is less than $\Delta E_{2}$. Thus if $n_{2}$ is greater than $n_{1}, R_{r}$ can be adjusted until $m \Delta E_{1}=\Delta V_{2}$, maintaining $N=0$. This makes the steady-state balance insensitive to small changes in the supply voltages, and makes it unnecessary to adjust these voltages to keep one of the emf's constant during an ac-dc determination, as heretofore required.

From the figure and with the output resistance of the potentiometer included in $R_{r}, \Delta V_{2}=q \Delta E_{2}$, where $q=R_{r} /\left(R_{r}+R_{2}\right)$. Since the same $\Delta V / V$ is applied to both TVC's

$$
\frac{\Delta E_{1}}{n_{1} E_{1}}=\frac{\Delta E_{2}}{n_{2} E_{2}}=\frac{m \Delta E_{1}}{q n_{2} m E_{1}} \text {, so that } q=\frac{n_{1}}{n_{2}}
$$




\section{B. Basic TE Equation}

Since neither TE has a constant output during a comparison of two TVC's (or TE's) the development of the comparator equation given in appendix 2 of [3] is not directly applicable. It will be convenient to use a general equation for a TVC with slightly different ac and dc input voltages, $V_{a}$ and $\mathrm{V}_{\mathrm{d}}$, applied. We may write, as an identity,

$$
v_{a}=v_{d}\left(1+\frac{v_{d}^{\prime}-v_{d}}{V_{d}}+\frac{v_{a}-v_{d}^{\prime}}{v_{d}}\right)
$$

where $V_{d}$ is the average of the two directions of dc voltage required to produce the same emf as $V_{a}$, and $V_{d}$ is the average of the two directions which produce an emf, $E_{\mathrm{d}}$.

Then, from the definitions of $n$ and $d$, and, since $E_{a}=E_{d}{ }^{\prime}$,

$$
v_{a}=V_{d}\left(1+\frac{E_{a}-E_{d}}{n E_{d}}+d\right) \text {, to 1st-order terms. }
$$

A similar equation applies for current measurements, with I replacing $V$.

\section{AC-DC Comparisons}

From the above basic TVC equation, and with the same voltage applied to both TVC's, as in fig. 5 .

$$
v_{a}=v_{d}\left(1+\frac{E_{1 a}-E_{1 d}}{n_{1} E_{1 d}}+d_{1}\right)=v_{d}\left(1+\frac{E_{2 a}-E_{2 d}}{n_{2} E_{2 d}}+d_{2}\right)
$$

Thus

$$
d_{1}-d_{2}=\frac{E_{2 a}-E_{2 d}}{n_{2} E_{2}}-\frac{E_{1 a}-E_{1 d}}{n_{1} E_{1}}
$$


But $N_{a}=V_{2 a}-m E_{1 a}$ and $N_{d}=V_{2 d}-m E_{1 d}$, with the polarity shown, so that $N_{a}-N_{d}=V_{2 a}-V_{2 d}-m\left(E_{1 a}-E_{1 d}\right)=q\left(E_{2 a}-E_{2 d}\right)-m\left(E_{1 a}-E_{1 d}\right)$. Combining these equations, and noting that $q=n_{1} / n_{2}$ and $m E_{1}=E_{2}$, gives, closely enough,

$$
d_{1}-d_{2}=\frac{\left(E_{2 a}-E_{2 d}\right)}{n_{1} E_{2}}-\frac{m\left(E_{1 a}-E_{1 d}\right)}{n_{1} E_{2}}=\frac{N_{a}-N_{d}}{n_{1} E_{2}}
$$

If $n_{1}>n_{2}$, the potentiometer and $R_{r}$ are connected across $E_{1}$, and the polarity of the nanovoltmeter is reversed. $R_{r}$ is adjusted as before, so that there is no change in $N$ when $V_{i}$ is increased. Because of the input resistance, $R_{d}$, of the divider, analysis shows that

$$
\frac{n_{2}}{n_{1}}=\frac{R_{p}}{R_{p}+R_{1}} \quad \text { where } R_{p}=\frac{R_{r} R_{d}}{R_{r}+R_{d}}
$$

The comparator formula then becomes

$$
d_{2}-d_{1}=\frac{N_{a}-N_{d}}{n_{2} E_{2}}
$$

Equations $A 1 B-1$ and $A 1 B-2$ may be written as

$$
d_{T}-d_{S}=\frac{N_{d}-N_{a}}{n_{S} E_{l}}
$$

where the subscript $T$ refers to the $T E$ to which the potentiometer is connected, and $E_{\ell}$ refers to the TE with the lowest emf. 


\section{Precautions in $n$-Measurement}

The characteristic, $n$, is conveniently measured, after the initial balance is obtained with the set key down, by changing the input quantity, $Q$, by about $0.1 \%$, noting the change in output emf $E$, then calculating $n=Q \Delta E / E \Delta Q$. The usual precautions in measuring small changes should be observed, as in an ac-dc difference test, to avoid errors from drifts etc.

A more insiduous error can occur, however, if the heater alloy of a TE has even a small temperature coefficient of resistance, $\alpha$, so that $\mathrm{R}=\mathrm{R}_{\mathrm{O}}(1+\alpha \theta)$, where $\theta$ is the temperature $\mathrm{rise}$ above ambient. Then, since $\mathrm{V}=\mathrm{IR}$ for the heater, and $\theta=k I^{2}$

$$
\begin{aligned}
\frac{d V}{V} & =\frac{d I}{I}+\frac{d R}{R}=\frac{d I}{I}+\frac{d R}{d I} \frac{d I}{R}=\frac{d I}{I}+\frac{2 R_{O} \alpha k I d I}{R} \\
& =\frac{d I}{I}(1+2 \alpha \theta) \text { since } R_{0}=R
\end{aligned}
$$

From the definition of $\mathrm{n}$ and the above equation

$$
n_{i}=n_{v}(1+2 \alpha \theta) \text {. }
$$

For the NiCr alloys normally used in low-range TEs, $\alpha$ can be $-1 \times 10^{-4}$ to $-2 \times 10^{-4}$. $\theta$ is usually about $1.5 \times 10^{2}$ at rated $I$. Thus $n_{v}$ and $n_{1}$ can differ by 3 to $6 \%$. For alloys like Evanohm the difference is much smaller.

Thus the appropriate $\mathrm{n}$ should be measured and used. Since it enters only in a difference term with this comparator, small errors in determining it are not significant unless $d_{T}{ }^{-d_{S}}$ is large. 


\section{E. Square Law Output and RMS Response}

It is worth emphasizing that even if $n \neq 2$, so that $E \neq K I^{2}$ with $K a$ constant, a TE inherently responds to the true rms current, I. This can be shown easily for the simplest case in which all of the heater is at the same constant temperature, $\theta$. Then, equating heat input and output at any instant, $i^{2} R=K_{1} \theta=K_{2} E . R, K_{1}$ and $K_{2}$ are generally somewhat dependent on $\theta$, but if the frequency of the heater current, $i$, is high enough, thermal inertia keeps $\theta$, and hence $E$ constant. Then term by term integration with respect to time, $t$, over one period, $T$, and division by $T$, gives, directly, $I^{2} R=K_{2} E$. Because of the temperature dependence of $R$ and $K_{2}$ the output is not exactly proportional to $I^{2}$, but it is still a measure of $I$, the rms current. This can also be shown to be true under far more general conditions (see appendix 3 of [1]). 



\section{Appendix 2}

\section{EMI, Harmonics and DC Offset}

\section{A. Test for Line-Borne Electromagnetic Interference (EMI)}

Only about $1 \mu \mathrm{W}$ of heater power, over a wide range of frequency, can produce $0.1 \%$ of rated emf in a typical 5-mA TE. Thus TE's are sensitive to EMI, particularly at TV and FM frequencies. (They were the detectors in early field-strength meters.) Laboratory leads at these frequencies can transfer radiated energy fairly efficiently to the heaters. Of course if the leads are twisted or shielded they make very poor antennas. However shielding is not completely effective, and in areas of high field strength (up to $1 \mathrm{~V} / \mathrm{m}$ in some areas) errors can result if the TE responds to currents at both the main and interfering frequency.

However, because of their square-law characteristics ( $E=\mathrm{KI}^{2}$ where $\mathrm{I}$ is the rms current) a small heater current, $I_{e}$, at a different frequency than the main current, $I_{m}$, does not cause as large an error as the ratio $a=I_{e} / I_{m}$ might indicate.

$$
I=\left(I_{m}^{2}+I_{e}^{2}\right)^{1 / 2}=I_{m}\left(1+a^{2}\right)^{1 / 2}
$$

If the response sensitivity is the same at both frequencies

$$
E=K I^{2}=K I_{m}^{2}\left(1+a^{2}\right)
$$

Without $I_{e}, E_{m}=K I_{m}^{2}$. Thus $\left(E-E_{m}\right) / E_{m}=\Delta E / E_{m}=a^{2}$. With only $I_{e}$ present, $E_{e}=K I_{e}^{2}$ so that $E_{e} / E_{m}=a^{2}$.

If a measurement of $I_{m}$ is desired, $\Delta E / E_{m}$ is an error term. It can be evaluated to see if it is significant by making a measurement with all of the connections in place but with the power supplies producing $I_{m}$ turned off. 
In the more general case $K$ is somewhat dependent in $E$, so that the sensitivity of this test is affected slightly. Also, if both TE's in an ac-de comparison respond equally well to currents at both frequencies, there will be no EMI error. However the EMI test will determine this also.

In the MJTC comparator, RF bypass capacitors (of very low inductance) are connected from each ungrounded lead to the metal tank to reduce the possibility of EMI errors. The tank itself is an excellent shield.

\section{B. Effect of Harmonics (see Section VII)}

Small amounts of low-order harmonics in the input voltage generally cause insignificant errors in the measurement of the rms value by a TVC, or in the comparison of two TVC's. This is a great advantage for true rms-measuring instruments. This can be shown rather simply for a wave having a fundamental voltage, $v_{1}$, and a single harmonic, $v_{h}$. Then $V=\left(v_{1}{ }^{2}+v_{h}{ }^{2}\right)^{1 / 2}$. Because of reactance, skin effect, etc., the response of the TVC may be different at the two frequencies, so that $E=k V^{2}=k_{1} V_{1}^{2}+k_{h} V_{h}^{2}$. Let $V_{h} / V_{1}=q$ and $K_{h}=K_{1}\left(1+e_{h}\right)$. Then $E=k_{1} V^{2}\left(1+q^{2} e_{h}\right)$ so that the proportional error is only $q^{2} e_{h}$.

For several harmonics this becomes $\sum q_{m}{ }^{2} e_{m}$, where $m$ refers to the order of the harmonic.

\section{DC offset}

The above equations should apply even if the harmonic is of zero order (dc). However if the TE has a dc reversal difference (which can be caused by Thomson or Peltier heating) there is an added output, $E=K_{0} V_{O S}$, proportional to the first power of any de offset voltage, $V_{\text {os }}$, in the ac wave, $V_{a}$. Then

$$
E=k\left(v_{a}^{2}+v_{o s}^{2}\right)^{1 / 2}+K_{o} v_{o s}=K V^{2}\left(1+\frac{K_{o} V_{o s}}{E}\right)
$$


With a dc voltage, $v_{d}$, equal to $V$ applied and then reversed, the dc reversal difference in output is $\Delta E=2 K_{O} V$. Thus

$$
E=k v^{2}\left(1+\frac{g u}{2}\right)
$$

where

$$
g=\frac{\Delta E}{E} \text { and } u=\frac{V_{\text {OS }}}{V}
$$

Note that this is proportional to the 1 st power of $u$, and that, for a square law TE, $g$ is twice the value of the proportional de reversal difference as normally defined $(\Delta V / V$ for constant $E)$. 



\section{Appendix 3}

\section{Effect of Relay off-Time}

Let the response, $E$, of a TE be approximated by a simple exponential with a time constant, $t_{c}$. Then after the steady-state heater current is turned off for a time $t_{0}<t_{c}$ (the off-time of the relay when switched), we have

$$
E=E_{f} e^{-t_{0} / t_{c}} \approx E_{f}\left(1-t_{o} / t_{c}\right)
$$

Thus $\Delta E=E_{f}-E=E_{f} t_{o} / t_{c}$.

When the relay is closed (with the same heater current), E recovers to $E_{f}$ exponentially, so that

$$
E=E_{f}-\Delta E e^{-t_{S} / t_{c}}
$$

where $t_{S}=t-t_{O}$. Then $p=\left(E_{f}-E\right) / E_{f}=b / e^{a}$ where $b=\Delta E / E_{f}=t_{o} / t_{c}$ and $a=t_{s} / t_{c}=t / t_{c}$ when $t_{s} \gg t_{c}$. If $p=1 \times 10^{-6}$ (recovery to $1 \mathrm{ppm}$ ), $t_{0}=$ $4 \times 10^{-4} \mathrm{~s}$, and $t_{c}=2 \mathrm{~s}$, then $b=2 \times 10^{-4}$ and $a=5.3$. Thus $t=a t_{c}=10.4 \mathrm{~s}$.

Interestingly, when $t_{o}=4 \times 10^{-3} \mathrm{~s}, t=15.2 \mathrm{~s}$ to recover to $1 \mathrm{ppm}$, only a modest increase for this 10-fold increase in of $t$ ime. 


\section{Appendix 4}

\section{Effects of Bead Heating}

\section{A. Relative Effectiveness of Bead and Heater Power}

Widdis has shown (equation 17 of [19]) that the midpoint temperature rise, $\theta_{m}$, of the heater of a TE carrying a current, $I$, taking into account the heat loss through the thermocouple wires as well as the heater, to heat sinks at the ends $($ all at $\theta=0)$ is $\theta_{m}=D \ell^{2} / 2 M$. Here the very small Peltier and Thomson heating is neglected, $D=I^{2} \rho / A^{2} K, \rho$ and $K$ are the electrical resistivity and thermal conductivity of the heater, $A$ is the cross sectional area and $2 l$ the length of the heater, and $M=1+K_{1} / K_{0}$, where $K_{1}$ is the sum of the thermal conductances of the two thermocouple wires in parallel and $K_{0}$ is the sum of the conductances of the two halves of the heater.

$$
\begin{aligned}
& \text { Since } k_{0}=2 \mathrm{KA} / \ell \text { and } 2 \rho \ell / A=R_{h} \text {, the heater resistance, we find that } \\
& \theta_{m}=\frac{P_{h}}{2\left(K_{0}+K_{1}\right)}
\end{aligned}
$$

where

$$
P_{h}=I^{2} R_{h}
$$

If the bead between the heater and the hot junction of the thermocuple has a resistance $R_{b}$, its power dissipation is $P_{b}=I_{b}{ }^{2} R_{b}$. The added temperature $r$ ise from this small heating is $\Delta \theta=P_{b} /\left(K_{1}+K_{0}\right)$. Thus bead power is twice as effective as heater power in raising the temperature of the hot junction. 


\section{B. AC-DC Difference Caused by Bead Heating}

One end of the heater of a TVC is grounded, and the thermocouple circuit is usually grounded also for safety and to keep the bead voltage low. Thus half the heater voltage is across the bead. From the formula in section $V-B$ the significant current is $I_{1}$ through the ungrounded heater terminal. The heater power is, to first order terms,

$$
P_{h}=\frac{I_{1}{ }^{2} R_{h}}{2}+\frac{I_{1}{ }^{2} R_{h}}{2}(1-a)^{2} \approx I_{1}{ }^{2} R_{h}(1-a)
$$

where

$$
a=\frac{I_{b}}{I_{1}}=\frac{R_{h}}{2 R_{b}} \ll 1
$$

The temperature rise, with the effect of bead power included, is

$$
\theta=U P_{h}+2 U P_{b}=U I_{1}{ }^{2} R_{h}(1-a)+2 U a^{2} I_{1}{ }^{2} R_{b}
$$

where

$$
U=1 / 2\left(k_{0}+k_{1}\right)
$$

Thus

$$
\theta=U I_{1}{ }^{2} R_{h}\left(1-a+2 a^{2} R_{b} / R_{h}\right)=U I_{1}{ }^{2} R_{h}
$$

with no error from the bead current, to first order terms.

This will, of course, not be true if neither heater terminal is at ground. 
C. Two TE's in Series

A similar but more involved analysis shows that if two equal series-connected TE's are used in a grounded TVC, the resultant output will also be unaffected by the bead currents. 


\section{Appendix 5}

\section{Impedances and Substitution Corrections}

Ingles has studied the general impedance relationships in TV's and the effects of changing TE's.[9] However the following alternative approach, with its explicit treatment of phase defect angles, was developed at NBS. It has been used very effectively for several purposes, and so is given here. It is analogous to a much earlier unpublished NBS treatment of shunted TE'S for current measurements.

A. Impedance of a TVC

A TVC set with well-defined ac-dc characteristics (independent of the surroundings) generally consists of a shielded TE in series with one of a group of three-terminal shielded resistors, as shown in fig. 1 of [2]. As shown in section VI of [2], the ac-dc difference of a combination as a TVC is, in the present notation, $d_{v}=d_{t}+d_{c}$, where $d_{t}=\left(z_{t}-R_{t}\right) / R_{t}$ and $d_{c}=\left(I_{h a}-I_{h d}\right) / I_{h d}$. In these expressions, $Z_{t}=V_{i} / I_{h}$, and is the magnitude of the impedance of the combination, with an applied rms voltage $V_{i}$ and $a$ resultant heater current $I_{h}$. $I_{h a}$ and $I_{h d}$ are the magnitudes of the ac and dc currents required to produce the same output emf.

This is a very general equation, applicable for any series resistor and $\mathrm{TE}$ combination. To evaluate the component impedances we may write as an identity

$$
\bar{z}_{t}=\left(\bar{v}_{i}-\bar{v}_{h}\right) / \bar{I}_{h}+\bar{v}_{h} / \bar{I}_{h}=\bar{z}_{s}+\bar{z}_{h}
$$

where $\bar{v}_{h}$ is the voltage across the heater. The bars signify either phasors or complex numbers, and their absence signifies magnitudes. 
We may also write

$$
\bar{z}_{s} / R_{s}=1+a+j b \text { and } \bar{z}_{h} / R_{h}=1+c+j e
$$

with $a, b, c$ and e each much less than unity for a useful TVC. $R_{s}$ and $R_{h}$ are the dc values. Then

$$
\frac{\bar{z}_{t}}{R_{t}}=\frac{1+a+j b+p(1+c+j e)}{1+p}=1+g+j h
$$

where $p=R_{h} / R_{s}, g=(a+p c) /(1+p)$ and $h=(b+p e) /(1+p)$. Note that $h=\tan \alpha_{t}=\alpha_{t}$ the phase defect angle in radians, closely enough, since $g$ and $h$ are very small. Then to a sufficient approximation

$$
\frac{z_{t}}{R_{t}}=\left[1+2 g+h^{2}\right]^{1 / 2}=1+g+\frac{h^{2}}{2}
$$

and

$$
d_{t}=\frac{z_{t}}{R_{t}}-1=8+\frac{h^{2}}{2}
$$

When $p \leq 1, h^{2} / 2<1 \times 10^{-6}$ if $b$ and e are each less than 1 mrad, so that the phase angle term is negligible. In this case

$$
d_{t}=g, \quad d_{s}=a, \text { and } d_{h}=c
$$

where $d_{s}=\left(z_{s} / R_{s}\right)-1, d_{h}=\left(z_{h} / R_{h}\right)-1$. 


\section{B. Substitution Corrections (See Section IX)}

Consider two different $T E^{\prime} s$ of the same nominal $R_{h}$, each with a known $d_{C}$ as a current converter, and a known $d_{v h}$ as a voltage converter (with no series resistor). The difference in $d_{V}$ of a TVC range with the same series resistor, is, if all phase angles are negligible,

$$
d_{v 2}-d_{v 1}=8_{2}-g_{1}+d_{c 2}-d_{c 1}=p\left(c_{2}-c_{1}\right) /(1+p)+d_{c 2}-d_{c 1} \text {. }
$$

But

$$
p /(1+p)=R_{h} / R_{t} \text { and } c_{2}-c_{1}=d_{h 2}-d_{h 1}=\left(d_{v h 2}-d_{c 2}\right)-\left(d_{v h 1}-d_{c 1}\right) \text {. }
$$

Then

$$
d_{v 2}-d_{v 1}=\frac{R_{h}}{R_{t}}\left(d_{v h 2}-d_{v h 1}\right)+\frac{R_{s}}{R_{t}}\left(d_{c 2}-d_{c 1}\right) \text {. }
$$

Thus the substitution correction for any range is expressed in terms of the directly measurable d's of the new and old TE's as current and voltage converters.

If the phase angles are not negligible a term, $h_{1}{ }^{2}-h_{2}{ }^{2}$, must be added. This reduces to

$$
h_{1}^{2}-h_{2}^{2}=\frac{p b\left(e_{2}-e_{1}\right)+2 p^{2}\left(e_{2}^{2}-e_{1}^{2}\right)}{(1+p)^{2}}
$$

Thus it is zero if $e_{2}=e_{1}$.

If $b=1 \times 10^{-3}=e_{1}$ and $e_{2}=2 \times 10^{-3}$ (as rather extreme examples) this added phase angle correction is less than 1 ppm even at $p=1$ (low vollage 
range). It is much smaller for higher ranges, even if $b$ is larger (as it may be because of capacitive currents).

At NBS a commercial impedance comparator has been very convenient for comparing phase angles to better than $1 \mathrm{mrad}$ up to $100 \mathrm{kHz}$. It is used to screen the series resistors and TE heaters to make sure that their phase angles are negligible.

\section{Interchanged Series Resistors}

As explained in section $\mathrm{V}$, two nearly identical $30 \mathrm{~V}$ 3-terminal resistors, $R_{1}$ and $R_{2}$, of negligible phase angles, in separate coaxial shields, are connected in series with two nearly identical TE's. The resulting TVC's are compared, to evaluate $d_{c 1}-d_{c 2}$ of the TE's as current converters. Then the resistors are interchanged and the comparison is repeated to essentially eliminate inequalities in the magnitudes of the reactances of the resistors. For the first measurement, with $R_{1}$ in series with $T E_{1}$ and $R_{2}$ in series with $T E_{2}$,

$$
d_{T}-d_{S}=\Delta_{1}=\frac{a_{1}+p c_{1}-\left(a_{2}+p c_{2}\right)}{1+p}+d_{c 1}-d_{c 2}
$$

For the second measurement, with $R_{1}$ and $R_{2}$ interchanged

$$
\begin{aligned}
& \Delta_{2}=\frac{a_{2}+p c_{1}-\left(a_{1}+p c_{2}\right)}{1+p}+d_{c 1}-d_{c 2} \\
& \frac{\Delta_{1}+\Delta_{2}}{2}=\frac{R_{h}}{R_{t}}\left(c_{1}-c_{2}\right)+d_{c 1}-d_{c 2} \approx d_{c 1}-d_{c 2} \text {, closely enough, }
\end{aligned}
$$

since

$$
\frac{p}{1+p}=\frac{R_{h}}{R_{t}}=\frac{1}{60} \quad \text { for the usual } 0.5 \mathrm{~V} T E^{\prime} \text {. }
$$


A more extended treatment, including the effects of phase angles, leads to added terms in $b_{1}, b_{2}, e_{1}$ and $e_{2}$, similar to those given in appendix $5 B$. They are negligible if they do not exceed the limits given there.

\section{AC-DC Difference of Series Resistor}

For a given range of a TVC, from the equations of appendix 5A, with all phase angles negligible,

$$
d_{v}=d_{t}+d_{c}=\frac{d_{s}+p d_{h}}{1+p}+d_{c}
$$

Thus

$$
d_{s}=(1+p)\left(d_{v}-d_{c}\right)-p d_{h}=\frac{R_{t}}{R_{s}}\left(d_{v}-d_{c}\right)-\frac{R_{h}}{R_{s}} d_{h}
$$

since $d_{h}=d_{v h}-d_{c}$, where $d_{v h}$ is the ac-dc difference of the TE as a TVC.

\section{E. Effect of a Small Phase Difference Between the Paralleled Impedances in the Current Build Up}

The current through the single higher-range TE in the build-up is the phasor sum of the currents through the two paralleled lower-range TEs. It will differ from the sum of the magnitudes of the two currents, $I_{1}$ and $I_{2}$, if the two are not in phase.

For simplicity let $\bar{I}_{1}=I_{1} \angle 0, \bar{I}_{2}=I_{2} \angle \theta=I_{1} \angle \theta$ with $\theta<<1$ (in radians). The bar signifies a phasor quantity, and its absence a scalar. Since $I_{q}$ is the reference,

$$
\begin{aligned}
\bar{I} & =\bar{I}_{1}+\bar{I}_{2}=I_{1} \angle 0+I_{1} \angle \theta=I_{1}+I_{1}(\cos \theta+j \sin \theta) \\
& =I_{1}\left[\left(2+\theta^{2} / 2\right)+j \theta\right] \text { (neglecting higher order terms in } \theta \text { ) }
\end{aligned}
$$


From this and by continuing to neglect terms in $\theta^{4}$ and higher

$$
I=2 I_{1}\left(1-\theta^{2} / 8\right)
$$

The proportional difference is

$$
\varepsilon=\left(2 I_{1}-I\right) / I=\theta^{2} / 8 \text {. }
$$

If $\varepsilon=3 \times 10^{-7}, \theta=0.1 \times 10^{-3}$ radians. Since the two impedances, $\mathrm{Z}$, and $\mathrm{Z}_{2}$, are in parallel,

$$
\bar{I}_{1} \bar{Z}_{1}=I_{1} \angle O Z_{1} \angle 0=\bar{I}_{2} \bar{Z}_{2}
$$

This will be true only if $z_{2}=z_{1} \angle-\theta$. Thus the phase difference between $z_{1}$ and $z_{2}$ must be less than $1 \times 10^{-3}$ rad to keep the phase error less than about $0.3 \mathrm{ppm}$. 


\section{Appendix 6}

\section{RECOMMENDATION}

of the Working Group on the Statement of Uncertainties presented to Comite International des Poids et Mesures

Assignment of experimental uncertainties

\section{RECOMMENDATION INC-1 (1980)}

1. The uncertainty in the result of a measurement generally consists of several components which may be grouped into two categories according to the way in which their numerical value is estimated:

A - those which are evaluated by statistical methods,

B - those which are evaluated by other means.

There is not always a simple correspondence between the classification into categories A or B and the previously used classification into "random" and "systematic" uncertainties. The term "systematic uncertainty" can be misleading and should be avoided.

Any detailed report of the uncertainty should consist of a complete list of the components, specifying for each the method used to obtain its numerical value.

2. The components in category $A$ are characterized by the estimated variances, $s_{i}^{2}$, (or the estimated "standard deviations" $s_{i}$ ) and the number of degrees of freedom, $v_{i}$. Where appropriate, the estimated covariances should be given.

3. The components in category B should be characterized by quantities $u_{j}{ }^{2}$, which may be considered as approximations to the corresponding variances, the existence of which is assumed. The quantities $u_{j}{ }^{2}$ may be treated like 
variances and the quantities $u_{j}$ like standard deviations. Where appropriate, the covariances should be treated in a similar way.

4. The combined uncertainty should be characterized by the numerical value obtained by applying the usual method for the combination of variances. The combined uncertainty and its components should be expressed in the form of "standard deviations".

5. If, for particular applications, it is necessary to multiply the combined uncertainty by a factor to obtain an overall uncertainty, the multiplying factor used must always be stated. 


\title{
Thermal Converters for Audio-Frequency Voltage Measurements of High Accuracy
}

\author{
F. L. HERMACH, FELLOW, IEEE, AND E. S. WILLIAMS
}

Abstract-The ac-dc differences of a reference group of thermoelements have been evaluated at audio frequencies to a few parts per million (ppm) at currents from 5 to $20 \mathrm{~mA}$. A technique for comparing the ac-dc differences of two thermoelements with an uncertainty of about $2 \mathrm{ppm}$ has been developed. Two $5 \mathrm{~mA}$ thermoelements are used with a plug-in set of resistors of computable reactances to form thermal voltage converters for voltage measurements. With this same technique adjacent ranges of these converters can be compared to step up from 0.5 to $500 \mathrm{~V}$ to better than $10 \mathrm{ppm}$.

\section{INTRODUCTION}

I PRINCIPLE, the current balances which have been developed to determine the ampere in absolute measure with direct current could also be used with alternating current. However, the most stable electrical standards are still the dc standard cell, and $1 \mathrm{ohm}$ resistors, which are not usually designed for ac use. Thus, ac measurements of current and voltage have, in practice, been based on rms ac-dc transfer standards or comparators. For many decades these were electrodynamic or electrostatic instruments, designed to have the same torque constant with either alternating or direct current (or voltage) applied, making it easier to obtain a wider frequency range than with instruments like the current balance which must have a computable torque constant. More recently, electrothermic instruments such as thermocouple transfer standards and bolometer or thermistor bridges have been used. All of these instruments have been highly developed in the national laboratories of several countries to make ac measurements at audio frequencies to about $100 \mathrm{ppm}$ in combination with de standards. The combination possesses a number of advantages over ac potentiometers (which must also include a transfer instrument), and over electronic and other squaring circuits (which must have exact squarelaw characteristics in two quadrants).

Recent advances in operational amplifiers and inductive voltage dividers have made possible wide-range ac power sources with linearity and day-to-day stability better than $100 \mathrm{ppm}$. This implies a need for better accuracy in basic ac-dc transfer standards.

At this accuracy, conventional forms of electrodynamic transfer instruments are limited to power frequencies by the effects of inductive and capacitive re-

Manuscript received June 23,1966 . This paper was presented at the 1966 Conference on Precision Electromagnetic Measurements, Boulder, Colo. This work was supported in part by the Army Metrology and Calibration Center, Frankford Arsenal, and the Metrology Engineering Center, Bureau of Naval lleapons, Pomona, Calif.

The authors are with the Electrical Instruments Section, Electricity Division, National IBurenu of Standards, Gaithersburg, Md. actances in their coils, and electrostatic instruments have a serious minimum-voltage limitation because of their low torque-weight ratio. Both would require new means of sensing deflections to obtain precisions of 10 ppm or better. This could be done by measuring the reactance, which is a function of the deflection of the instrument. However, a major difficulty would remain - that of vibrations from external forces or from the cyclic variations of the ac driving torque. Experience has shown that it is of ten difficult to control spurious torques caused by minor mechanical resonances. It is possible that floating the moving system in a liquid of the same density would minimize these difficulties. However, it has seemed to the authors that improved thermal converters (thermocouple instruments) offer better promise of obtaining an ac-dc transfer accuracy of $10 \mathrm{ppm}$ or better, and considerable effort has been concentrated on them over the past five years. This paper may be considered as a progress report describing the present group of reference thermoelements and coaxial thermal voltage converters, and the techniques and equipment which have been developed to compare them to a few ppm.

\section{Thermal Converters}

A thermoelement (TE), defined as the simplest type of thermal converter [1], consists of a heater and thermocouple. In its usual form, the heater is a short, straight wire suspended between two supporting leadin wires in an evacuated glass bulb. The hot junction of a thermocouple is fastened to the midpoint of the heater, and is electrically insulated from it with a small bead. The thermocouple EMF (about 5 to $10 \mathrm{mV}$ for a conventional TE at rated current) ${ }^{1}$ is then a measure of the heater current. For voltage measurements, a resistor is connected in series with the heater to form a thermal voltage converter [1].

At the National Bureau of Standards, fourteen 'T Es form the present reference group for ac-dc difference. Twelve of them (made by four different manufacturers) are of conventional design but with heaters of Evinohm or Karma (modified nickel-chromium alloys), which have low thermoelectric effects. ${ }^{2}$ They were chosen from

1 The international system (SI) of unitu with its symbols is uwel in this paper. Because of their freguent iscurrence, the wersd thermorle. ment is abbreviated as ' $\mathrm{TF}$, and thermill voltage crullerter as $11 \mathrm{C}^{\circ}$ in this paper.

${ }^{2}$ Evanohm and Karma are registered trollemarks of the Willus B. Driver Company and the I)river-IIarri, Companyy, revincliorl, (See $\Lambda$ cknowledgments.) 

larger groups on the basis of low dc reversal differences and high bead resistances, and are all mounted in one aluminum enclosure lined with foamed plastic for thermal insulation. Each is supported by its heater leads, which are soldered directly to binding posts mounted on the low-loss phenolic top plate of the enclosure, and its thermocouple output leads, which are soldered to two-pin connectors. The other two thermal current converters are of a radically different design having many thermocouples ( 40 or more) attached to a bifilar (doubled-back) heater [2]. They were obtained from their inventor, F. J. Wilkins of the National Physical Laboratory in England.

The fourteen TEs are identified in Table I, along wilh certain pertinent characteristics, as well as the acde differences assigned to them at audio frequencies as a result of the tests to be described.

TABLE I

ReFEREnce Thermolelements

\begin{tabular}{|c|c|c|c|c|c|c|c|}
\hline \multirow{3}{*}{$\begin{array}{l}\text { Identi- } \\
\text { fication }\end{array}$} & \multicolumn{2}{|c|}{ Rated } & \multirow{3}{*}{$\begin{array}{c}\text { T) } \\
\text { re- } \\
\text { versalt } \\
\text { ppm }\end{array}$} & \multirow{3}{*}{$\begin{array}{c}\text { Approx. } \\
\text { heater } \\
\text { length } \\
\mathrm{mm}\end{array}$} & \multirow{3}{*}{$\begin{array}{c}\text { Bead } \\
\text { resis- } \\
\text { tance } \\
G \Omega\end{array}$} & \multirow{2}{*}{\multicolumn{2}{|c|}{$\begin{array}{l}\text { Ad-dc differ- } \\
\text { ence (ppm) }\end{array}$}} \\
\hline & \multirow{2}{*}{$\begin{array}{l}\text { Cur- } \\
\text { rent } \\
m A\end{array}$} & \multirow{2}{*}{$\begin{array}{c}\mathrm{EMF} \\
\mathrm{mV}\end{array}$} & & & & & \\
\hline & & & & & & $20 \mathrm{~Hz}$ & $2 \mathrm{kHz} \|$ \\
\hline$\therefore$ I'L $14^{*}$ & 20 & 25 & $<10$ & - & $>1$ & 0 & 0 \\
\hline NI'L 15* & 20 & 25 & $<10$ & - & $>1$ & 0 & 0 \\
\hline$\triangle 20 \mathrm{E} 3$ & 20 & 5 & 40 & 5 & $>1$ & 0 & 0 \\
\hline 120E6 & 20 & 5 & 60 & 5 & $>1$ & 0 & -1 \\
\hline A 10E:18 & 10 & 8 & 130 & 5 & 0.3 & +6 & +1 \\
\hline A10E 19 & 10 & 8 & 160 & 5 & 0.5 & 0 & -1 \\
\hline W10E 1 & 10 & 7 & 200 & 8 & 0.6 & +1 & +3 \\
\hline II 10E.2 & 10 & 7 & $<10$ & 8 & 0.8 & -2 & 0 \\
\hline $1310 E 45$ & 10 & 8 & 160 & 4 & $>1$ & -8 & -2 \\
\hline †5E 8 & 5 & 7 & 10 & 5 & 0.7 & +1 & +1 \\
\hline . $5 \mathrm{ES9}$ & 5 & 6 & 40 & 5 & 0.6 & 0 & -1 \\
\hline F5lig & 5 & 4 & 20 & 5 & 0.5 & -3 & -2 \\
\hline F.5k13 & 5 & 4 & 260 & 5 & 0.5 & -2 & 0 \\
\hline $135 \mathrm{E} 77$ & 5 & 8 & 110 & 6 & $>1$ & +6 & 0 \\
\hline
\end{tabular}

* These can be used at much higher currents.

$\dagger \Delta I / I$ for the same EMF.

$\ddagger$ At $2 \mathrm{kHz}$ and rated $I$.

I| Same value assigned at $20 \mathrm{kHz}$.

\section{Thermal Voltage Converters (TVCs)}

Each of a new set of TVCs developed at NBS for this work consists of one or more cylindrical metal-film resistors in a coaxial metal cylinder. The resistors can be connected in series with one of two $5 \mathrm{~mA}$ TEs mounted in separate cylinders so that the output EMF is then a measure of the input voltage. Like an earlier set developed at NBS [3], the effect of the reactance on the frequency response can be computed at least roughly from the simple geometry. Adjacent ranges of these TVCs may be compared with greater certainty than those of the older design, which had integrally mounted TEs.

As shown in Fig. 1, a single two-watt resistor is used for each range below $200 \mathrm{~V}$. The higher-voltage resistor units have two or more resistors in larger cylinders, with two adjustable inner shields to control capacitance currents, as shown in Fig. 1, and have ports and baffles to permit the controlled flow of cooling air from the laboratory compressed-air supply.
Pertinent data on the TVCs, and the ac-dc differences assigned to each range are given in Table II. The TVCs are shown in Fig. 2.

\section{EMF COMPARATOR}

The ac-dc differences of a transfer instrument may be defined as

$$
\delta=\left(X_{a}-X_{d}\right) / X_{d}
$$

where $X_{a}$ and $X_{d}$ are the magnitudes of the ac and dc quantities that are required to give the same response (such as deflection or output EMF) of the instrument. (Normally, the average for the two directions of the dc quantity gives the best measure of the dc reference.) This is a useful definition, for the ac quantity for a given response is then simply $X_{a}=X_{d}(1+\delta)$. As is well known, the instrument may be used as an ac-dc transfer standard simply by observing the response with the ac quantity applied, then measuring (with external standards) the average for the two dc quantities required to obtain the same response, thus avoiding many of the limitations of an ordinary instrument.

At the National Bureau of Standards, TEs are evaluated in pairs with the EMF comparator shown in Fig. 3, to determine the differences in their ac-dc transfer performance, i.e., $\delta_{1}-\delta_{2}$ as defined previously. TVCs are connected in parallel and evaluated in the same way. With the desired current or vol tage applied, $R_{1}$ and $R_{2}$ are adjusted in a preliminary balance to obtain a near null on the detector $D_{1}$. Then, in regular succession, alternating-, direct-, reversed direct-, and alternatingcurrents are applied to the TEs at nearly equal time in tervals and without changing $R_{1}$ or $R_{2}$. In each case, the current is adjusted to obtain the same output EMF, $E_{1}$ of $T E_{1}$, as indicated by an auxiliary Lindeck potentiometer $\left(P\right.$ with its detector $\left.D_{2}\right)$, and the resulting deflections of $D_{1}$ are observed. Then, as shown in Appendix $I$, the relative ac-dc difference is

$$
\delta_{1}-\delta_{2}=s\left(D_{a}-D_{d}\right)
$$

where $D_{a}$ and $D_{d}$ are the averages of $D_{1}$ with alternating and direct currents applied, respectively. The sensitivity factor $s$ can be determined in one of several ways, as indicated in the Appendix, such as by changing the current in the heater of $T_{2}$ by a small known amount and observing the resulting deflection of $D_{1}$.

The secpuence of readings, nearly equally spaced in time, greatly reduces errors from drifts, icmperature changes, etc., in either TE and from either constint or slowly changing thermal E.Mlis in the comparator. The comparator accommodates any reasonalule range of thermocouple EMFs, which need not be erpual, and the small currents (up to $20 \mu \mathrm{A}$ ) in the thermocouple circun do not affect the measured valures.

With this circuit, small fluctuations in the supply. (heater) current do not appreciably change the defler. tion if the two 'T Es have reatsonably equal proportional changes in EMF $(\Delta E / E)$, with a given clange in lecoler current and reasonably equal time constants. Although 

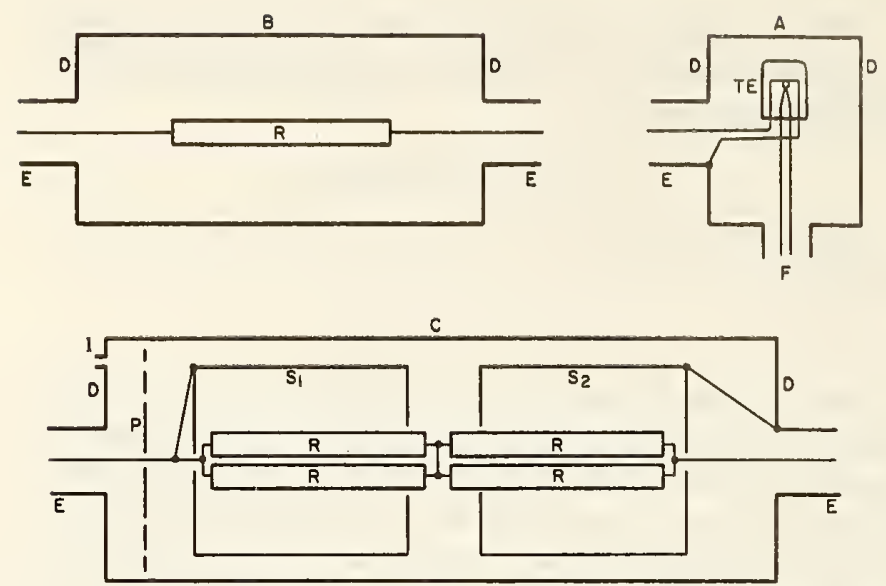

Fig. 1. Essentials of thermal voltage converters (construction details not shown).

A Thermoelement unit, with $5 \mathrm{~mA} 100 \Omega \mathrm{TE}$, for $0.5 \mathrm{~V}$, in brass cylinder, $4 \mathrm{~cm}$ long and $5.1 \mathrm{~cm}$ diameter, with brass end plate D.

B Low-voltage resistor unit ( 1 to $100 \mathrm{~V}$ ) with one 2-watt metal film resistor $\mathrm{R}$ in brass cylinder $11 \mathrm{~cm}$ long and $5.1 \mathrm{~cm}$ diameter.

C High-voltage resistor unit $(200-500 \mathrm{~V})$ with multiple 2-watt metal-film resistors, in brass cylinder $18 \mathrm{~cm}$ long and $6.4 \mathrm{~cm}$ diameter with brass end plate D. Air inlet at I, outlet holes at other end (not shown).

$\mathrm{S}_{1}, \mathrm{~S}_{2}$ Inner brass shields $5.5 \mathrm{~cm}$ long, $5.1 \mathrm{~cm}$ diameter, connected as shown, with no end plates. $S_{2}$ can be moved parallel to axis and locked in place.

$P$ Non-conducting air-baffle plate with holes.

E Coaxial connector GR874.

F Two-pin output connector AN10SL.

it would be difficult to calculate the resultant "stabilization factor" of the circuit, in practice, fluctuations are much less than those observed when the output EMF of each $\mathrm{TE}$ is balanced against a constant voltage source, as in earlier comparators [4]. Thus, with this circuit and technique, TEs may be intercompared with an imprecision which is much less than the fluctuations of the supplies used.

$R_{1}$ consists of ten $100 \Omega$ resistors mounted on an enclosed-contact silver-alloy switch whose thermal EMFs are much less than $0.1 \mu \mathrm{V}$ when the wiper is stationary. $R_{2}$ is a $100 \Omega$, 10-turn helical resistor of manganin, with a manganin wiper to minimize thermal EMFs. Detector $D_{1}$ consists of a commercially available primary galvanometer, incorporating photocells in an adjustable negative-feedback circuit, which serves as a current amplifier to a secondary galvanometer. The scale factor of the combination is about $10 \mathrm{pA} / \mathrm{mm}$, and the input resistance (which is dependent on the negative feedback) ranges from 200 to $3000 \Omega$. The primary galvanometer is liquid filled to reduce disturbances from mechanical shocks and vibrations and, with its period of two seconds, is an excellent mechanical lowpass filter. A similar pair of galvanometers is used for $D_{2}$. Both secondary light beams appear on one scale so that the observer may quickly read the deflection of $D_{1}$ if it is stationary and if $D_{2}$ is in the desired range. (Residual
TABLE II

NBS "MOdel F" Thermal Voltage Converters

\begin{tabular}{|c|c|c|c|c|c|c|}
\hline \multirow{2}{*}{ Range $\mathrm{V}$} & \multicolumn{2}{|c|}{ Resistors } & \multirow{2}{*}{$\begin{array}{c}\text { Total } \\
k \Omega \dagger\end{array}$} & \multicolumn{3}{|c|}{$\delta_{\mathrm{r}}(\mathrm{ppm}) \ddagger$} \\
\hline & No. & $\begin{array}{c}k \Omega \\
\text { (each) }\end{array}$ & & $2 \mathrm{kHz}$ & $20 \mathrm{kHz}$ & $50 \mathrm{kHz}$ \\
\hline $\begin{array}{l}0.5-A^{*} \\
0.5-B^{*} \\
1 \\
2 \\
3 \\
5 \\
10 \\
20 \\
30 \\
50 \\
100 \\
200 \\
300 \\
500\end{array}$ & \begin{tabular}{|c} 
none \\
none \\
1 \\
1 \\
1 \\
1 \\
1 \\
1 \\
1 \\
1 \\
1 \\
4 \\
6 \\
8
\end{tabular} & $\begin{array}{c}0.1 \\
0.3 \\
0.5 \\
0.9 \\
1.9 \\
3.9 \\
6 \\
10 \\
20 \\
40 \\
90 \\
200\end{array}$ & $\begin{array}{r}0.1 \\
0.1 \\
0.2 \\
0.4 \\
0.6 \\
1.0 \\
2.0 \\
4.0 \\
6.1 \\
10 \\
20 \\
40 \\
60 \\
100\end{array}$ & $\begin{array}{r}+1 \\
-4 \\
-2 \\
-3 \\
-1 \\
-1 \\
0 \\
0 \\
+1 \\
+1 \\
+1 \\
+1 \\
+1 \\
+1\end{array}$ & $\begin{array}{r}+1 \\
-2 \\
0 \\
-2 \\
0 \\
0 \\
+1 \\
+1 \\
+1 \\
0 \\
0 \\
0 \\
0 \\
-6\end{array}$ & $\begin{array}{l}+15 \\
+11 \\
+11 \\
+3 \\
+3 \\
=1 \\
=1 \\
=1 \\
=2 \\
-4 \\
-7 \\
-7 \\
-3 \\
+13\end{array}$ \\
\hline
\end{tabular}

* TEs $\mathrm{A}$ and $\mathrm{B}$ as TVCs, without series resistors.

$\dagger$ Including $0.5 \mathrm{~V}$ TE. Resistors connected in series-parallel for higher ranges.

$\ddagger \delta_{\mathrm{r}}$ is the impedance error only (see text) computed from Table III with either TE of pair \#1. A similar table with the \#2 pair differs from it by not more than $1 \mathrm{ppm}$ at 2 and $20 \mathrm{kHz}$ except at $0.5 \mathrm{~V}$ range.

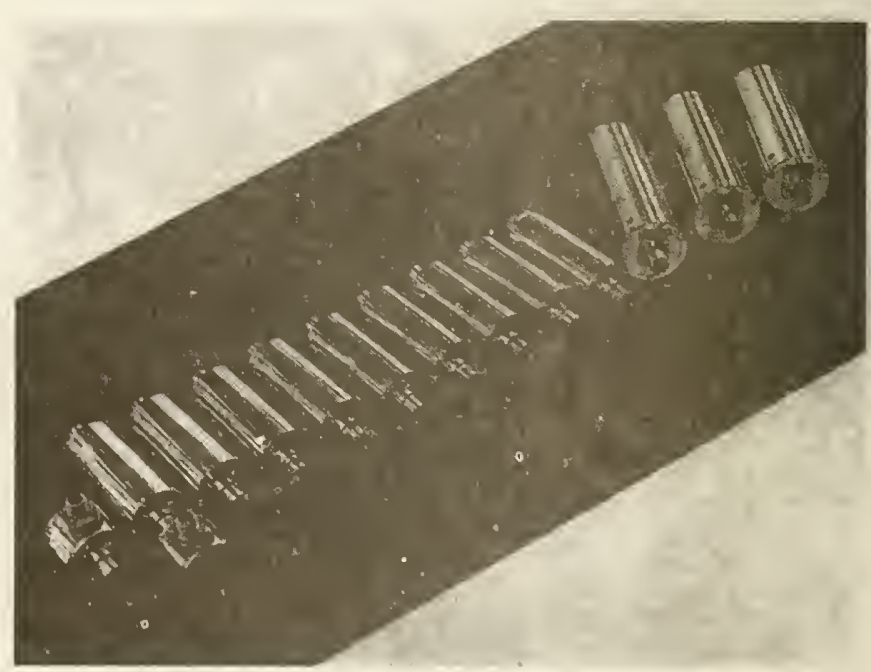

Fig. 2. Thermal voltage converters.

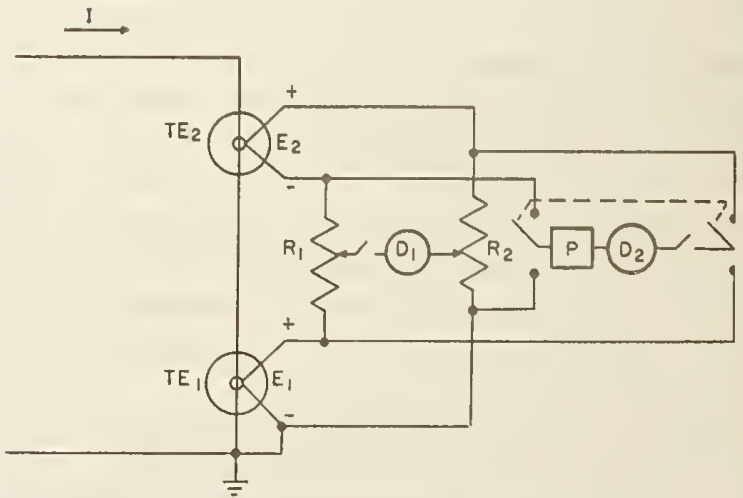

Fig. 3. Essentials of EMF comparator. 
deflections of $D_{2}$ are less critical if the TEs are well matched.)

All of the components of the comparator except the detectors are mounted in a metal box behind a metal panel to provide electrostatic shielding, and the box is lined with foamed plastic to reduce temperature fluctuations. The metal cases of the primary galvanometers are connected to this box by the shields of two-conductor cables.

With $R_{1}$ and $R_{2}$ at their midpoints and each of the thermocouple inputs to the EMF comparator shortcircuited, peak-to-peak fluctuations in the secondary galvanometer of $D_{1}$ in periods of 10 seconds amount to 1 $\mathrm{mm}$ at the gain setting normally used. With $7 \mathrm{mV}$ at each thermocouple input, this corresponds to a value of $\delta_{1}-\delta_{2}$ of $2 \mathrm{ppm}$. This is only about four times the calculated Johnson-noise value for this circuit.

A comparison of two TEs (or TVCs) usually consists of four or more determinations of $\delta_{1}-\delta_{2}$ by the method described. Three times the standard error (standard deviation of the average) computed from a number of typical series of such measurements is about $2 \mathrm{ppm}$. This is a reasonable measure of the uncertainty in the comparator. Comparisons of various pairs taken from three TEs, with the TEs interchanged in the comparator and with the EMF of first one TE and then the other held constant in each sequence, have disclosed no systematic error as large as $1 \mathrm{ppm} .{ }^{3}$ These measurements were made with commercially available dc and ac voltage sources, having peak-to-peak instabilities, observed with a TVC in 10-second intervals, of about 10 and $20 \mathrm{ppm}$, respectively, at $2 \mathrm{kHz}$.

A TVC can be used for ac measurements with appropriate dc standards by balancing its output EMF against an adjustable stable voltage source such as the potentiometer $P$. To reduce errors from drifts, a similar sequence of ac and dc readings can be used, with the setting of $P$ fixed, and the direct voltage $V_{d}$ preset and accurately known. Then

$$
V_{a}=V_{d}\left[1+\delta+s\left(D_{a}-D_{d}\right)\right]
$$

where $s$, the sensitivity factor, is determined as before.

\section{Comparisons of TEs}

The fourteen reference TEs were compared, using sinusoidal currents at frequencies of $20 \mathrm{~Hz}$, and 2 and $20 \mathrm{kHz}$. All of these measurements (as well as the comparisons of TVCs) were made in a shielded room to reduce electromagnetic interference, which is particularly troublesome with TEs because of their high sensitivity and very wide frequency range. The step down from onc current to the next was made by connecting

${ }^{3}$ If the net dc reversal difference of the two 'TEs is more than 300) ppm, an error is introduced by nonlinearities in $D_{1}$. This can be minimized by taking determinations with first one and then the other 'IF: E.III held corstant. the heaters of two low-current TEs, $a$ and $b$, in parallel and their thermocouples in series aiding, and considering the combination as a single $\mathrm{TE}$ connected in the $\mathrm{TE}_{1}$ position of Fig. $3 .^{4}$ As shown in Appendix II, if the TEs are reasonably well matched, the ac-dc difference of the parallel combination is simply $\delta_{p}=\left(\delta_{a}+\delta_{b}\right) / 2$ to better than $1 \mathrm{ppm}$. From the test, $\delta_{p}-\delta_{2}=K_{1}$ where $\delta_{2}$ is the ac-dc difference of the high range TE in position 2 , and $K_{1}=s\left(D_{a}-D_{d}\right)$. The two low-current TEs are then connected in series and compared to determine $K_{2}=\delta_{a}-\delta_{b}$. Thus, $\delta_{a}=K_{1}+\delta_{2}+K_{2} / 2$ and $\delta_{b}=K_{1}+\delta_{2}$ $-K_{2} / 2$.

The network of comparisons at $2 \mathrm{kHz}$ and the results are shown in Fig. 4. Here the circles identify individual TEs and the rectangles represent a pair in parallel. The arrows indicate comparisons, and the numbers on them are the values of the relative ac-dc differences in $\mathrm{ppm}$; i.e., the ac-dc difference of the TE at the point of the arrow is greater or less than that at the tail by the amount shown. These tests have extended over a period of three years during the development of this project; in many cases, each number is the average of two or more tests, each consisting of four or more sequences of ac-dc readings already described. ${ }^{5}$

As indicated by Wilkins, the ac-dc difference of the two NPL TEs should, theoretically, be less than $1 \mathrm{ppm}$. In tests at NBS at $20 \mathrm{~mA}$, the two agreed to better than $0.2 \mathrm{ppm}$ with a $3 \sigma$ imprecision of $0.3 \mathrm{ppm} .^{6}$ Thus, it is reasonable as well as convenient to start with an assignment of zero ppm to the ac-dc differences of each. Then, following principles given by Youden [5] in weighting the various paths in a network, it is possible to calculate the ac-dc differences of the other TEs. The resulting values are given to the nearest ppm in each circle and in Table I.

The average for all of the conventional TEs is only $\mathbf{- 0 . 3} \mathrm{ppm}$. Thus, these simple TEs agree with the more complicated multijunction converters very closely, and essentially the same values would have been obtained for each TE on the alternative basis of assigning zcro to the average of the entire group.

A complete but less redundant chain of measure. ments was carried out at $20 \mathrm{~Hz}$ to evaluate low-frequency errors of each 'TE. The precision was less at that frequency because of much larger instability in the ac source. The results of the values assigned on the basis of zero error for the NPL TIS are given in Table I

Not enough TEs were intercompared at $20 \mathrm{kHz}$ (n) assign independent values to each, because acode dif.

\footnotetext{
4 To minimize certain aberrations discuserl in torluon 1.8 , or it. tors of at least five times the heiter resistance were added tw ear h in the heaters before paralleling them.

o Becallse ineasurements were mide over all extruded time in developing program, the networh in llot symunctrioul.

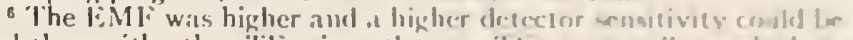
used thatl with other llos silice the 1 wo Tl:a ware well matherl. on that all extraturdinarily high precision was allonum
} 


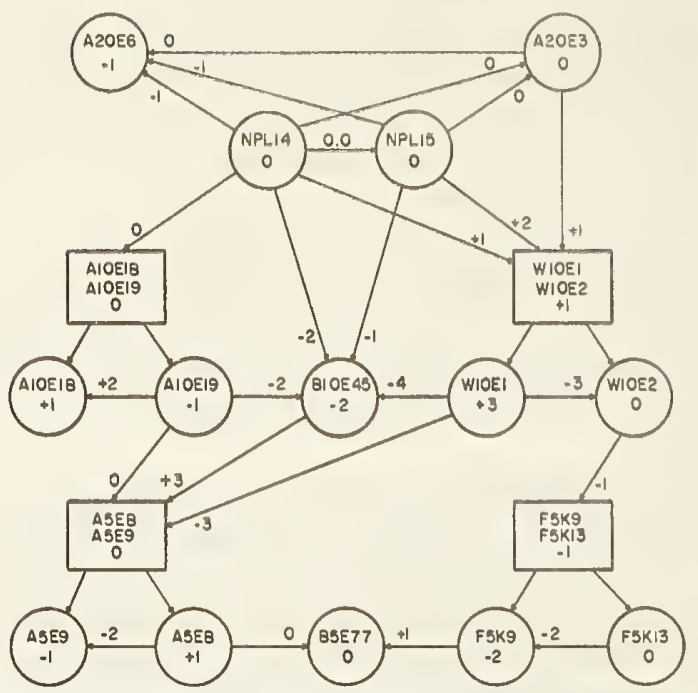

Fig. 4. Intercomparisons of NBS TEs, and assigned ac-dc differences in ppm at $2 \mathrm{kHz}$.

ferences from all known sources [3] should be the same as at $2 \mathrm{kHz}$ within $1 \mathrm{ppm}$. No measured values at the two frequencies differed by more than $2 \mathrm{ppm}$. Thus, the same values were assigned to each $\mathrm{TE}$ at the higher frequency.

\section{Comparisons of TVCs}

The TVCs were designed so that adjacent ranges could be compared to step up and down the voltage scale. Their nearly identical $5 \mathrm{~mA}$ TEs were each first compared with one of the reference group of TEs as current converters, i.e., with the heaters connected in series. They were then paralleled through a " $T$ " connector and compared with each other as voltage converters (basic range $0.5 \mathrm{~V}$ ). The lowest resistor (see Table 2) was then connected in series with one TE, say $\mathrm{TE}_{a}$, to make a one-volt TVC. This was connected in parallel with the other, $\mathrm{TE}_{b}$, and compared at 0.5 volt. A twovolt TVC was then formed with the next resistor and $\mathrm{TE}_{b}$, and compared with the one-volt TVC at $1 \mathrm{~V}$. This process was continued until the highest range was reached. If the ac-dc difference of each combination is independent of the voltage level, the ac-dc difference of any range may be found in terms of one as a reference. This requirement is easily met if the TEs have no signifcant errors, for the reactance errors of each resistor unit. should be independent of voltage. The validity of this assumption itself can easily be verified by making each comparison at two voltage levels.

If we define $Z_{t}=V_{i} / I_{0}$ for a TVC, where $V_{i}$ is the magnitude of the applied voltage and $I_{o}$ is the magnitude of the current through the TE, then, from the definition for ac-dc difference, applied to a TVC,

$$
\delta_{v}=\frac{V_{\text {in }}-V_{\text {id }}}{V_{\text {id }}}=\frac{I_{o a} Z_{t}-I_{o d} R_{t}}{I_{o d} R_{t}}
$$

where the subscripts $a$ and $d$ refer to alternating and direct current (or voltage). Since $I_{o a}$ and $I_{o d}$ produce equal output EMFs, $I_{o a}=I_{o d}\left(1+\delta_{o}\right)$. If we let $Z_{t}$ $=R_{t}\left(1+\delta_{r}\right)$, we get, if second-order terms are negligible, $\delta_{v}=\delta_{r}+\delta_{0}$. Thus, $\delta_{v}$ can be determined if $\delta_{r}$ can be deduced from the comparisons of adjacent ranges, since $\delta_{0}$ can be evaluated in terms of the reference group of TEs.

Two complete sets of adjacent-range comparisons from 0.5 to $500 \mathrm{~V}$ were carried out at frequencies of 2 , 20 , and $50 \mathrm{kHz}$, with two different pairs of $5 \mathrm{~mA}$ TEs. These TEs had $80 \mathrm{Ni} 20 \mathrm{Cr}$ heaters but were otherwise similar to those in the reference group. Measured values of $\delta$ as current converters were -1 or $-2 \mathrm{ppm}$, except at $20 \mathrm{~Hz}$ where they ranged from -6 to $-9 \mathrm{ppm} .{ }^{7}$ The results of the adjacent-range tests given in Table III showed excellent precision. In addition, the inter-range differences were less than $2 \mathrm{ppm}$ through $20 \mathrm{kHz}$, except for the lowest and highest ranges. The differences of a few ppm observed on the lowest ranges were rather puzzling and will be discussed in Section IX. They were essentially independent of frequency through 20 $\mathrm{kHz}$ but were negligible above the 1 volt range.

TABLE III

Comparisons of Thermal Voltage Converters

\begin{tabular}{|c|c|c|c|c|c|c|c|c|}
\hline \multirow{3}{*}{ TVC 1} & \multirow{3}{*}{ TVC 2} & \multirow{3}{*}{$\begin{array}{c}\text { Voltage } \\
\mathrm{V}\end{array}$} & \multicolumn{6}{|c|}{$\delta_{1}-\delta_{2}$ in $\mathrm{ppm}$} \\
\hline & & & \multicolumn{2}{|c|}{$2 \mathrm{kHz}$} & \multicolumn{2}{|c|}{$20 \mathrm{kHz}$} & \multicolumn{2}{|c|}{$50 \mathrm{kHz}$} \\
\hline & & & $\# 1^{*}$ & $\# 2 \dagger$ & $\not 1$ & $\# 2$ & $\frac{\| 1}{\pi} 1$ & $\# 2$ \\
\hline $\begin{array}{r}\mathrm{A} \\
\mathrm{B} \\
1-\mathrm{B} \\
1-\mathrm{A} \\
2-\mathrm{B} \\
3-\mathrm{A} \\
5-\mathrm{B} \\
10-\mathrm{A} \\
20-\mathrm{B} \\
30-\mathrm{A} \\
50-\mathrm{B} \\
100-\mathrm{A} \\
200-\mathrm{B} \\
300-\mathrm{A} \\
500-\mathrm{B}\end{array}$ & $\begin{array}{r}\mathrm{B} \\
\mathrm{A} \\
\mathrm{A} \\
\mathrm{B} \\
1-\mathrm{A} \\
2-\mathrm{B} \\
3-\mathrm{A} \\
5-\mathrm{B} \\
10-\mathrm{A} \\
20-\mathrm{B} \\
30-\mathrm{A} \\
50-\mathrm{B} \\
100-\mathrm{A} \\
200-\mathrm{B} \\
300-\mathrm{A}\end{array}$ & $\begin{array}{r}0.5 \\
0.5 \\
0.5 \\
0.5 \\
1 \\
2 \\
3 \\
5 \\
10 \\
20 \\
30 \\
50 \\
100 \\
200 \\
300\end{array}$ & $\begin{array}{r}-3 \\
+2 \\
+2 \\
-1 \\
+2 \\
0 \\
+1 \\
0 \\
+1 \\
0 \\
0 \\
0 \\
0 \\
0\end{array}$ & -3 & $\begin{array}{r}-4 \\
0 \\
+2 \\
-2 \\
+2 \\
0 \\
+1 \\
0 \\
0 \\
-1 \\
0 \\
0 \\
0 \\
-6\end{array}$ & $\begin{array}{r}+2 \\
-3 \\
+1 \\
-1 \\
+1 \\
0 \\
0 \\
+1 \\
0 \\
0 \\
0 \\
-1 \\
0 \\
-5\end{array}$ & $\begin{array}{r}-4 \\
-2 \\
0 \\
-8 \\
0 \\
-4 \\
0 \\
0 \\
-1 \\
-2 \\
-3 \\
0 \\
+4 \\
+16\end{array}$ & $\begin{array}{r}-4 \\
+3 \\
-4 \\
-1 \\
0 \\
-2 \\
-1 \\
0 \\
0 \\
-2 \\
+4 \\
+6 \\
+8\end{array}$ \\
\hline
\end{tabular}

* Test \#1-With one pair of $5 \mathrm{~mA}, 100 \mathrm{ohm}$ TEs.

$\dagger$ Test \#2-With another pair of $5 \mathrm{~mA}, 100 \mathrm{ohm}$ TEs. These tests were made three months after those with pair $\# 1$.

Except for this, the results of the comparisons are in excellent agreement with computed values (see Section VIII), which show that $\delta_{r}$ should be less than $1 \mathrm{ppm}$ through the $100 \mathrm{~V}$ range. It is extremely unlikely that any single source or combination of sources of ac-dc difference should produce the same $\delta_{r}$ in each of these ranges. Thus, the authors believe that, from the concordance of theory and experiment, it is safe to assign zero $\mathrm{ppm}$ to the average of the values of $\delta_{r}$ from the $2 \mathrm{~V}$

7 'These IFs, which were the only' ones available at the time will the correct heiter resistince, will be replaced by others. 
through the $100 \mathrm{~V}$ ranges in establishing the values given in Table II from the comparisons of Table III.

The comparisons at $50 \mathrm{kHz}$ (which were less precise) were made to verify that ac-dc differences were not sharply dependent on frequency at $20 \mathrm{kHz}$. They indicate that excellent accuracy should be expected for most ranges at even higher frequencies.

A complete set of measurements at 75 percent of rated voltage was also made at $2 \mathrm{kHz}$ with TE pair $\# 1$ in order to investigate voltage coefficients. These results agreed with the rated-voltage measurements to 1 ppm, with no systematic difference.

A completely independent verification of the accuracy of these reference TVCs has come from some careful comparisons by Flach and Marzetta [8] of a "time-gate" peak ac-to-dc voltage comparator (designed by Marzetta) with an rms comparator which incorporated a TVC that had been calibrated with the reference set. In a long series of tests at 400 and $1000 \mathrm{~Hz}$, where the error in the comparison introduced by distortion of the ac source was less than $10 \mathrm{ppm}$, the two comparators agreed on the average to 4 and $8 \mathrm{ppm}$, respectively, at the two frequencies.

\section{Thermoelectric ERrors}

Hermach's treatment of the ac-dc errors arising from Thomson effects in the heater of a conventional TE [4] has been extended by Widdis [6] to include the effects of Peltier heating, and to correct for the heat abstracted by the thermocouple. Widdis' expression for the Thomson ac-dc difference, $\delta_{t}=\alpha^{2} \theta / 12 \rho k$, is the same as that found by Hermach for high-range TEs, where $\alpha, \rho$, and $k$ are, respectively, the Thomson coefficient, electrical resistivity, and thermal conductivity of the heater alloy, and $\theta$ is the midpoint temperature rise $\left(100^{\circ} \mathrm{C}\right.$ to $150^{\circ} \mathrm{C}$ in a conventional $\mathrm{TE}$ ). The error can thus be computed reasonably well if $\alpha$ can be determined. The Thomson heating of copper is reasonably well known [7], and is not much affected by moderate temperature elevations or by the cold working of ordinary handling. Thus, this metal can serve as a practical reference. The authors have calculated the Seebeck and the absolute Thomson coefficients of a number of pertinent alloys from careful measurements of the EMF vs. temperature of thermocouples formed from each of these alloys and copper. The results are shown in Table IV, along with the calculated ac-dc difference $\delta_{l}$ (using Widdis' formula) in a TE having midpoint heater temperature rise of $150^{\circ} \mathrm{C}$ (giving a $10 \mathrm{mV}$ output with a typical $\mathrm{NiCr}$ constantan thermocouple). With Evanohm or Karma, $\delta_{t}$ should not exceed $1 \mathrm{ppm}$. At an output of $5 \mathrm{mV}$ it should be less than $1 \mathrm{ppm}$, since both $\theta$ and $\alpha$ are smaller.

Additional measurements indicated that the thermoelectric coefficients may be sensitive to cold working and nuay be changed by exposure to high temperature. For Evanohm and Karma significant changes were ob-
TABLE IV

TherMoelectric Data AND ERrors

\begin{tabular}{|c|c|c|c|c|}
\hline \multirow[b]{2}{*}{$\begin{array}{l}\text { Metal } \\
\text { or Alloy }\end{array}$} & \multirow[b]{2}{*}{$\begin{array}{c}\alpha, \mu V /{ }^{\circ} \mathrm{C}^{*} \\
\text { at } 400^{\circ} \mathrm{K}\end{array}$} & \multirow[b]{2}{*}{$\underset{\mu \mathrm{V} /{ }^{\circ} \mathrm{C}}{S}$} & \multicolumn{2}{|c|}{ Calculated Error in a TE } \\
\hline & & & $\begin{array}{c}\mathrm{Dc} \\
\text { reversal** } \\
\Delta I / I, \text { ppm }\end{array}$ & $\begin{array}{c}\text { Ac-dc } \\
\text { difference } \\
\delta_{t}, \text { ppm }\end{array}$ \\
\hline 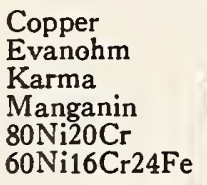 & $\begin{array}{l}-2.0 \\
-1.5 \\
-1.0 \\
-3.0 \ddagger \\
-3.9 \\
-2.3\end{array}$ & $\begin{array}{l}\overline{+0.3 \dagger} \\
+1.8 \\
-1.8 \\
+4.4 \\
+0.8\end{array}$ & $\begin{array}{l}-\overline{200} \\
150 \\
400 \\
500 \\
300\end{array}$ & $\begin{array}{l}-1 \\
<1 \\
-4 \\
-7 \\
-2\end{array}$ \\
\hline
\end{tabular}

* $\alpha$ (Thomson coefficient) is proportional to the absolute temperature.

$\dagger S$ is Seebeck coefficient vs. copper.

This was quite dependent on heat treatment.

From Thomson heating, with $\theta=150^{\circ} \mathrm{C}$ and $\rho k=25 \times$

$10^{-6} \mathrm{~V}^{2} /{ }^{\circ} \mathrm{C}$ at $500^{\circ} \mathrm{K}$, the weighted average temperature of the heater.

** With the bead off center (see text).

served at $800^{\circ} \mathrm{C}$, but not at $400^{\circ} \mathrm{C}$. Thus, care must be taken in controlling the temperature of the wire near welded joints and when out-gassing during the construction of TEs. Here the use of TEs in the reference group by four different manufacturers having differing techniques provided powerful assurance against unknown errors.

As indicated by Wilkins, the thermoelectric errors are greatly reduced in the NPL TEs because of the bifilar heater and the low temperature rise (about $10^{\circ} \mathrm{C}$ at 20 $\mathrm{mA}$ in the present TEs). The resulting error should be much less than $1 \mathrm{ppm}$, even with nickel-chromium heaters.

Rather unfortunately, commercial TEs have lead-in wires (see Fig. 1), through the glass bulb to the heater, of a copper-coated nickel alloy to match the thermal expansion coefficient of the glass. The combination has a rather uncertain but fairly large Seebeck coefficient vs. the heater alloys used. With direct current applied, Peltier heating and cooling of the junctions of these leadin wires and the heater can cause a temperature difference ( + at one junction and - at the other). Most of this heat is conducted by the lead-in to the thick glass press or disk which forms the base of the glass bulb. "This can be considered as a "heat sink" so that the first-order junction temperature change is $\Delta \theta=T I S / K$, where $T$ is the absolute temperature, $I$ is the current, $S$ is the Seebeck coefficient, and $K$ is the thermal conductunce of the lead-in. Widdis showed that this leads to an ac-elc difference term $\delta_{t p}=\alpha \Delta \theta / V_{h}$ where $\alpha$ is the Thomson coefficient and $V_{h}$ is the heater voltage. There 111.11, in addition, be a second-order term at the midpoine $l x$ cause the Peltier heat itself depends on the junction temperature. This can be calculated by carrying ont the second-order approximation mentioned hy Hermuch in Appendix 2 of reference [4] to give, with reasonululs accuracy, $\delta_{p}=+\Delta \theta^{2} / T_{0} \theta$, where $T_{0}$ is the heil-sink lem perature. Of course, $\Delta \theta$ can lee calculated only vers roughly, but this is sufficient. For a typical $10111.1 \mathrm{TH}$ 
it is less than $0.01^{\circ} \mathrm{C}$, and the Peltier errors, $\delta_{t p}$ and $\delta_{p}$ (independent of frequency), are less than $1 \mathrm{ppm}$. This has been verified by comparisons with Evanohm-heater TEs which had copper lead-in wires butt-welded to the nickel alloy wires at the glass press. However, $\delta_{p}$ in particular could be significant in higher-range TEs.

\section{Low- ANd High-Frequency ERrors}

As the frequency is lowered, the temperature of a TE begins to follow the cyclic fluctuations of the square of the heater current. If the TE is not a linear device, the average temperature rise may no longer be the same as at higher frequencies. Hermach's solution of the resulting nonlinear differential equation for the temperature rise has been verified experimentally [4], at least for high-range TEs having large heaters in which the heat abstracted by the thermocouple is negligible. However, for the low range TEs we are concerned with now, these formulas may not apply. The solutions for such cases would be even more difficult, and have not been attempted, particularly since the thermal mass of the bead should then also be considered. In the simpler case, Hermach showed that the ac-dc difference is $\delta_{l}=h \theta d^{2} / \omega^{2} l^{4}$, where $d$ is the diffusivity of the heater alloy, $2 l$ is the heater length, $\omega=2 \pi f$, where $f$ is the frequency, and $h$ is a factor expressing the nonlinearities. The same relationship might be expected for the more complicated case, but with a different numerical factor. Thus, the agreement to better than $10 \mathrm{ppm}$ among the TEs at $20 \mathrm{~Hz}$, shown in Table I, with their varying lengths, gives strong verification to the absence of significant low-frequency errors down to this frequency.

These reference TEs have heaters less than 1 by $10^{-3}$ cm in diameter, with resistivities of $120 \mu \Omega \mathrm{cm}$. The computed errors from skin effect are much less than 1 ppm even at $50 \mathrm{kHz}$. The ac and dc conductances and the capacitance between binding posts on the phenolic top of the box in which the TEs are mounted are much less than the limiting values of $0.01 \mu \mathrm{A} / \mathrm{V}$ and $100 \mathrm{pF}$, respectively, which might cause $1 \mathrm{ppm}$ errors at audio frequencies.

Reactance and skin-effect errors in the resistor units of the TVCs, computed according to formulas developed by the authors [3], are also less than 1 ppn through 50 $\mathrm{kHz}$ up through the 100 volt range, and the comparisons verify this. They cannot easily be calculated for the higher ranges, which have several resistors and adjustable inner shields. Additional reactance errors can arise from the lumped capacitance $C$ of the connector between the resistor and the TE. This shunts the heater, which at these frequencies can be considered as al lumped resistor $R$ in series with an equivalent lumped inductance $L$. It can be shown that the resultant effect of this capacitance on the magnitude of the heater current approaches $\alpha^{2} / 2-\alpha \beta$ as the series resistance (voltage range) is increased, where $\alpha=\omega C R$ and $\beta=\omega L / R$ ( $\alpha$ and $\beta \ll 1)$. The computed error of less than 1 ppun at 5) kllz has heen verified by deliberately doubling the rapacilance.

\section{Other ERrors}

Peltier effects at the heater and lead-in junctions can also cause a second-order change in the effective dc resistance of a TE. The calculation is difficult and the results uncertain, but the change should be much less than one ppm for the 5 to $20 \mathrm{~mA}$ TEs in the NBS reference group. Thus, this cannot account for the somewhat larger changes in effective dc resistance implied by the voltage comparisons of the TEs alone (without series resistors) in the TVCs. Even larger and more puzzling changes have been observed in some other TEs. There is good evidence that they may be caused by rectification at oxide films, which may persist in spite of the welded joints, but there is as yet no direct proof of this.

Dc reversal errors $(\Delta I / I$ for the same EMF) provide a good clue in studying these secondary effects. As shown in Table IV, $\Delta I / I$ calculated by Widdis' formulas should not exceed, perhaps, $200 \mathrm{ppm}$ with Evanohm or Karma, and $500 \mathrm{ppm}$ with nickel-chromium heaters, even if the thermocouple is mounted off the midpoint by 5 percent of the length of the heater (which is easily noticeable). Larger differences are, thus, a sign of other difficulties.

As Hermach showed [4], the current from the heater to ground through the bead of an ungrounded TE can cause heating of the bead and, thus, an error. This is difficult to compute because power dissipated in the bead is somewhat more effective in raising the thermocouple temperature than that in the heater, and the currents in the two halves of the heater are no longer identi$\mathrm{cal}$. However, rough calculations indicate that with one volt across the bead of $\mathrm{TE}_{2}$ in the circuit of Fig. 3 , the bead resistance of a $10 \mathrm{~mA} \mathrm{TE}$ must be greater than 500 megohms if the error is to be less than $1 \mathrm{ppm}$. For the bead materials commonly used, the resistance is a sharp function of temperature and is much less at audio frequencies than with direct current. At NBS, bead resistances are normally measured with a $2 \mathrm{kHz}$ ac bridge with rated direct current through the heater of a TE. The results have been verified (within a factor of 2) by ac-dc comparisons of pairs of TEs with added resistance between the two heaters to significantly increase the bead voltage.

\section{$\mathrm{X}$. Conclusions}

A reference group of two very different kinds of TEs has been established. The authors believe that it is very unlikely that the average ac-dc difference of the group differs from zero by more than $2 \mathrm{ppm}$ at audio frequencies. This is based on the agreement to better than $0.5 \mathrm{ppm}$ of the averages of the two kinds of TEs, and on allow: ance of $1 \mathrm{ppm}$ for the uncertainty of the average in the network of comparisons. The results are in excellent agreement with calculations, which show that the ac-dc differences should not exceed 1 ppm. With the precise techniques that have been developed, pairs of TEs of the group maly be compared with a $3 \sigma$ uncertainty of about 2 ppm without any evidence to date (in over three yeirs of measurements) of significant 
systematic errors. This is somewhat remarkable, since Ructuations in the best available wide-range ac and dc sources greatly exceed this, and most of these TEs have rather large residual thermal EMFs and have temperature coefficients of EMF exceeding $1000 \mathrm{ppm} /{ }^{\circ} \mathrm{C}$.

Adjacent ranges of the TVCs may also be compared with the same precision to step up and down the voltage scale. Their ac-dc differences can be determined to better than $10 \mathrm{ppm}$ when used with TEs of known ac-dc differences, again without evidence of systematic error. Alernating voltage measurements can be made to 20 ppin or better by using the TVCs or ac-dc transfer standards with appropriate dc standards.

This accuracy and precision in the ac-dc transfer, more than ten times better than heretofore available, are good indications of the virtue of simplicity in reference standards. Properly designed and constructed TEs are rather simple devices that correspond to the desired idealized mathematical models remarkably closely, thus providing the agreement between theory and experinient which is so desirable in a development such as this.

The agreement to better than 1 ppm between the conventional $\mathrm{TEs}$ and the new very different multijunction NPL TEs (which should theoretically have smaller errors), in addition to providing semi-independent verification of the accuracy of both, indicates that even higher accuracy may be possible in the future with these new designs. Conventional single-junction TEs could be improved by the use of bifilar heaters and closely spaced lead-in wires with good thermal conductance between the two halves of the heater and between the two lead-ins. In this way, other heater alloys could be used.

Well-designed and well-constructed ac-dc transfer standards can be remarkably stable over most of their frequency range. Most factors which may change the response, such as temperature and dimensional changes, have little or no effect on the ac-dc difference. There is already some evidence that long-time stabilities of a few ppm can be attained in properly designed and constructed TEs and TVCs. However, we have noted an apparently abrupt change of $+5 \mathrm{ppm}$ in one TE, accompanied by a change of over $100 \mathrm{ppm}$ in its dc reversal difference. Thus, TEs should be compared frequently if the highest accuracy is desired.

Comparisons of adjacent ranges, with a step-up procedure such as the one described, can be used to guard against unexpected changes in TVCs. This has already been verified in the design and construction of two somewhat similar sets of TVCs by W. Scott, K. Ballard, and D. Bailey of the NBS Electronic Calibration Center, Boulder, Colo. These units incorporate large wirewound resistors of controllable reactances and low temperature coefficients, so that they do not require troublesome forced-air cooling. They will be described elsewhere.

The step-up procedure is also feasible with simpler sets of TVCs incorporating fewer plug-in resistor units and two TEs of different current ranges. Two such sets have been built but have yet to be completely evaluated.
APPENDIX I

\section{EMF COMPARATOR}

If the thermocouple resistance $R_{t 1}$ of $\mathrm{TE}_{1}$ (Fig. 3) is much less than $R_{1}+R_{2}$, the EMF $E_{1}$ is substantially the same with alternating and direct currents applied during the test (the procedure involves holding the voltage at the output terminals of $\mathrm{TE}_{1}$ constant). Then, from the definition of ac-dc difference, $\left(I_{a}-I_{d}\right) / I_{d}=\delta_{1}$. Similarly, for $\mathrm{TE}_{2}\left(I_{a}-I_{d}{ }^{\prime}\right) / I_{d}{ }^{\prime}=\delta_{2}$, where $I_{d}{ }^{\prime}$ is the direct current that would be required to produce the same EMF of $\mathrm{TE}_{2}$ as $I_{a}$. The change $D_{a}-D_{d}$ in detector $D_{1}$ which occurs when $I$ is changed from $I_{a}$ to $I_{d}$ ( $I_{d}$ being adjusted to keep $E_{1}$ constant) results from the change in $E_{2} . D_{a}-D_{d}$ may then be interpreted as a measure of the departure of $I_{d}$ from the value $I_{d}{ }^{\prime}$ which would have produced the same $E_{2}$ as would $I_{a}$. Thus, combining the above two equations and neglecting second-order terms, we have $\left(I_{d}{ }^{\prime}-I_{d}\right) / I_{d}=\delta_{1}-\delta_{2}$.

The sensitivity of the detector to small current differences in $\mathrm{TE}_{2}$ can be determined by shunting its heater $R_{h}$ with a resistor $R_{\varepsilon} \gg R_{h}$ to cause a small change in its heater current $\Delta I$, noting the change in deflection $\Delta D$ of $D_{1}$ and computing $s=\Delta I / I \Delta D\left(\Delta I / I=R_{h} / R_{s}\right.$ if the heater current of $T E_{1}$ is unchanged). Then, if $s$ is constant over the range of deflections involved, we have $\delta_{1}-\delta_{2}=s\left(D_{a}-D_{d}\right)$, as in the text. Similarly, in the comparison of two voltage converters, a resistor $R_{s} \ll R_{m}$ can be added in series with $\mathrm{TVC}_{2}$ to give proportional change in applied vol tage $\Delta V / V=R_{\varepsilon} / R_{m}$, where $R_{m}$ is the TVC resistance. Then $s=\Delta V / V \Delta D$.

These two calibration methods must leave the current in $\mathrm{TE}_{1}$ unchanged. The change $\Delta I$ can of ten be introduced and measured more conveniently in some way (as by changing the supply voltage) that changes the current in both TEs by a small known amount. We have found it convenient to disconnect $E_{1}$, set the voltage of $P$ to the same value, short $D_{2}$, observe the resulting $\Delta D$ when $I$ is changed, and compute $s$ as before.

If only a few TEs are ordinarily used in the "standard" position ( $\mathrm{TE}_{2}$ in Fig. 3 ), it is convenient to make use of the approximate output-input relation (at is fixed frequency) for a TE, $\Delta E / E=n \Delta I / I$, where $n$ is dependent on $I$ but not $\Delta I$ if $\Delta I \ll I$, and is close to 2. For ealch such TE, $n$ can be determined in advance at several values of $E$ and plotted vs. $E$, since it is quite stable. Then the sensitivity of the detector in the circuil call bo determined by inserting a small known voluge $\Delta:$ : $\ll 1$ : (with a Lindeck potentiometer, not shown in lig. 3) in series with and opposing $E_{2}$, observing (he change $\Delta /$ ) in $D_{1}$ and computing the factor $k=\Delta D / \Delta E_{\mathrm{p}}$. Then, $\left.\right|_{\text {rom }}$ this and the previous equations $\delta_{1}-\delta_{2}=\left(I_{d}^{\prime}-I_{d}\right) I_{d}$ $=\left(E_{d}^{\prime}-E_{d}\right) / n_{2} E_{2}=\left(D_{a}-D_{d}\right) / k n_{2} E_{2}$. $E_{2}$ is rasily me, sured with potentiometer $P$.

If $R_{t 1}$ is not negligible, $E_{1}$ is not constant during the test, since the currents in $R_{1}$ and $R_{2}$ depend on botle $F_{1}$ and $E_{2}$. The corresponding equations for detcrminmin $\delta_{1}-\delta_{2}$ by means of deflections of $D_{1}$ then contain arld tional correction terms involving the resistances of the several parts of the EMF comparator, and are 100 com 
plex to be useful for routine measurements. They show, however, that if $R_{t 1} \leq\left(R_{1}+R_{2}\right) / 100$, the simplified equations already derived are valid to a few percent of the computed $\delta_{1}-\delta_{2}$. They show that $R_{t 2}$ is not critical. For our comparator, $R_{1}+R_{2}=1100 \Omega$ and $R_{t 1} \leq 10 \Omega$ for the usual TEs.

For each NPL converter, $R_{t} \approx 1000 \Omega$. It is always placed in the $T E_{2}$ position, except when the two NPL converters are intercompared, requiring the use of the more complicated formula.

\section{APPENDIX II}

\section{REs in Parallel}

By using the definitions for $n$ and $\delta$ of a TE (see Appendix I) we have, closely enough for small changes in $E$,

$$
\frac{E_{a}-E_{d}}{n E_{d}}=\frac{I_{a}-I_{d}}{I_{d}}-\delta .
$$

In the ac-dc test, nominally equal resistors are connected in series with $\mathrm{TE}_{a}$ and $\mathrm{TE}_{b}$, and the two branches are connected in parallel. The sum of the two EMFs, $E_{a}+E_{b}$, is held constant. Then, with second subscripts to identity the two TEs, we have

$$
E_{a a}+E_{a b}-E_{d a}-E_{d b}=0
$$

and

$$
\begin{aligned}
& n_{a} E_{d a}\left(\frac{I_{a a}-I_{d a}}{I_{d a}}\right)+n_{b} E_{d b}\left(\frac{I_{a b}-I_{d b}}{I_{d b}}\right) \\
&=n_{a} E_{a} \delta_{a}+n_{b} E_{b} \delta_{b}
\end{aligned}
$$

If the reactances of the two paths are equal

$$
\frac{I_{a a}-I_{d a}}{I_{d a}}=\frac{I_{a b}-I_{d b}}{I_{d b}}=\frac{I_{a}-I_{d}}{I_{d}}=\delta_{p}
$$

where $I_{a}$ and $I_{d}$ are the sums of the alternating and direct currents in the two branches.

If $E_{a}=m E$ and $E_{b}=(1-m) E$,

$$
\left[n_{a} m+n_{b}(1-m)\right]\left[\frac{I_{a}-I_{d}}{I_{d}}\right]=n_{a} m \delta_{a}+n_{b}(1-m) \delta_{b} .
$$

If the reactances of the two paths are not equal, additional terms will be introduced which are difficult to calculate. However, they are zero if $n_{a} m=n_{b}(1-m)$, i.e., if the TEs are matched in response. Then $\delta_{p}$ $=\left(\delta_{a}+\delta_{b}\right) / 2$. Even if the reactances are unequal, the added terms are essentially eliminated if a second test is made with the two resistors interchanged and the results averaged.

\section{ACKNowledgment}

The authors express their appreciation and thanks to F. J. Wilkins for the two multijunction TEs, to L. Julie of the Julie Research Laboratories for suggesting the basic idea of the EMF comparator, and to the following for their cooperation in the manufacture of other TEs used in this group: the Levy brothers of the American Thermoelectric Company, L. R. Graham of Graham Associates, F. Gay of the Best Products Company, M. Rosenfield of the former Field Electrical Instrument Company, and L. W. Pignolet of Weston Instruments Incorporated.

The use of these particular TEs with heaters of Evanohm and Karma should not in any way be construed as constituting an exclusive endorsement by NBS of these products or alloys. Other TEs, made to the same specifications with the same or other alloys of equally low thermoelectric effects, should be equally suitable.

\section{REFERENCES}

[1] "American standard requirements for electrical indicating instruments." New York: American Standards Association, C.39.1, 1964.

[2] F. J. Wilkins et al., "Multijunction thermal converter," Proc. $I E E$ (London), vol. 112, pp. 794-806, April 1965.

[3] F. L. Hermach and E. S. Williams, "Thermal voltage converters for accura te voltage measurements to 30 megacycles per second," Trans. AIEE (Communication and Electronics), vol, 79, pt. I, pp. 200-206, July 1960.

[4] F. L. Hermach, "Thermal converters as ac-dc transfer standards for current and voltage measurements at audio frequencies," J. Res. NBS, vol. 48, pp. 121-138, February 1952.

[5] W. J. Youden, "Measurement agreement comparisons," Proc. 1962 Standards Laboratories Conf. Washington, D. C.: NBS, Misc. Pub. 248, August 16, 1963, pp. 147-152.

[6] F. C. Widdis, "The theory of Peltier and Thomson effects in thermal ac-dc transfer devices," Proc. IEE (London), Monograph 497M, January 1962.

[7] Landolt-Bornstein, "Zahlenwerte und Funklionen aus Physik, Chemie, Astronomie, Geophysik und Technik," 6. Auf., Band II/6, (6th ed. vol. 2, pt. 6). Berlin: Springer-Verlag, 1959.

[8] D. Flach and L. A. Marzetta, "Calibration of peak a-c to d-c comparators," Proc. 1965 ISA Conf., pt. 1, vol. 20, paper 14. 


\title{
Thermal Voltage Converters and Comparator for Very Accurate AC Voltage Measurements
}

\author{
E. S. Williams \\ Institute for Basic Standards, National Bureau of Standards, Washington, D.C. 20234
}

(July 22, 1971)

\begin{abstract}
A new fourteen-range set of thermal voltage converters and a thermoelement comparator are used to measure ac-dc difference, and a-c voltages relative to external d-c standards, with $20 \mathrm{ppm}$ (parts. per-million) accuracy at audio frequencies. The imprecision is less than $2 \mathrm{ppm}$. Corrections relative to the very stable middle ranges can be redetermined for every range by a seven-step intercomparison of certain adjacent ranges.
\end{abstract}

Key words: AC-DC difference; comparator; thermoelement; transfer voltmeter; voltage measurements.

\section{Introduction}

An a-c voltage at audio frequencies is measured most accurately at the present time by using a thermal voltage converter (TVC) to compare it with a stable and accurately measured $\mathrm{d}$-c voltage, which is nominally equal to it. The basic d-c standards are then, in effect, "transferred" to the a-c measurement. The TVC may be simply a thermoelement (TE) in series with an appropriate multiplier resistor. The outpui emf of the TE is ordinarily monitored with a null detector and a balancing circuit, which may be a Lindeck potentiometer. A balancing circuit and a null detector are included in most commercial multirange models.

Before the TVC is used for a-c voltage measurements it must be tested for ac-dc difference or frequency in. fluence so that corrections may be applied. The set of TVCs and the new TE comparator described in this paper were developed primarily for making these tests. However they may also be used for a-c voltage measurements as explained in section 9. For ac-dc difference measurements the TE comparator, whose read out instrument may be either a narovoltmeter or a galvanometer, provides a considerable advantage over other methods in overcoming the difficulties caused by power supply instability and inexact voltage control.

The 14-range set of TVCs (designated No. 7) consists of six resistor units and two TEs, and extends from 1 to $1000 \mathrm{~V}$. The TEs are rated at 2.5 and $5.0 \mathrm{~mA}$, and each one may be attached to any one of the resistors by a coaxial connector (see fig. 1). This permits each resistor to be used for two voltage ranges, as shown in table 1. Certain adjacent ranges of the set can be intercompared, and the ac-dc differences of all the ranges can be determined relative to any one range. This set is otherwise similar to an earlier set $[1]^{1}$, which consisted of two $5-\mathrm{mA}$ TEs and 12 series resistors, and covered a range from 0.5 to $500 \mathrm{~V}$. The earlier set, designated No. l, has been extended to $1000 \mathrm{~V}$ with one additional resistor as part of the present project. It is evaluated in a 14-step intercomparison in which each range is compared, at reduced voltage, with the next lower one.

In a well designed TVC the ac-dc difference is not affected by changes in the applied voltage. Therefore, an ac-dc difference determined for the $100-\mathrm{V}$ range at $60 \mathrm{~V}$ can be applied as a correction when this resistor is used at $200 \mathrm{~V}$ to test the nominal $300 . \mathrm{V}$ range of the next higher resistor. Experience has shown this stepup procedure to be feasible. However if the higher voltage ranges are not carefully built the ac-dc differences can change due to self-heating effects to be described later.

\section{Design and Construetion}

The two TEs are mounted in 2 -in brass tubes (see figs. 1 and 2 , upper right) with coaxial connectors for attaching to a resistor. The $2.5 \mathrm{~mA}$.TE has a $400 \Omega$ heater and is used alone as the $1 . V$ range. The $5 \mathrm{~mA}$.TE has a $125 \Omega$ heater and a $275 \Omega$ resistor is added inside its enclosure to make a $400 \Omega, 2-\mathrm{V}$ range.

The middle ranges (six ranges, 3 to $60 \mathrm{~V}$ ) make use of three resistors $-0.8 \mathrm{k} \Omega, 3.6 \mathrm{~kJ}$ ), and $12 \mathrm{k} \Omega$ (see table 1). Each of these is a $2 W$ metal.film resistur mounted coaxially in a 3 -in brass cylinder $41 / 2$ in long with coaxial connectors at each end.

\footnotetext{
' Figures in brackets indicate the literature referenees on pase 153
} 


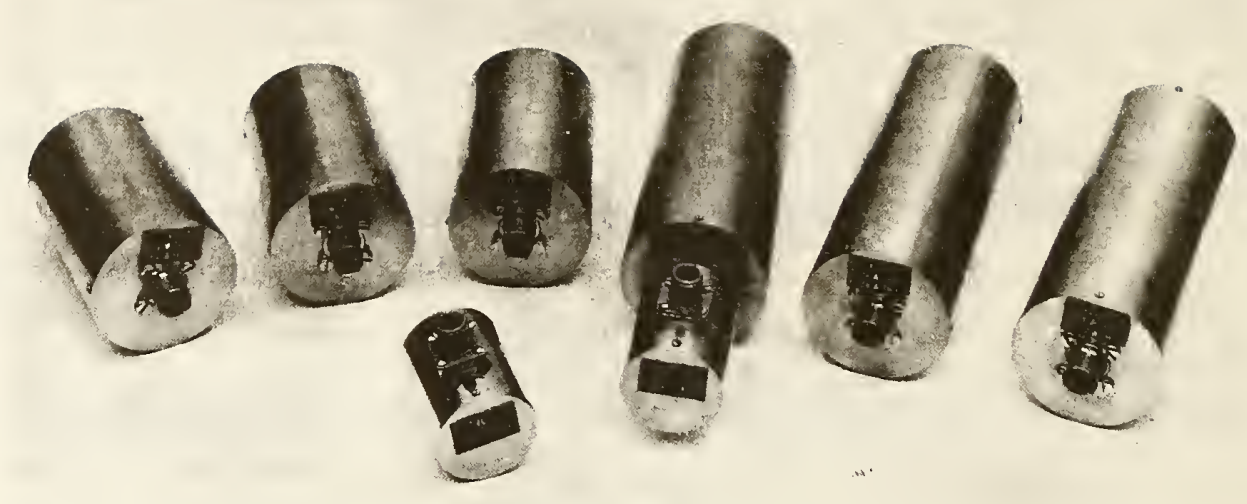

Figure 1. New set of thermal voltage converters.

The simple symmetrical geometry of these units permits approximate calculations of their reactances [2]. The calculations are inexact because of the necessary assumptions as to end-effects, and they neglect any small residual reactances of the resistors themselves. They do indicate however, that the frequency error of each resistor unit (without the TE) should be less than $1 \mathrm{ppm}$ at $50 \mathrm{kHz}$, even for tubes smaller than 2 in in diameter. (The 3 -in tube was chosen for the higher ranges where more space is necessary and was therefore used for the middle ranges also.)

The higher voltage ranges have larger resistor assemblies to avoid excessive temperature rise. The $100-200 \mathrm{~V}$ unit has a $40 \mathrm{k} \Omega$ spiraled metal-film resistor five inches long. ${ }^{2}$ The $300-600$ and $500-1000 \mathrm{~V}$ units have resistors of tin oxide deposited on a 0.81 -in glass tube 6 in long. A small voltage coefficient was anticipated in these resistors, but measurements have shown it to be negligible.

"This experimental resistor is not ordinarily available commercially. Four $40 \mathrm{k} \Omega$ metal film resistore in series.parallel can also be satisfactory.

TABLE 1. NBS thermal voltage converter set No. 7

Fourteen voltage ranges (column 2) are formed with six series resistors and two TEs (F 1 and $F 2$ ).

\begin{tabular}{|c|c|c|c|}
\hline $\begin{array}{l}\text { Series } \\
\text { resistor }\end{array}$ & $\begin{array}{c}\text { Voltage } \\
\text { range }\end{array}$ & $T E$ & $\begin{array}{c}\text { Total } \\
\text { resistance }\end{array}$ \\
\hline$k \Omega$ & & $m A$ & $k \Omega$ \\
\hline & 1 & $2.5(\mathrm{Fl})$ & 0.4 \\
\hline & 2 & $\begin{array}{l}3.0(1 \times 2) \\
25\end{array}$ & 0.4 \\
\hline $0.8 \ldots$ & 6 & 5.0 & 1.2 \\
\hline & 10 & 2.5 & 4.0 \\
\hline $3.6 \ldots \ldots$. & 20 & 5.0 & 4.0 \\
\hline & 30 & 2.5 & 12.4 \\
\hline $12 \ldots \ldots \ldots$ & 60 & 5.0 & 12.4 \\
\hline 40 & $\begin{array}{l}100 \\
200\end{array}$ & $\begin{array}{l}2.5 \\
5.0\end{array}$ & $\begin{array}{l}40.4 \\
40.4\end{array}$ \\
\hline & 300 & 2.5 & 120.4 \\
\hline $120 \ldots$ & 600 & 5.0 & 120.4 \\
\hline & 500 & 2.5 & 200.4 \\
\hline $200 \ldots$ & 1000 & 5.0 & 200.4 \\
\hline
\end{tabular}

Errors in these ranges are caused mainly by capacitance between the resistor assembly and the outer casing, which permits alternating current to bypass the TE to ground. Therefore more a-c than d-c voltage is required for a given TE output. Frequency compensation could be provided by placing relatively small capacitors in parallel with part of the resistance. However, such capacitors probably would not be sufficiently stable over a long period of time and might be affected by temperature changes which occur in the resistor enclosure.

Compensation was therefore provided (as in the earlier set No. 1) with an inner shield which is connected to the input and surrounds the high- or input. end of the resistor (see "S" in fig. 2). The shield is positioned, relative to the resistor, to control the capacitance currents and provide optimum high-frequency
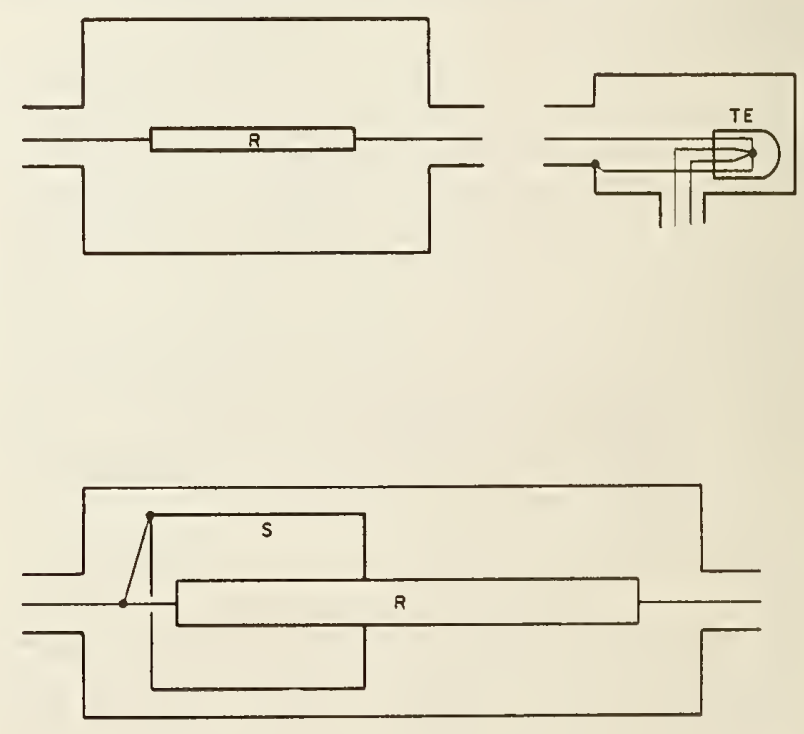

FIGURE 2. Essentials of thermal voltage converters. Thermoelement (TE) enclosure, low voltage unit with resistor $(R)$, and high voltage unit $(100$ to $1000 \mathrm{~V})$ with resistor $(\mathrm{R})$ and frequency compensation shield (S). 
compensation; perfect compensation is possible only at one frequency.

\section{1000-V TVC}

As stated earlier the corrections for the high and low voltage ranges are determined relative to the middle range by intercomparison tests. It is necessary therefore that the ac-dc difference of all ranges be unaffected by voltage level. The voltage effect is rare in low and middle ranges, but it can be troublesome at higher ranges where the heat generated by the resistor is appreciable. All intercomparison tests were initially inade at two voltage levels in order to evaluate this effect-or to prove its absence. If, for example, the nominal $600 . \mathrm{V}$ range is compared with the $1000-\mathrm{V}$ range at both 400 and $600 \mathrm{~V}$, and the same results are obtained, it is unlikely that the $600-\mathrm{V}$ range is affected by the voltage increase (the $1000-\mathrm{V}$ range is very probably unaffected at these voltages). However both ranges are in question until we are assured that the $1000-\mathrm{V}$ range is also free of this effect.

The highest range, then presents a special problem. It is more likely to change due to increased voltage (heating) and there is no higher range to compare it with. If it is compared with another 1000 .V TVC at two or more voltages and no change is observed it is possible that both have the same voltage effect. However this is unlikely if TVCs are of somewhat different design. If intercomparisons of several units of difference design show no change between rated voltage and a lower voltage we may safely assume this effect is negligible in each one.

Five 1000-V TVCs (table 2) have therefore been built, each with differing components, and a network of intercomparison tests was made as described in the next section. The original set of TVCs [1] was included in these tests.

TABLE 2. Five 1000.V TVCs

\begin{tabular}{|c|c|c|c|c|}
\hline \multirow[t]{2}{*}{ Unit } & \multirow[t]{2}{*}{ Resistance } & \multicolumn{2}{|c|}{ Voltage range with $T E$} & \multirow[t]{2}{*}{ Resistorassembly } \\
\hline & & $F 1$ & $F 2$ & \\
\hline A & $\begin{array}{l}k \Omega \\
400\end{array}$ & 1000 & & $\begin{array}{l}\text { Four } 100 \mathrm{k} \Omega \text { wire- } \\
\text { wound resistors in } \\
\text { series, each } 13 / 8 \text { in } \\
\text { long. }\end{array}$ \\
\hline B & 400 & 1000 & & $\begin{array}{l}\text { Four } 100 \mathrm{k} \Omega \text { wire- } \\
\text { wound resistors in } \\
\text { series, each } 13 / 8 \text { in } \\
\text { long. }\end{array}$ \\
\hline C & 200 & 500 & 1000 & $\begin{array}{l}\text { Four } 200 \mathrm{k} \Omega \text { metal- } \\
\text { film resistors in } \\
\text { series-parallel, each } \\
2 \text { in long. }\end{array}$ \\
\hline D & 200 & 500 & 1000 & $\begin{array}{l}\text { One } 200 \mathrm{k} \Omega \text { tin oxide } \\
\text { resistor } 6 \text { in long. }\end{array}$ \\
\hline $\mathbf{E}$ & 400 & 1000 & & $\begin{array}{l}\text { Four } 400 \mathrm{k} \Omega \text { metal- } \\
\text { film resistors in } \\
\text { series-parallel, each } \\
2 \text { in long. }\end{array}$ \\
\hline
\end{tabular}

The five $1000 \cdot \mathrm{V}$ TVCs are designated alphabetically (A through $E)^{3}$ and all except " $A$ " have the inner shield, as described below, for frequency compensation. The resistor in unit " $\mathrm{A}$ " is mounted concentrically in a set of six brass rings whose potentials are maintained by a capacitance divider. The whole assembly is mounted in a 4-in brass cylinder 7 in long.

Self-heating of a TVC may affect either the frequency compensation or the resistor itself. Apparently the dielectric losses in the insulation between conductors can be affected by heating and change the impedance of the resistor. This change occurred within a few minutes after a voltage increase or decrease in several resistor types, which were therefore discarded.

The frequency compensating shield may be moved, relative to the resistor, by thermal expansion of mounting parts and cause a change in ac-dc difference. This occurred in some of our early units and the change was slow, taking up to an hour. Later shields were therefore mounted very rigidly since a small displacement will have a large effect on the frequency influence.

The shield now in use is in two parts. A brass cylinder, with one end closed, is mounted firmly against the outer end piece (input end) with a polystyrene insulator one-half inch thick (fig. 3). A movable tube fits tightly inside this cylinder and the shield length adjustment is made by pushing this piece forward with a small rod inserted through the end piece and the insulator. The rod is removed after each adjustment.

The shield is most effective at the leading edge where the voltage difference is greatest between the resistor and the shield. Therefore the movable part of the shield is cut at an angle, so that only part of it (see fig. 3) extends outside the fixed cylinder. Thereby the effect of small position changes is reduced. Even so, the compensation is affected by approximately 0.01 percent per millimeter of position change in some typical units.

The shield length adjustment is made in small steps, and tests are made after each adjustment. When optimum compensation is achieved, the unit is opened and the shield parts are locked together with three screws through their overlapping portions.

\section{TE and TVC Tests}

As stated earlier the middle ranges are the most accurate, and they are the base from which higher and lower ranges are tested. However they are no more accurate than the TE used with them. Before being installed in a TVC the TEs are therefore compared with one of a group of carefully selected TE standards which were made according to NBS specifications. This group contains twelve elements made by four manufacturers, and are rated from 5 to $20 \mathrm{~mA}$. [1] They have either Karma or Evanohm ${ }^{4}$ heaters in reduce

\footnotetext{
"Unit "D" is assigned to this set (No. 7).

- Certain commercial products and inatrumente ore idrntified in the parwo wa ordet 10

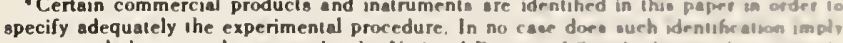
recommendation or endorsement by the National Burrau of Siandarde, nor dese 11 inply that the products or equipment identified are necensarly the beat avalable for the purpone
} 


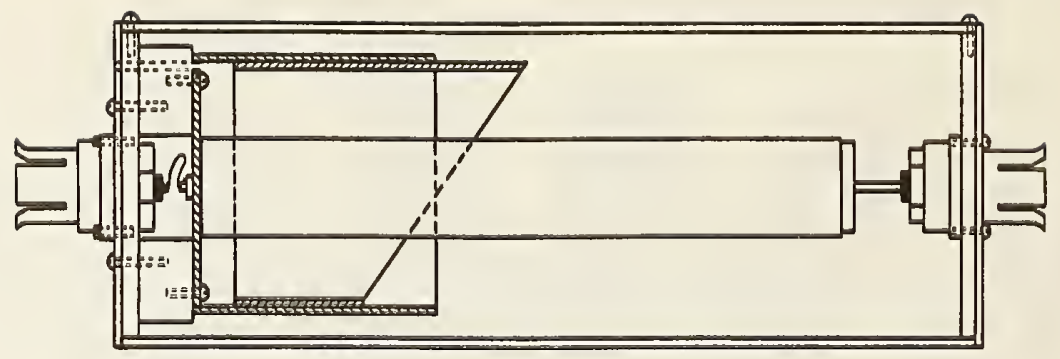

Figure 3. Cross section of high voltage TVC.

Input end to at the left. Fixed and adjuotable shleld parts sre eupported by a polystyrene lneulator one.half inch thick.

thermoelectric effects in the heaters. They also have small reverse d-c differences (less than $200 \mathrm{ppm}$ ), high bead resistances (over $1000 \mathrm{M} \Omega$ ), and small ac-dc differences. An additional two are multi-junction elements of different design. All of these TEs have been intercompared as current converters with agreement to better than $3 \mathrm{ppm}$. Theoretical calculations, taking into consideration all known sources of error, also indicate that the error should not exceed a few $\mathrm{ppm}$ at audio frequencies. Because of this and because of the excellent agreement between these thermo. elements of different design and construction, we may safely assume that the average ac-dc difference of the group is 0 to $\pm 2 \mathrm{ppm}$.

The low-frequency performance of a TVC depends on the TE since the reactance of the resistor is entirely negligible even at a few kilohertz. The accuracy of a TE at low frequency is mainly dependent on the length of the heater. A very short heater permits heat to flow more readily to the support stems and cooling to occur between current peaks. However most com. mercial TEs have errors less than $10 \mathrm{ppm}$ at $20 \mathrm{~Hz}$ and some have errors less than $10 \mathrm{ppm}$ even at $5 \mathrm{~Hz}$.

In testing selected commercial TEs for the TVCs at audio frequencies we often find a small ac-dc difference (up to $4 \mathrm{ppm}$ ) which is independent of frequency but dependent on heater current. It ordinarily decreases at lower heater currents. When these TEs are used in a TVC the ac-dc difference will therefore be slightly voltage dependent. In the step-up test process, where the TVC is alternately used at 50 and 100 percent of rated current, this relatively small thermoelement error is introduced at every step of the process. If the process is a long one, as in the 12-resistor set, there could be a significant accumulation of error. TVCs in the new set are intercompared by testing each resistor once with one TE at reduced current ( 60 or $67 \%$ ), and again with rated current on the other TE. If these TEs differ in ac-dc difference at these currents, there will again be an accumulation of error.

This is one of the advantages of the set with only six resistors. If the $20 . \mathrm{V}$ range is the starting point (it is probably the most stable), we have only four steps up to the $1000 . \mathrm{V}$ range, and three steps down to the $1-V$ range. The other advantages are that less time is required for the intercomparisons and there are fewer units to construct.
Intercomparison data for this set of TVCs are listed in table 3 as well as determinations made using TVC set No. 1. (Test methods are discussed in later sections of the paper). The middle ranges differ by $2 \mathrm{ppm}$ or less even though the resistance varies from 1200 to $12000 \Omega$. Since the resistance enters into the theoretical calculation of ac-dc difference, this agreement is an additional indication of accuracy.

Step-down tests show that the two lowest voltage ranges usually have a positive correction (i.e., more a-c than d-c voltage is required for a given $\mathrm{TE}$ output) of $5 \mathrm{ppm}$ or less at $50 \mathrm{kHz}$. There is no resistor in series with the TE at the $1 \mathrm{~V}$ range, and at the $2 . \mathrm{V}$ range the TE is a large part of the total impedance. These ranges are therefore affected more by the small reactance of the TE. The corrections for these ranges are more likely to change if a TE is replaced than for the higher ranges. A step-down test from the middle range is therefore advisable when TE replacements are made.

The five $1000 . \mathrm{V}$ TVCs were compared with the 300 . and $500 . \mathrm{V}$ ranges of TVC set No. 1 and the $600 . \mathrm{V}$ range of the new set No. 7. The corrections are listed in table 4 for 20 and $50 \mathrm{kHz}$ and all values are averaged.

TABLE 3. $A C \cdot D C$ difference

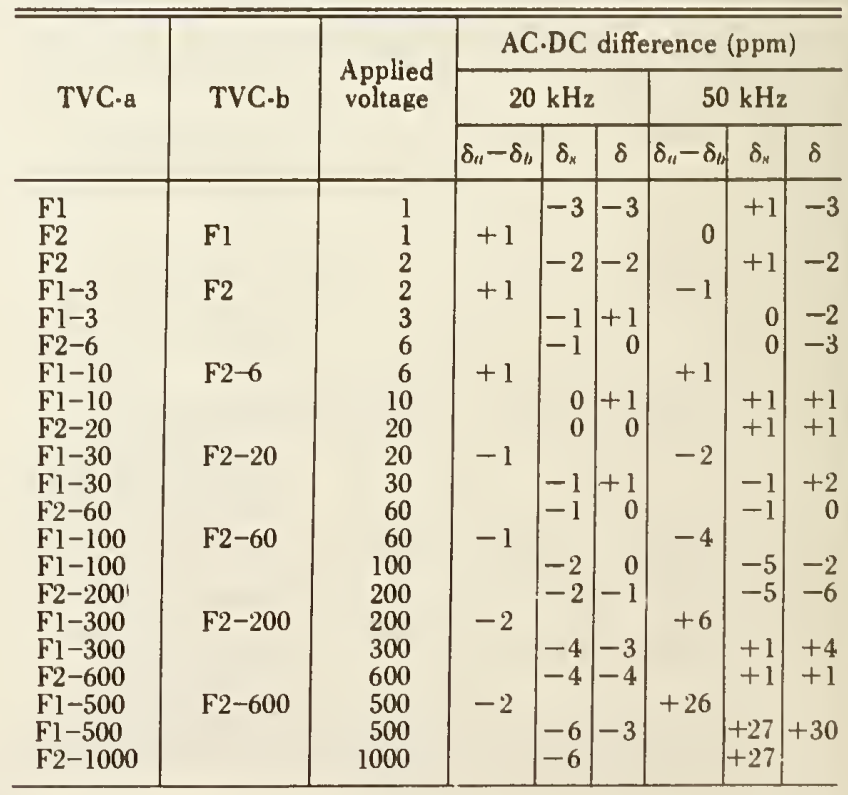


Table 5 lists intercomparison data for the same frequencies for seven pairings of the five 1000-V TVCs. Values for 600 and $1000 \mathrm{~V}$ do not differ significantly and so they are all averaged together.

Corrections $(\delta)$ for each range of the new TVC set are listed as determined in comparison with TVC Set No. 1. Intercomparison data $\left(\delta_{a}-\delta_{b}\right)$ are also listed and a computed correction $\left(\delta_{s}\right)$ based on the intercomparison. starting with the average of the measured ac-dc differences of the 10 - and $20-\mathrm{V}$ ranges. The TVCs are identified as the combination of a TE (F1 or F2) with the appropriate resistor; i.e., F2-600 forms the $600 . \mathrm{V}$ range.

Averages of the corrections for the five $1000-\mathrm{V}$ TVCs listed in table 4 are shown in the circles in figure 4 , and the connecting lines show the intercomparison values from table 5 . The agreement between the various units is illustrated by this figure.

Each of the values listed in tables 4 and 5 is the average of at least four separate determinations. An analysis of the data taken in a series of 26 intercomparison tests (an average of 5 determinations witl each one), involving all ranges at two frequencies and usually two voltages, showed an imprecision ( $3 \sigma_{a}$, where $\sigma_{a}$ is the standard deviation of the average of five determinations) of only $1.4 \mathrm{ppm}$.

TABI.E 4. Ac-dc difference corrections for five 1000-volt TVC (A-E) The standard instruments are the 300 - and $500-\mathrm{V}$ units of Set No. 1 and the $600 . \mathrm{V}$ range of Set No. 7.

\begin{tabular}{|c|c|c|c|c|c|c|}
\hline Standard & Volts & A & B & C & D & $\mathrm{E}$ \\
\hline \multicolumn{7}{|c|}{ AC.DC difference at $20 \mathrm{kHz}(\mathrm{ppm})$} \\
\hline $\begin{array}{l}5 m-1 \\
300-1 \\
600-7 \\
\text { Ave. }\end{array}$ & $\begin{array}{l}500 \\
300 \\
600\end{array}$ & $\begin{array}{l}-3 \\
-8 \\
-6\end{array}$ & $\begin{array}{l}-20 \\
-22 \\
-21\end{array}$ & -9 & $\begin{array}{l}-4 \\
-4 \\
-2 \\
-3 \\
-3\end{array}$ & $\begin{array}{c}-12 \\
-10 \\
-12,-10 \\
-10 \\
-11\end{array}$ \\
\hline
\end{tabular}

AC.DC difference at $50 \mathrm{kHz}(\mathrm{ppm})$

\begin{tabular}{l|l|l|l|l|l|l}
\hline $500-1$ & 500 & +21 & -6 & +11 & +30 & -3 \\
$300-1$ & 300 & & & & -30 & -4 \\
$600-7$ & 600 & +30 & -9 & & +30 & -6 \\
& & +23 & & & +32 & -6 \\
Ave. & & +25 & -7 & +11 & +30 & -5 \\
\hline
\end{tabular}

TABLE 5. Relative ac-dc differences for seven pairings of members of the five 1000-V TVCs

\begin{tabular}{|c|c|c|c|c|c|c|c|}
\hline Volts & $\delta_{\mathrm{A}}-\delta_{\mathrm{B}}$ & $\delta_{\mathrm{B}}-\delta_{\mathrm{C}}$ & $\delta_{\mathrm{C}}-\delta_{\mathrm{D}}$ & $\delta_{\mathrm{D}}-\delta_{\mathrm{E}}$ & $\delta_{\mathrm{E}}-\delta_{\mathrm{A}}$ & $\delta_{\mathrm{A}}-\delta_{\mathrm{C}}$ & $\delta_{\mathrm{A}}-\delta_{\mathrm{D}}$ \\
\hline \multicolumn{8}{|c|}{ AC-DC difference at $20 \mathrm{kHz}(\mathrm{ppm})$} \\
\hline $\begin{array}{l}600 \\
1000 \\
600 \\
1000 \\
\text { Ave. }\end{array}$ & $\begin{array}{l}-11 \\
-17 \\
-16 \\
-17 \\
-15\end{array}$ & $\begin{array}{l}+14 \\
+13 \\
+17 \\
+15\end{array}$ & $\begin{array}{r}+2 \\
0\end{array}$ & $\begin{array}{r}-7 \\
-6 \\
-10 \\
-7\end{array}$ & $\begin{array}{r}+7 \\
0 \\
+4\end{array}$ & $\begin{array}{l}+3 \\
+9\end{array}$ & $\begin{array}{r}0 \\
+5 \\
-2 \\
+5 \\
+2\end{array}$ \\
\hline \multicolumn{8}{|c|}{ AC-DC difference at $50 \mathrm{kHz}(\mathrm{ppm})$} \\
\hline $\begin{array}{l}600 \\
1000 \\
600 \\
1000 \\
\text { Ave. }\end{array}$ & $\begin{array}{l}-20 \\
-22 \\
-25 \\
-24 \\
-23\end{array}$ & $\begin{array}{r}+14 \\
+6\end{array}$ & $\begin{array}{r}+10 \\
+2\end{array}$ & $\begin{array}{l}-33 \\
-33 \\
-37 \\
-34\end{array}$ & $\begin{array}{l}+28 \\
+35\end{array}$ & $\begin{array}{l}-15 \\
-14 \\
-15 \\
-15\end{array}$ & $\begin{array}{l}+3 \\
+4 \\
+2 \\
+1 \\
+2\end{array}$ \\
\hline
\end{tabular}

\section{Thermal Compensation}

The coaxial connector between the TE and the series resistor provides a low-reactance connection for minimum frequency error. Tests and calculations show the error is less than $1 \mathrm{ppm}$ at $50 \mathrm{kHz}$. However, it also permits heat to flow freely from the resistor, which dissipates up to five watts on the $1000-\mathrm{V}$ range, to the TE which has a temperature coefficient of emf of approximately 0.2 percent $/{ }^{\circ} \mathrm{C}$ (at constant input current).

Heat conduction through the center conductor of this connector was reduced, at $300 \mathrm{~V}$ and higher, by replacing the two polystyrene insulators in the coaxial connectors (one in the resistor output and one in the TE input) with two made of boron nitride. This material is a good electrical insulator and yet it conducts heat well enough to provide an effective heat sink for this conductor. It is used in both ends of the high voltage resistors to improve the heat flow from the resistor to the relatively heavy brass casing. This also helps to reduce the temperature rise in the resistor.

Heat flow through the outer part of these connectors will gradually raise the temperature of the TE casing, and this also causes a heating drift. The increase in temperature of the TE bulb is slowed by mounting it in a short section of brass tubing to increase the thermal mass and this tube is thermally insulated from the TE casing. Thermal compensation is also added by attaching a $10-\Omega$ thermistor to the tube containing the TE and connecting it in series with the TE output. A resistor appropriately chosen to complete the compensation circuit (see Fig. 7 and appendix I) will draw current through the thermistor sufficient to compensate for the temperature increase.

This resistor value may be computed for TEs with average temperature coefficients as in appendix I. However, temperature coefficients differ considerably and, for best results, it is usually necessary to match the resistor to the TE and thermistor combination. A suitable value can be found by substituting a variable resistor outside the TE casing and adjusting it for minimum warmup drift on a $1000-\mathrm{V}$ range. However, if the TVC is to be used with a TE comparator similar to the one to be described, it will frequently be connected
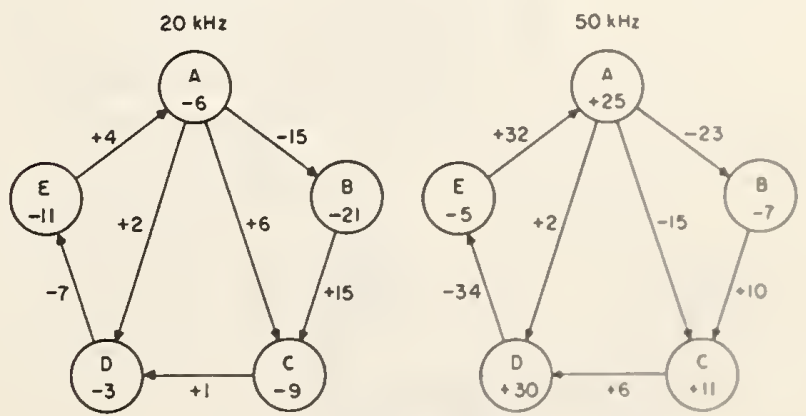

Figure 4. Intercomparisons of 1000-tolt THCs.

Ac-dc difference corrections (ppm) from lable 4 for five lorga. T TV. are ahom the circles and the relative differences frnm table 5 are shown on the interconnortun: lines. The algebraic difference between any pair should be regual to the figure on the connecting line within experimental error. 
across a $1000 \cdot \Omega$ divider, and this will affect the compensation by about 20 percent. A value about 10 per. cent higher than the one chosen experimentally should provide a satisfactory compromise and reduce the drift by a factor of five or more. With the compensation and the large thermal time constant of the TE in its casing, the drift can be reduced to less than $5 \mathrm{ppm} /$ minute under typical laboratory condition. The error from this is almost completely eliminated by taking a sequence of readings with a-c, d-c, reversed d-c, and a-c voltage applied, as described in the next section.

\section{TVC Test Methods}

A brief review of other comparison methods may be useful before describing the TE comparator. Figure 5 shows two TVCs connected in parallel. The separate balancing circuits (or Lindeck Potentiometers) (B) and null detectors (D) may or may not be built in. Stable a-c and $d-c$ voltage supplies are connected to the two TVCs by the ac-dc switch. The switching arrangement shown is typical and convenient, although others, such as reversing the d.c supply by a separate switch, are satisfactory. The d-c supply must have an ungrounded output and it should be possible to ground either terminal as the voltage is reversed. The reversed d-c differences of the TVC can rarely, if ever, be neglected in these tests.

For convenience and clarity, we shall call one TVC the "standard" and the other the "test." Normally the one with known ac-dc difference corrections is the standard, and the test TVC is usually treated differently in the test procedures, as explained below.

There are two procedures for this intercomparison. One might be called a null-balancing method. With a-c voltage applied to both TVCs, the balancing circuits of the test and standard instruments are adjusted to null the detectors. The d-c supply is then switched to the TVCs, and the voltage is adjusted to obtain a null balance first on the detector of the test instrument and then on the standard. Each d-c voltage is carefully measured, and the small difference between the two should equal the difference between the a-c voltage and

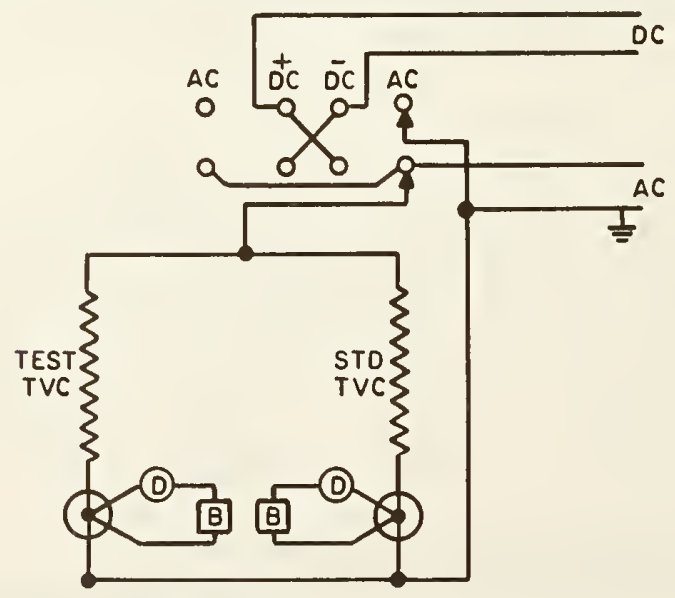

Figure 5. Two-Potentiometer method for TVC comparisnns. one direction of $d \cdot c$ voltage. The measurement is then repeated with reversed $d-c$ voltage, and an average is taken.

This method is tedious and time consuming, an ac. curate $d \cdot c$ voltage standard is required, and if there is any drift in either instrument output due to warmup or other reasons, as there very often is, the data may lack both accuracy and precision.

A more satisfactory procedure and one that will minimize the effect of drift may be called a deflection method. Both balancing circuits are adjusted to near null, and then a-c and d-c voltages are applied in the succession indicated in figure $5(\mathrm{AC}, \mathrm{DC}+, \mathrm{DC}-, \mathrm{AC})$. For each input the power supply is adjusted to set the test instrument detector to null. The deflection of the standard TVC detector is read for each setting, and the difference between the average reading on a-c voltage and $d-c$ voltage is an indication of relative ac-dc difference. If the readings are made at nearly equal time intervals, and if the drift rate is reasonably constant (even though fairly large), the determinations can be made with satisfactory precision.

If the detect or of the standard TVC is a galvanometer the reading may be in divisions or millimeters, but they can be converted to volts or percent ac-dc differences by calibrating the detector scale in one of several ways. A calibration factor can be determined simply and directly by making a small measured change in the $d \cdot c$ input and observing the detector response.

If the detector is a microvoltmeter and if the balancing circuit is a Lindeck potentiometer indicating the millivolt output of the thermoelement, the calibration step can be eliminated. With the four deflection readings on the detector the difference between the average reading on a-c voltage $\left(E_{a}\right)$ and those on d-c voltage $\left(E_{d}\right)$ is computed from the differences of the corresponding readings of the microvoltmeter. The ac-dc difference of the test instrument is

$$
\delta_{t}=\delta_{s}+\frac{E_{s a}-E_{s d}}{n_{s} E_{s d}}
$$

where the subscript $s$ indicates that these values come from the standard instrument, and $\delta_{\delta}$ is the ac-dc difference correction for the standard. ${ }^{5}$

The factor $n$ relates small changes in thermoelement heater current $(\Delta I)$ (at a fixed frequency) to corresponding changes in output emt $(\Delta E / E=n \Delta I / I$ approximately). For a TVC we may substitute $n \Delta V \mid V$ for the right hand expression, so that $n=V \Delta E / E \Delta V$. The value of $n$ is 2 if the thermoelement has a square law response, possibly at very low heater currents, but it is usually 1.7 to 1.9 at rated current. Determinations of $n$ should therefore be made in a d-c test at five or more current levels by measuring the values indicated. A plot of $n$ against $E$ can be made so that values corresponding to any emf can be found for substitution in the equation above (see sec. 8).

\footnotetext{
- See eq 3 of appendix II. This general formula is applicable here also.
} 


\section{TE Comparator}

There are several variations of basic comparator circuits which are used to minimize the difficulty caused by power supply instability $[1,3]$. They employ a voltage divider circuit to which two emfs are connected. The divider is adjusted to null a detector, and at this point the divider setting corresponds to the ratio of the emfs. If one emf $\left(E_{t}\right)$ is held constant as a-c and then d-c voltage is applied to the TVCs, the other emf $\left(E_{s}\right)$ will change if there is a relative ac-dc difference in the TVCs at the frequency $\left(\delta_{t} \neq \delta_{s}\right)$. The resulting unbalance in the divider will produce a change in the detector deflection proportional to the ac-dc difference. However, small fluctuations in the power supply will produce nearly equal proportional changes in the emfs and therefore the detector will not be affected appreciably. The stabilizing effect depends on how well matched the time constants and response characteristics of the TEs are, but the effect usually affords a significant advantage. Also, the monitored emf need not be held constant so exactly as with a simple balancing circuit.

In the new comparator (fig. 6) the higher of the two emfs is connected across a $1000-\Omega$ Kelvin-Varley divider, and the lower one is connected to the variable tap. The detector is brought to null by adjusting the divider. A Lindeck potentiometer and the same detector (with key $k_{1}$ ) is used to monitor the test TVC output $\left(E_{t}\right)$. The detector is labeled " $N$ " in the diagram because the use of a nanovoltmeter is suggested.

The test procedure is similar to that already mentioned. Preliminary settings of the potentiometer and divider are first made so the readings of the nanovoltmeter will fall near mid-scale. Then with a-c voltage applied, the key $\mathrm{k}_{1}$ (labeled "SET" on the instrument, figs. 8 and 9 ) is depressed and the appro-

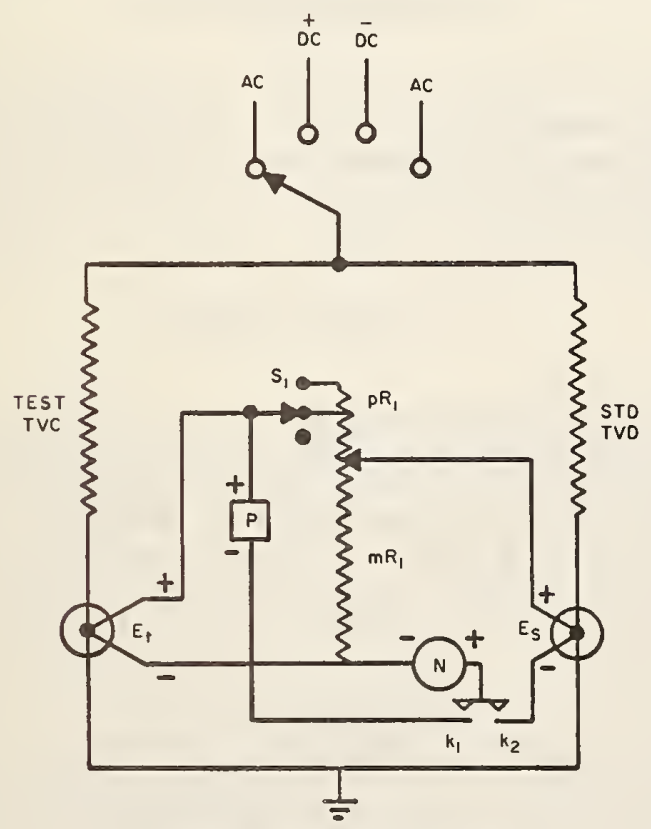

Figure 6. New TE comparator. priate power supply is adjusted for a null on the detector. Key $\mathbf{k}_{\mathbf{2}}$ (READ) is then depressed and the detector indication is read. The two-step procedure is repeated for two directions of $d-c$ voltage and for a second a-c input. The average indication in nanovolts ${ }^{6}\left(N_{a}\right)$ for a-c input and the average for d-c input $\left(N_{d}\right)$ is computed and the ac-dc difference of the test instrument is

$$
\delta_{t}=\delta_{s}+\frac{N_{d}-N_{1}}{n_{s} E_{s}} \quad \text { (appendix II eq 5) }
$$

Figure 6 shows the test TVC output $\left(E_{\ell}\right)$ connected to the high emf terminal ( $\mathbf{E}_{H}$ in fig. 8). If $E_{\ell}$ is smaller than $E_{s}$ it will be necessary to interchange them, but this is a minor inconvenience. If $E_{t}$ is connected to the $E_{l}$, terminal the potentiometer is switched to that input with switch $\mathrm{S}_{2}$ (fig. 8) and $E_{t}$ is monitored as before. The same switch also reverses the polarity of the detector, and therefore the sign of the indication. The slightly modified equation for $\delta_{t}$ is eq 11 in appendix II.

A galvanometer may also be used as a detector but since the circuit resistance is relatively high a photoelectric amplifier is necessary for sufficient sensitivity. The scale must be linear over the portion to be used and a scale calibration is also necessary to relate the readings in divisions or millimeters to a voltage change. The calibration can be made quite easily however by moving switch $S_{1}$ from READ to CAL with $k_{2}$ closed and observing the resulting deflection change. This increases the resistance of the $1000-\Omega$ divider by $0.5 \Omega$ and changes the divider current by 0.05 percent. The
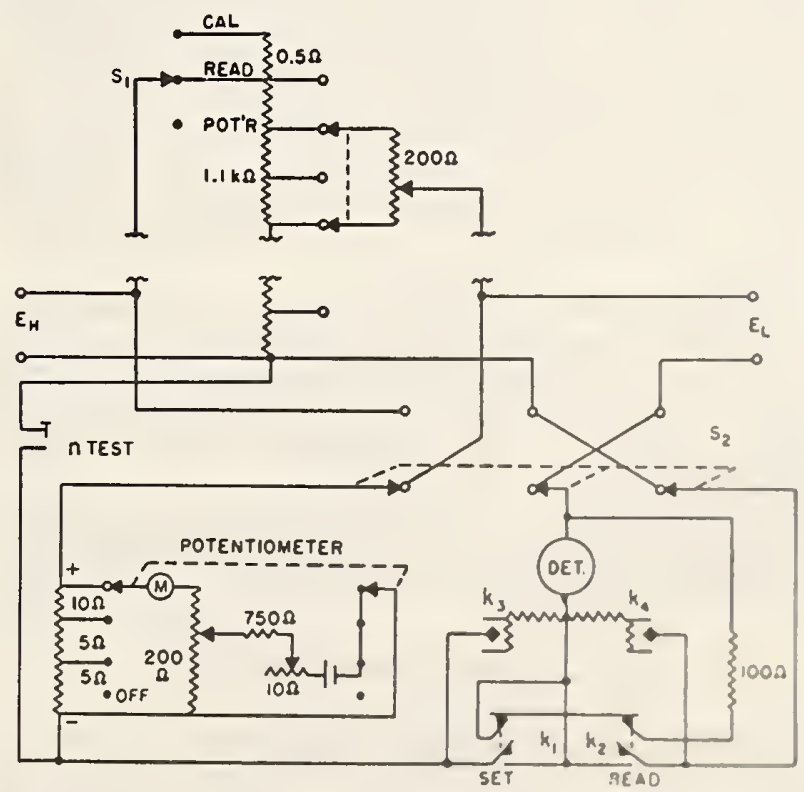

FiGURE 8. New TE compatator 


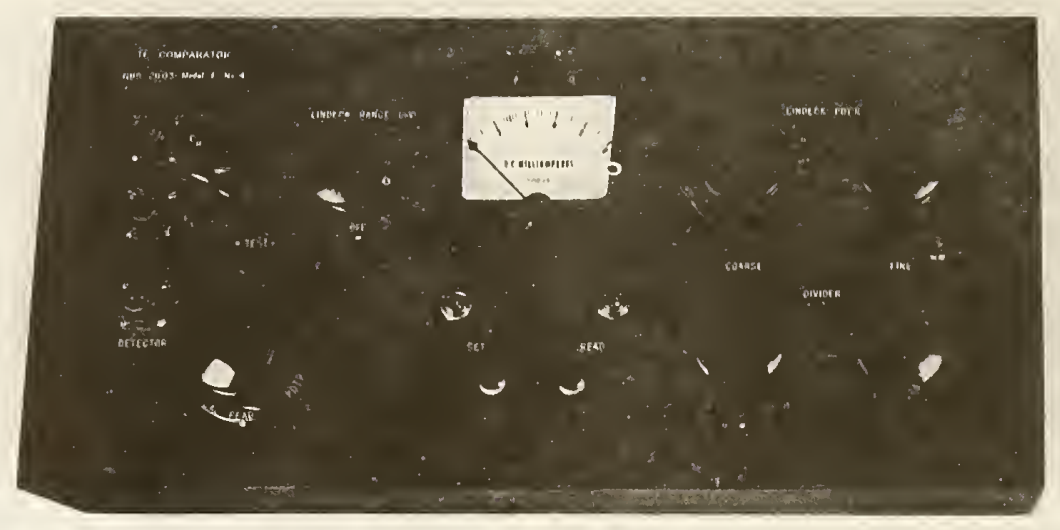

Figure 9. Photogtaph of TE comparator.

voltage applied to the galvanometer circuit is changed by the same amount and the deflection change $D_{1}$ is used in eq 10 and 12 in appendix 2 to determine $\delta_{t}$. A measurement of $E_{s}$ is made only to find the corresponding value of $n_{s}$ from a plot as before.

\section{8. $n$ Tests}

Measurements of $n$ are conveniently made with the comparator. The thermoelement to be tested is connected to the $E_{\mathrm{L}}$ input and the $\mathrm{E}_{\mathrm{H}}$ input is unused. A key labeled " $n$ TEST" (fig. 6) is locked down and switch $S_{2}$ is moved to $E_{H}$ connecting the potentiometer voltage across the divider. The divider is set arbitrarily but preferably near full range and the potentiometer is adjusted to null the detector. The input voltage is then changed by a small measured amount $\Delta V \mid V$, and the resulting change in TE output $(\Delta E)$ is measured by observing the change in indication of the detector (nanovolts or microvolts). ${ }^{7}$ Switch $\mathrm{S}_{2}$ is then moved to $E_{\mathrm{L}}$ and a measurement of TE output $(E)$ is made with the potentiometer. The values for each factor of the equation given above $(n=V \Delta E /$ $E \Delta V)$ are then known and $n$ is calculated from this equation.

If a galvanometer is used as the detector a measurement of $D_{1}$ is made as described above, and the deflection change resulting from the measured change in input voltage $(\Delta V)$ is designated $D_{2}$. Then $n=0.05$ $D_{2} / q D_{1}$, where $q$ is the percentage change in input voltage $(100 \Delta V / V)$.

Determinations of $n$ need be accurate only to a few percent, and, since they are quite stable, retesting is not usually necessary.

\section{AC Voltage Measurements}

As suggested earlier, the comparator may be used with one TVC to measure an a-c voltage such as the

\footnotetext{
${ }^{7}$ A small change in the input to a TVC can be measured with a volt box and potenti ometer or by a setting with a calibrated $d \cdot c$ voltage supply. It is also convenient to use resistor with a shorting s witch in series with a TVC to introduce a small current change $(\Delta /)$ in the thermoelement beater (i.e., $20 \Omega$ with a $40-\mathrm{k} \Omega, 200-V$ TVC will give $100 \Delta / / l=$ 0.05 percent which is equivalent to $100 \Delta V / V)$
}

input to a digital voltmeter or the output of a calibrated a-c voltage supply. Switch $S_{1}$ has a third position marked "POT' $R$ " which disconnects the divider from the $E_{H}$ input. If a TVC output is connected to $E_{H}$, and switch $S_{2}$ is moved to that input, the potentiometer and detector can be used to measure the TVC output and changes in it. The circuit is as figure 5 without the test TVC. The d-c voltage is accurately measured and nom. inally equal to the a-c voltage.

It is suggested that d-c voltage be applied to the TVC first and a detector reading taken. Then, at nearly equal time intervals, a second reading is taken with a-c voltage and a third with reversed d-c voltage. The difference between the average of the readings with a d-c voltage $\left(E_{d}\right)$ and the one with a-c voltage $\left(E_{a}\right)$ is related to the input voltage difference very much as before.

$$
V_{\mathrm{ac}}=V_{\mathrm{dc}}\left(1+\frac{E_{a}-E_{d}}{n E}+\delta_{s}\right)
$$

With a nanovoltmeter the emfs are read directly, but if the detector is a galvanometer a scale sensitivity factor must be determined.

\section{Design Details}

The case of the TE comparator has thermal insulation and electrostatic shielding, and the comparator is carefully built to minimize thermal emfs and contact resistances. The switch decks of the Kelvin-Varley divider and $S_{1}$ and $S_{2}$ have enclosed silver contacts and the keys $k_{1}$ and $k_{2}$ are of a low-thermal type. The back contacts on these keys connect a $100-\Omega$ shunt across the detector to reduce sensitivity and noise until one key or the other is depressed. The fine control for the divider is a ten-turn helical resistor with a special towthermal sliding contact. The resolution of the uncalibrated divider is not quite sufficient for setting an exact null on the detector, but setting within a few divisions is adequate in the ordinary use of the instrument. 


\section{Conclusion}

The use of one resistor unit for two TVC voltage ranges has reduced by about half the time required, over previous TVC sets, to make a complete intercomparison test. Changes in any member of the set are readily detected since frequent intercomparison tests are more feasible.

The TE comparator combines the stabilizing feature of other comparators with much of the simplicity of the single Lindeck potentiometer or balancing circuit. It is relatively inexpensive to build and calculations are simplified and direct, especially where the detector is a nanovoltmeter. Repeated tests have shown that the imprecision is less than 2 ppm and ac-dc transfer accuracies, with the TVC set, are $10 \mathrm{ppm}$ at audio frequencies and $20 \mathrm{ppm}$ up to $50 \mathrm{kHz}$.

This work was supported in part by the Army Metrology and Calibration Center, Redstone, Alabama. Their encouragement and support are gratefully acknowledged. The author also acknowledges the help of C. B. Childers and A. G. Perrey who made many of the exacting measurements described here.

\section{References}

[1] Hermach, F. L. and Williams, E. S., Thermal converters for audiofrequency voltage measurements of high accuracy, IEEE Transactions on Instrumentation and Measurement, IM-15, No. 4, 260-268, (Dec. 1966).

[2] Hermach, F. L. and Williams, E. S., Thermal voltage converters for accurate voltage measurements to 30 megacycles per second, Trans. AIEE (Communication and Electronics), 79, Pt. I, 200-206. (July 1960).

[3] Turgel, Raymond S., A comparator for thermal ac-dc transfer standards, ISA Transactions, 6, No. 4, 286-292, (1967).

\section{Appendix I}

The temperature coefficient of emf, $\beta$, of the average $\mathrm{TE}$ is about -0.2 percent $/{ }^{\circ} \mathrm{C}$, with constant input current. Thus a corresponding decrease in the resistance, $R=R_{c}+R_{\ell}+R_{s}$ (see fig. 7) is required to maintain a constant voltage across the resistor $R_{s}$. For a 1 degree increase in temperature, the

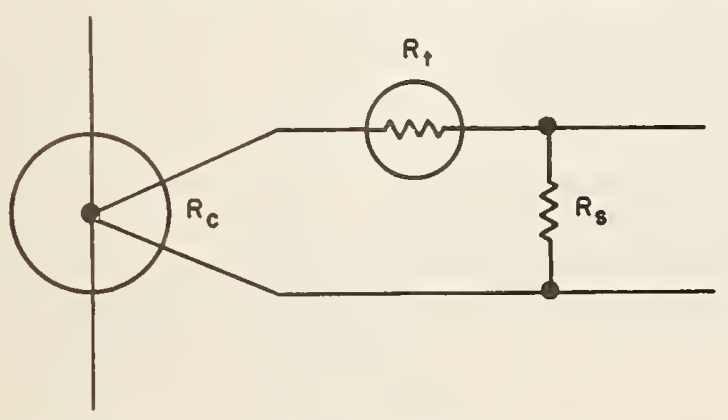

FIGURE 7 . Therma
$R_{\mathrm{c}}$ TE output resistance (about $10 \Omega$ ).
$R_{\mathrm{f}}$ Thermistor resistance (about $10 \Omega$ ).

$R_{\text {}}$ Series resistor to complete compensation circuit. $\left(R_{\epsilon}\right.$ and $R_{\text {, have negligible }}$ temperature coefficients.) change in thermistor resistance, $\Delta R_{t}=\alpha R_{t} / 100$ where $\alpha$ is the temperature coefficient of the thermistor in percent $/{ }^{\circ} \mathrm{C}$-typically about -4 percent $/{ }^{\circ} \mathrm{C}$. Therefore, for thermal compensation,

$$
\begin{gathered}
\beta=100 \Delta R_{t} / R=\alpha R_{t} / R \\
\text { and } R=\alpha R_{t} / \beta
\end{gathered}
$$

With the typical values listed we find $R=200 \Omega$, and, subtracting $R_{c}$ and $R_{\ell}$, the value for $R_{s}$ is $180 \Omega$.

\section{Appendix II}

\section{Development of Equations"}

The ac-dc difference of a TVC is defined as

$$
\delta=\frac{V_{a}-V_{d}}{V_{d}}
$$

where $V_{a}$ is the a-c voltage and $V_{d}$ the average of the two directions of dc-voltage required to produce the same output emf. In the circuit of figure 6 the a-c and $\mathrm{d} \cdot \mathrm{c}$ voltages are adjusted to give the same emf $E_{t}$, of the TVC under test, as indicated by a null on the detector $N$ with $\mathrm{k}_{1}$ closed. The same voltages are applied to the standard TVC. Then,

$$
V_{a}=V_{d}\left(1+\delta_{t}\right)=V_{d}^{\prime}\left(1+\delta_{g}\right)
$$

where $V_{d}^{\prime}$ is the d-c voltage required to produce the same output emf of the standard as $V_{a}$, and the subscripts, $t$ and $s$, refer to the test and standard instruments respectively. If $\frac{V_{d}^{\prime}-V_{d}}{V_{d}}<<1$, then, closely
enough

$$
\delta_{t}-\delta_{s}=\frac{V_{d}^{\prime}-V_{d}}{V_{d}}
$$

From the definition of $n$ given in the paper

$$
\delta_{t}-\delta_{8}=\frac{E_{8 a}-E_{8 d}}{n_{8} E_{8 d}}
$$

With the polarities as shown in figure 6 ,

$$
N=m E_{t}-E_{s}
$$

where $N$ is the detector voltage with $\mathrm{k}_{2}$ closed and $\mathrm{k}_{1}$ open, and $m$ is the divider ratio (with the detector resistance $R_{d}>R_{1}$ ).

Thus.

$$
\delta_{t}=\delta_{s}+\frac{N_{d}-N_{a}}{n_{s} E_{s d}}
$$

where the subscript $a$ and $d$ have the same meaning as before.

\footnotetext{
" These equations were developed by F, $L_{\text {. }}$ Hermech.
} 
If a galvanometer is used as the detector, the current through it with $k_{2}$ closed is, by Thevenin's Theorem,

$$
I_{g b}=\frac{m E_{t}-E_{8}}{R_{e}}
$$

where $R_{e}$ is the resistance of the galvanometer circuit with $E_{t}$ and $E_{R}=0$. With $E_{t}$ and $E_{\varepsilon}$ held constant and $p R_{1}$ inserted by means of $\mathrm{S}_{1}$,

$$
I_{g c}=\frac{m E_{t} /(1+p)-E_{8}}{R_{e}^{\prime}}
$$

where $R_{e}^{\prime}$ is the resistance of the galvanometer circuit.

Since $m E_{t}-E_{s}<<E_{s}$ and $p<<1$, we have to a sufficient degree of approximation,

$$
I_{g b}-I_{g c}=\frac{E_{s} p}{R_{e}}=\frac{\left(D_{b}-D_{c}\right)}{S}=\frac{D_{1}}{S}
$$

where $D_{b}$ and $D_{c}$ are the resulting galvanometer deflections and $S$ is the galvanometer current sensitivity.

In the ac-dc test, with $m E_{\ell}$ constant,

$$
I_{g a}-I_{g d}=\frac{E_{s d}-E_{s a}}{R_{e}}=\frac{D_{a}-D_{d}}{S}
$$

Thus, from (3), (8) and (9),

$$
\delta_{t}=\delta_{s}+\frac{p\left(D_{d}-D_{a}\right)}{n_{s} D_{1}}
$$

The test and standard TVCs may be interchanged if $E_{t}<E_{s}$. If $E_{t}$ is applied to the $\mathrm{E}_{\mathrm{L}}$ input of figure 8 and held constant, with the detector and potentiometer con. nected as shown in the figure, a similar analysis leads to the following equations:

$$
\delta_{t}=\delta_{\mathrm{s}}+\frac{N_{d}-N_{a}}{n_{s} E_{t}}
$$

and

$$
\delta_{t}=\delta_{8}+\frac{p\left(D_{d}-D_{a}\right)}{n_{8} D_{1}}
$$

The characteristic $\boldsymbol{n}$ is determined from

$$
n=\frac{\Delta E / E}{\Delta V / V}
$$

by applying known changes in input voltage and observing the changes in output emf with a high resistance voltmeter, as described in the text. If a galvanometer is used as the detector instead of the voltmeter its sensitivity is determined by inserting $p R_{1}$ as described. From equations similar to (8) and (9)

$$
\frac{\Delta E_{s}}{E_{s}}=\frac{p \Delta D}{D_{1}}
$$

If the thermocouple resistance of the test TVC, $R_{t c}$, is significant, $p$ in equation (7) should be replaced by $p^{\prime}$, the fraction of $R_{1}+R_{t c}$ inserted by $S_{1}$. Similarly if the potentiometer resistance $R_{p}$ is significant, $p$ in equation (14) should be replaced by $p^{\prime \prime}$, the fraction of $R_{1}+R_{p}$ inserted by $\mathrm{S}_{1}$.

(Paper 75C3\&4-321)

* with a high impedance detector. With a galvanometer the formula for $\delta_{t}$ is too complicated to be usefur. 


\title{
An Investigation of Multijunction Thermal Converters
}

\author{
FRANCIS L. HERMACH, FELLOW, IEEE, AND DONALD R. FLACH
}

\begin{abstract}
The relative ac-de differences of a group of multijunction thermal converters (MJTC's) have been determined over the frequency range $30 \mathrm{~Hz}-10 \mathrm{kHz}$. These MJTC's are of different ranges and were obtained from several sources. Differences were observed at low frequencies when converters of various ranges were intercompared. For voltage measurements, the use of matched reaistors in series with the MJTC heater resistors greatly reduced these errors and contributed to the reduction of other errors as well. It is believed that the average ac-dc difference of this group is less than $0.3 \mathrm{ppm}$ at $160 \mathrm{~Hz}$ and $0.5 \mathrm{ppm}$ up to $10 \mathrm{kHz}$.
\end{abstract}

\section{INTRODUCTION}

$\mathbf{M}$ FASUREMENTS of rms current and voltage have been based on a technique which converts electrical energy into heat and compares this with the heat produced by a dc signal. Single-junction thermal converters, each consisting of a wire heated by an electric current with a thermocouple to sense the temperature-rise, have been in use for almost 3 decades at NBS for accurate rms ac-dc transfer measurements. Recently, a potentially more accurate form of the resistor-thermocouple combination, the multijunction thermal converter (MJTC), was developed at NPL by Wilkins [1]. Verification of these MJTC's, to $0.5 \mathrm{ppm}$ at $9 \mathrm{~V}$ and $159 \mathrm{~Hz}$, was initially needed at NBS for an absolute volt experiment, now well underway. Later, a goal of $1 \mathrm{ppm}$ from $30 \mathrm{~Hz}$ to $10 \mathrm{kHz}$ was established as a marked and useful advance over the present $10 \mathrm{ppm}$.

Each MJTC has a bifilar heater wire with from 50 to 200 series-connected thermocouples to sense the heater current, overcoming fundamental limitations in single-junction thermal converters [2]. Since actual converters may depart from the ideal values, experimental comparisons of converters of different construction, range, etc are desirable to buttress the thenretical accuracy. Unfortunately there are at present no other suitable kinds of ac-dc transfer standards to provide additional verification [3].

MJTC's with heaters ranging from 5 to $25 \mathrm{~mA}$ and with rated output EMF's of 30 to $120 \mathrm{mV}$ were obtained from commercial manufacturers for this investigation, and 50-mA MJTC's were donated by NPL. Techniques were developed for intercomparing MJTC's with high precision, and a number of ac-dc errors were uncovered and overcome, to attain the desired goals.

\section{EMF COMPARATOR}

The ac-dc difference is defined as $\delta=\left(Q_{a}-Q_{d}\right) / Q_{d}$ where $Q_{a}$ and $Q_{d}$ are the ac and dc inputs which produce

Manuscript received June 29, 1976.

'The authors are with the Electricity Division, National Bureau of Standards, Washington, DC 20234. the same output of a thermal converter. A modified Williams EMF comparator [4] was used with a commercial chopper-type nanovoltmeter to determine $\Delta=\delta_{1}-\delta_{2}$, for two MJTC's whose heaters were connected either in series as thermal current converters (TCC's) or in parallel as thermal voltage converters (TVC's). A simplified schematic of the measuring circuit is shown in Fig. 1. The 11-k $\Omega$ three-stage divider $D$, (adjustable to $1 \mathrm{ppm}$ ) and the Lindeck potentiometer $P$, were first adjusted to bring the nanovoltmeter $N$, near zero for both positions of the key $K$ with dc applied, and were thereafter not disturbed during an ac-dc determination. $S$ was switched to ac, dc-D (direct), dc-R (reversed), and AC in succession. In each case the supply voltage was adjusted to bring $N$ to zero ${ }^{1}$ in the $A$ position of $K$ and the reading of $N$ in the $B$ position was recorded. As shown in [4], $\Delta=\delta_{1}-\delta_{2}=\left(N_{d}-\right.$ $\left.N_{a}\right) / n_{2} E_{2}$, where $N_{a}$ and $N_{d}$ are the average voltage readings with ac and the two directions of dc applied, respectively, and $n_{2}$ is 2 for these square-law MJTC's.

When the dc reversal difference was larger than the range of $N$, the reversal compensator (RC) was used. It consisted of an adjustable, $0-13-\mu \mathrm{V}$ dc source, with negligible change on reversal. It was switched on for one direction of the applied dc current and reversed for the other, so that there was no net change in the average.

Carefully timed sequences of ac-dc readings, with $30-\mathrm{s}$ intervals and less than 10 -ms off times were necessary to overcome serious limitations from drifts and fluctuations in the MJTC's as well as the power supplies. In some cases Johnson noise was $0.3 \mathrm{ppm}$, and detector noise was 0.6 ppm, peak to peak, in the pass band of the detector. The MJTC's were placed in a $0.02 \mathrm{~m}^{3}$ passive oil bath in a temperature controlled room. Even so, some had to be discarded because of excessive drifts or large switching transients.

The detector had a $20-\mathrm{dB}$ narrow-band rejection filter centered at $60 \mathrm{~Hz}$, and was sensitive to common-mode voltage at and near harmonics of its chopper frequency of $94 \mathrm{~Hz}$. Significant ac outputs at twice the input frequency ${ }^{2}$ were observed in MJTC's below $100 \mathrm{~Hz}$ (approximately proportional to $1 / f^{1.5}$ ), and at the input frequency above $1 \mathrm{kHz}$. Errors from these were reduced by modifying the filter and connecting a $2-\mu \mathrm{F}$ capacitor across the instrument.

To verify the accuracy of the nanovoltmeter, a number of ac-dc difference measurements were also made with a

\footnotetext{
'Close settings were not necessary for square law M.JTC"s like the prototype the potentiometer could be connected across $E_{\%}$, but thin was rarely done, except to measure $E_{2}$.

2 Peak-to-peak ar outputs of up to 0.5 percent, over 1000 tumen the normally used $0.3-\mu \mathrm{V}$ range of the detector, were observed at in input frequency of $30 \mathrm{~Hz}$.
} 


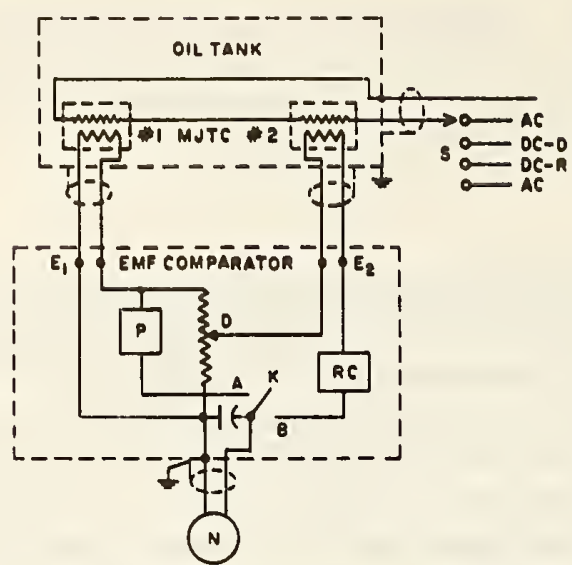

Fig. 1. Comparison circuit.

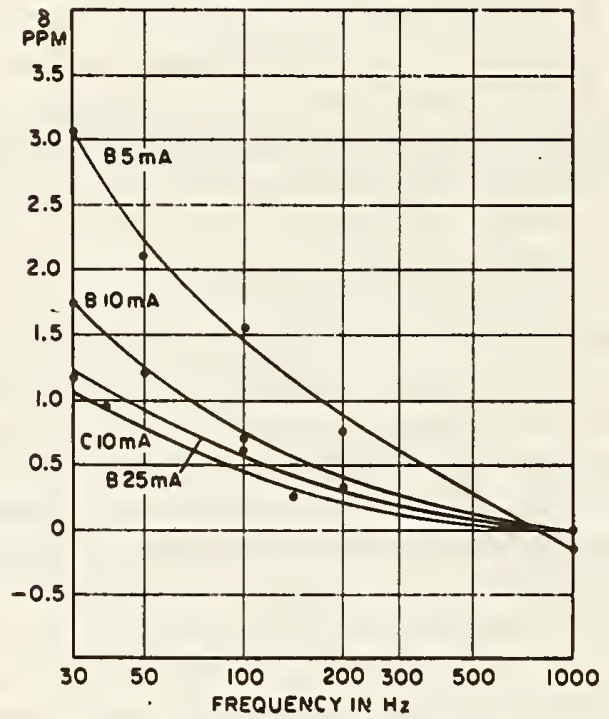

Fig. 2. Ac-dc differences of TCC's.

photocell galvanometer amplifier as a null detector, inserting small known voltages in the detector circuit with the reversal compensator. The results with the two detectors were in agreement to $0.2 \mathrm{ppm}$ or better.

\section{LOW-FREQUENCY ERRORS}

Initial tests were made from 30 to $1000 \mathrm{~Hz}$ (a range near $5 / 1$ about $160 \mathrm{~Hz}$ ). Stepup icomparisons as thermal current converters (TCC's) were made by connecting, for example, two 5-mA MJ̣TC's in parallel (outputs in series) and connecting the pair in series with a 10-mA MJTC. The two 5-mA MJTC's were also compared, with their heaters in series. These tests disclosed alarming differences between ranges, roughly inversely proportional to frequency, as shown (at rated currents) in Fig. 2, with the $50 \mathrm{~mA}$ MJTC's taken as a base. The cause of these differences is as yet unknown. The error was affected by shunting the heater and was opposite in sign for TVC's. Thus it was caused by a change in the effective resistance of the heater at low frequencies.
As the frequency of the applied current decreases, the heater temperature of a thermal converter begins to follow the double-frequency variation of the instantaneous input power. This can cause low-frequency errors if the resistance is dependent on the temperature. However, the MJTC heaters are quaternary alloys of mainly $\mathrm{NiCr}$, which have low temperature coefficients $\left(0 \pm 20 \mathrm{ppm} /{ }^{\circ} \mathrm{C}\right)$. One pair of MJTC's of the same range had nearly equal lowfrequency errors but opposite temperature coefficients. The error was generally opposite in sign from that deduced mathematically by treating all of the heater as a mass at uniform temperature, with all of the heat lost by temperature-independent lateral cooling. The thermal situation must be much more complex than this, as evidenced by the measured $1 / f^{1.5}$ variation of ac output with heater frequency, instead of $1 / f$ as predicted by this and other simple models when the ratio of ac to dc outputs is small.

A prototype MJTC with a $\mathrm{NiCr}$ heater from a third manufacturer had much larger ac dc differences; about $-100 \mathrm{ppm}$ as a TCC and $+120 \mathrm{ppm}$ as a TVC at $30 \mathrm{~Hz}$. This shows rather strikingly the necessity for experimentally verifying new designs. These errors agreed with the sign predicted by the model, but varied roughly as $1 / f$ instead of $1 / f^{2}$ as predicted for low ac outputs.

Ac bridge measurements showed that the low-frequency insulation resistance between the halves of a heater that had been cut in the middle was too high to account for the low-frequency error. Thus the low-frequency error is apparently not caused by the temperature coefficient of resistance of the heater or dielectric loss between the halves of the heater.

A well-known theorem states that the power in a resistor $R_{1}$ fed by a constant voltage source having a fixed internal resistance $R_{2}$ is a maximum when $R_{1}=R_{2}$. From this, as shown in the appendix, the power in the heater of an MJTC used to measure voltage would to the first order be independent of small changes in the heater resistance if a matched (equal) fixed resistor were connected in series with the heater. Analogously, for measuring current the power would be independent of small changes with a matched resistor across the heater. Since the thermocouples respond to the power dissipated in the heater, these interesting applications of the maximum-power-transfer theorem make possible compensated MJTC's, in which the first-order errors from changes in effective resistance are eliminated, at the cost of doubling the current and voltage ranges. Calculations and tests showed that matching to 10 percent is adequate for the desired accuracy.

\section{OTHER ERRORS}

Other errors in voltage measurements arise from Peltier effects at the junctions between the heater and its lead-in wires, and skin effect in magnetic leads used in some MJTC's [1], [2]. For a given construction the errors from Peltier effects are independent of frequency (except at low frequencies) and current level, and are inversely proportional to the resistance, so that they could in principle be 
TABLE I

TVC Comparisons

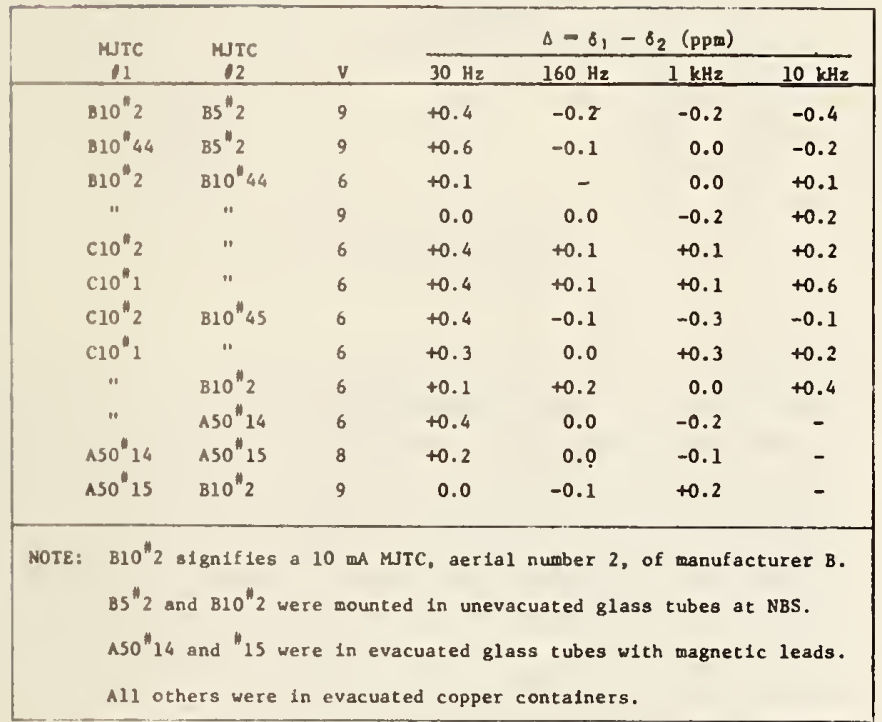

evaluated by making TVC tests with different series resistors. However the low-frequency variation can be complicated, and the evaluation is difficult in the presence of other errors. Instead these and skin-effect errors were eliminated by specifying MJTC's with copper leads, which have low thermal emfs against the quaternary heater alloys.

At higher frequencies (up to $10 \mathrm{kHz}$ ), tests disclosed errors in some of the MJTC's from dielectric losses between the heater and the thermocouples. ${ }^{3}$ For example, differences of up to several ppm at $10 \mathrm{kHz}$ could be obtained by changing the ground from one line to the other in tests of two 5-mA TCC's in series. Dielectric errors were also greatly reduced by the matched resistors. (See Appendix.)

\section{TVC TESTS}

Since the needs were for voltage measurements, a group of $8 \mathrm{MJTC}$ 's of 4 different constructions and 3 current ranges, and with copper leads, were compensated with matched series resistors of quaternary alloys wound on thin mica cards. ${ }^{4}$ Each MJTC with its resistor was mounted in an aluminum box (through which oil could circulate), with input binding posts and a 2-pin output connector of low thermal EMF's. They were compared as TVC's at 6 to $9 \mathrm{~V}$, at $30,160,1000$, and $10000 \mathrm{~Hz}$. The results are shown in Table I.

At the important frequency of $160 \mathrm{~Hz}$ the results are shown more vividly in Fig. 3. The circles identify the MJTC's and the number adjacent to each arrow gives $\delta_{h}$ - $\delta_{t}$ in ppm, where $\delta_{h}$ and $\delta_{t}$ are, respectively, the ac-dc

3 Dielectric losses in the oil were negligible.

${ }^{4}$ Two MJTC's, with 50-mA ranges, have magnetic leads and were not used above $1 \mathrm{kHz}$. A series resistor of $200 \Omega$ was used with each, to give a $10-V$ range. Tests with other resistors showed that resistance-dependent low-frequency errors of these MJTC's were less than $0.5 \mathrm{ppm}$. Series resistors for the other MJ'TC's were within 10 percent of the heater resistances.
TABLE II

Assigned AC-DC Differences

\begin{tabular}{|c|c|c|c|c|}
\hline \multirow[b]{2}{*}{ MJTC } & \multicolumn{4}{|c|}{$\delta(\mathrm{ppm})$} \\
\hline & $30 \mathrm{~Hz}$ & $16 n \mathrm{~Hz}$ & $1 \mathrm{kHz}$ & $10 \mathrm{kHz}$ \\
\hline$B 10^{\#} 44$ & 0.0 & -0.1 & -0.1 & -0.2 \\
\hline$B 10^{\#} 45$ & 0.0 & +0.1 & -0.1 & 0.0 \\
\hline $\mathrm{C} 10^{*} 1$ & +0.3 & +0.1 & 0.0 & +0.3 \\
\hline $\mathrm{C} 10 \# 2$ & +0.4 & 0.0 & -0.2 & 0.0 \\
\hline В $10 * 2$ & 0.0 & -0.1 & -0.1 & -0.2 \\
\hline $\mathrm{BS}^{\mathrm{H}} 2$ & -0.5 & +0.1 & 0.0 & +0.1 \\
\hline $\mathrm{A} 50^{\# \prime} 14$ & 0.0 & 0.0 & +0.2 & - \\
\hline A 50 " 15 & -0.1 & -0.1 & +0.2 & - \\
\hline
\end{tabular}

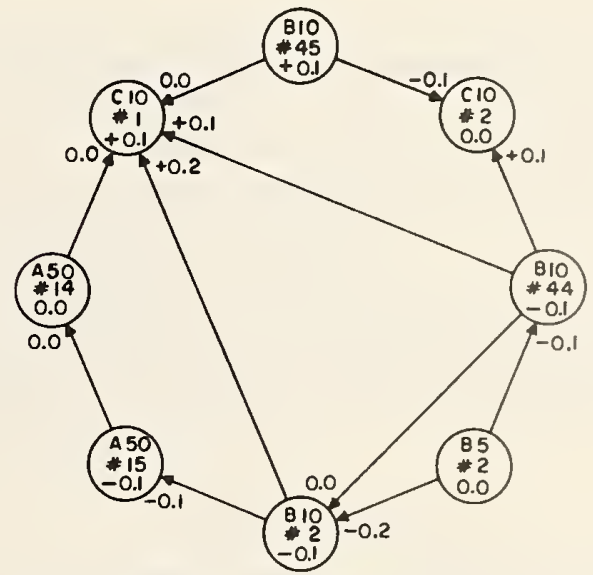

Fig. 3. TVC's at $160 \mathrm{~Hz}$.

differences of the MJTC's at the head and tail of the arrow. In the absence of systematic errors the arrows around the loops should sum to zero within the limits imposed by the random errors of the comparisons. Alternatively failure to close properly is an indication of systematic errors. Techniques described by Youden [5] can be used to eval. uate networks such as this.

The values of $\delta$ assigned in this way to each MJTC at 160 $\mathrm{Hz}$ are shown in each circle, on the assumption that the average of all is zero. Each value is less than $0.2 \mathrm{ppm}$, and the closure errors are very small.

Similar analyses were carried out for the measurements at other frequencies. The assigned ac-dc differences are shown in the columns marked $\delta_{v}$ in Table II. The discrepancies are somewhat larger than at $160 \mathrm{~Hz}$ but there is no evidence of systematic error, or of significant residual low-frequency errors in these compensated MJTC's.

The standard error $s$ (standard deviation of the average of 4 determinations) in a comparison of 2 M.JT'C's was 0.12 ppm. Because of the network of comparisons the standard deviation of an MJTC with respect to the average should be somewhat less than this.

\section{SERIFS RESISTORS}

The high-quality wire-wound series resistors used with these iMJTC's have excellent ac-dc characteristics at audio frequencies. The phase angles are small and cause only a 



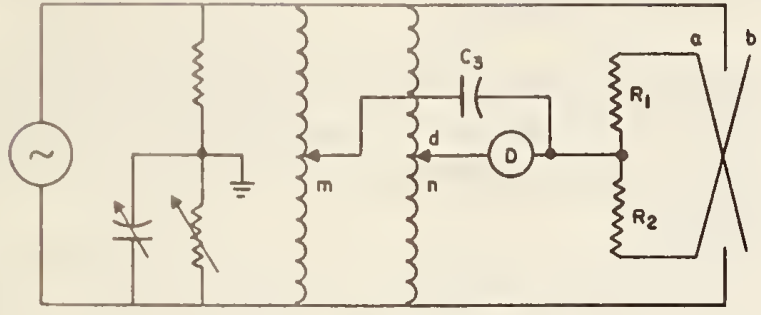

Fig. 4. Reversing bridge.

second-order effect on impedance magnitude. However there is a possibility of dielectric loss in the insulation at the higher audio frequencies. (The maximum powertransfer theorem does not help here.) To determine this, the $1 / 1$ inductive-voltage-divider bridge shown in Fig. 4 was set up to compare two nearly equal resistors $R_{1}$ and $R_{2}$. A balance was made for each switch position of this "reversing bridge" by adjusting $m$ and $n$ with the detector lead $d$, as shown, after adjusting the Wagner arms with $d$ grounded. Most of the bridge errors were eliminated in this way, leaving as the difference equations, $\left(R_{2}-R_{1}\right) / R_{1}=$ $2\left(n_{a}-n_{b}\right)$ and $\alpha_{2}-\alpha_{1}=\gamma\left(m_{a}-m_{b}\right)$ where $R$ and $\alpha$ are the resistive components and the phase angle, respectively, of the parallel equivalent circuit for each resistor, $\gamma=$ $\omega C_{3} R$, and the subscripts $a$ and $b$ refer to the switch positions shown. These simple equations hold to $0.1 \mathrm{ppm}$ if the phase angles of $C_{3}$ and $m$ are each less than $0.1 \mathrm{mrad}$ and $\left(\alpha_{2}-\alpha_{1}\right)$ is less than 1 mrad. $^{5}$

A $1-k \Omega$ resistor of the same type as those used with the MJTC's was mounted in a metal box, for four-terminalpair ac-dc measurements on Cutkosky's bridges [6] from 160 to $16000 \mathrm{~Hz}$. This served as a standard for evaluating two $1-\mathrm{k} \Omega$ unmounted resistors. These were paralleled to evaluate a 5 -section $500-\Omega$ card. The 5 sections were intercompared and then used to evaluate the 300,400 , and $500-\Omega$ cards for the MJTC's. The $1300-\Omega$ card for the $5-\mathrm{mA}$ MJTC was compared with the $1-\mathrm{k} \Omega$ card in series with 3 $100-\Omega$ sections.

An upper limit of $10 \mathrm{kHz}$ was imposed by the effects of lead inductance and of stray capacitance from the cards to the grounded aluminum boxes in which they were tested and used. Below this, changes in the parallel resistances were less than $0.1 \mathrm{ppm}$ for drastic changes in the positions of the resistors. The cases of the inductive voltage dividers and the shields of all leads of the reversing bridge were grounded. $C_{3}$ was a low-loss 3 -terminal air capacitor, and $D$ was a tuned null detector. All bridge components were commercially available.

Measurements were made at $0.1,1$, and $10 \mathrm{kHz}$ with 8 $V$ applied. The results are shown in Table III as $\delta_{e}$ and $\alpha$, where $\delta_{e}=\left(R_{f}-R_{p}\right) / R_{p}$ and $\alpha$ is the phase angle. $R_{f}$ and $R_{p}$ are the parallel-equivalent-circuit resistances at the frequency $f$ and the base frequency $(0.1 \mathrm{kHz})$, respectively. For each resistor, $\alpha$ was closely proportional to $f$, and so is not shown at $1 \mathrm{kHz}$ in the table.

${ }^{5}$ The dividers were rated up to $10 \mathrm{kHz}$. Only the lower dials were changed on reversal, with only moderate accuracy required.
TABLE III

Resistance Measurements

\begin{tabular}{|c|c|c|c|c|c|c|}
\hline \multirow[b]{2}{*}{ MJTC } & \multirow{2}{*}{$\begin{array}{l}\mathbf{R}_{8} \\
\Omega\end{array}$} & \multirow{2}{*}{$\frac{1 \mathrm{kHz}}{\delta_{e}}$} & \multicolumn{4}{|c|}{$10 \mathrm{kHz}$} \\
\hline & & & $\delta_{e}$ & $\alpha$ & ${ }^{\delta_{r}}$ & ${ }_{{ }^{8}}{ }_{c}$ \\
\hline $810^{\#} 44$ & 500 & -0.1 & -0.7 & -0.55 & -0.5 & +0.3 \\
\hline $810^{*} 45$ & $"$ & +0.1 & +0.3 & -0.41 & +0.1 & -0.1 \\
\hline $\mathrm{C} 10^{\# 1}$ & 300 & 0.0 & +0.5 & -0.60 & +0.1 & +0.2 \\
\hline $\mathrm{ClO}{ }^{\mathrm{B}} 2$ & $"$ & 0.0 & 0.0 & -0.41 & -0.1 & +0.1 \\
\hline$B 10 " 2$ & 400 & 0.0 & +0.4 & -0.47 & +0.1 & -0.3 \\
\hline$B 5{ }^{\prime \prime} 2$ & 1300 & 0.0 & 0.0 & -0.26 & 0.0 & +0.1 \\
\hline
\end{tabular}

NOTE: All values of $\delta$ in Ppm, a in mrad. See text

The effect of these small errors on the magnitude of the current through the MJTC is approximately $\delta_{r}=\delta_{e} / 2-$ $3 \alpha^{2} / 8$. This was negligible at $1 \mathrm{kHz}$ and, except for one resistor, even at $10 \mathrm{kHz}$. The average for all of the resistors was less than $0.1 \mathrm{ppm}$ at each frequency, so that no corrections need be applied to Table II.

The ac-dc differences of a TVC is $\delta_{v}=\delta_{r}+\delta_{c}$ [2] where $\delta_{c}$ is the ac-dc difference of the thermal converter. Since $\delta_{v}$ and $\delta_{r}$ were evaluated as indicated above, $\delta_{c}$ could be determined for each MJTC. As shown in Table III, each was $0.3 \mathrm{ppm}$ or less at $10-\mathrm{kHz}$.

\section{CONCLUSIONS}

This work reaffirms the value of both experimental and theoretical investigations to evaluate ac-dc differences in an absolute sense, and to determine how well áctual ac-dc transfer standards meet the theoretical ideals. A number of sources of ac-dc differences were uncovered, and were eliminated by changes in design, or were compensated for. The agreement between a group of $8 \mathrm{MJTCs}$ which met these requirements was excellent. Because of this, because of the significant differences in construction and ranges of these MJTC's, and because of our long investigation, we believe it is very unlikely that the average ac-dc difference of this group exceeds $0.3 \mathrm{ppm}$ at $160 \mathrm{~Hz}$ or $0.5 \mathrm{ppm}$ at 30 and $10000 \mathrm{~Hz}$. This belief is supported by an analysis based on estimates of the limits of error from such factors as mismatch of series resistors, Peltier-heat exchanges, ac-dc differences of resistors, systematic errors in the comparator, and random errors. For the latter we took as the 95 percent confidence limit $2 s / \sqrt{n}$, where $n$ is the number of MJTC's in the group.

A few measurements over several months indicate a repeatability of $0.3 \mathrm{ppm}$ or better. The long-term stability of ac-dc difference is as yet unknown, but there is no reason to expect drifts greater than this.

Extensions to higher voltages and frequencies seem feasible. Small ac-dc differences in the effective heater resistance could be compensated with combined shunt and series resistors, as indicated in the Appendix. A wide range of film resistors could be mounted in a geometry that would allow the reactance to be calculated, so that the powerful comparison technique of $[2]$ could then be ap plied. 


\section{APPENDIX \\ COMPENSATION FOR CHANGES IN RESISTANCE}

When a resistor $m R$ is in series with MJTC heater, $R(1$ $+a), a \ll 1$, the current (with no reactance) is

$$
I=\frac{V}{(m+1) R(1+a /(m+1))}
$$

$$
\simeq \frac{V}{(m+1) R}\left(1-\frac{a}{m+1}\right)
$$

neglecting higher order terms in $a$. The power in the heater is

$$
P=I^{2} R(1+a) \simeq \frac{V^{2}}{(m+1)^{2} R}\left(1-\frac{2 a}{m+1}+a\right) .
$$

For a fixed $V$ and $m$, the power is independent of $a$ if $m$ $=1$ (neglecting higher order terms). Thus since the thermocouples respond to the power dissipated in the heater, a small difference between the effective dc and ac heater resistances will cause no first-order ac-dc difference in a TVC with a matched series resistor. Matching to 10 percent will reduce a $2 \mathrm{ppm}$ ac-dc difference (from this cause) to $0.1 \mathrm{ppm}$.

Similarly for a TCC with $m R$ in parallel with $R(1+a)$ the power in the heater is independent of $a$ if $m=1$, so that there will be no first-order ac-dc difference with a matched shunt.

This compensation will be effective, however, only if the heater is a two-terminal resistor in the measuring circuit, and if the thermocouples are insensitive to any change in the distribution of the power. For example, an error would occur with dielectric leakage from the heater to the ther- mocouple of an MJTC unless the thermocouple. output and one end of the heater were connected together, and the thermocouple resistance was much less than the insulation resistance.

Matching provides only a single compensated voltage or current range. However, for higher voltage ranges a similar analysis shows that if a resistor $n R$ is connected across the heater and $m R$ in series with the combination, compensation is achieved if $n=m /(m-1)$.

\section{ACKNOWLEDGMENT}

The authors are very grateful to F. Wilkins for the NPL MJTC's, to R. Cutkosky for the ac-dc measurements of the mounted resistor, and to J. Sutcliffe and I. Malcolm of Guildline Instruments Ltd. for their extended cooperation.

\section{REFERENCES}

[1] F. J. Wilkins, "Theoretical analysis of the ac/dc transfer differences of the NPL thermal converter over the frequency range dc-100 kHz," IEEE Trans. Instrum. Meas., vol. IM-21, pp. 334-340, 1972.

[2] F. L. Hermach and E. S. Williams, "Thermal converters for audiofrequency voltage measurements of high-accuracy," IEEE Trans. Instrum. Meas., vol. IM-15, pp. 260-268, 1966.

[3] F. L. Hermach, "Ac-dc comparators for audio-frequency current and voltage measurements," to be published.

[4] E. S. Williams, "Thermal voltage converters and comparators for very accurate ac voltage measurements," J. Res. Nat. Bur. Stand., 75C, pp. 145-154, July-Dec. 1971.

[5] W. J. Youden, "Measurement agreement comparisons," in Proc. 1962 Standards Lab. Conf., NBS Misc. Publ. 248, pp. 147-152, Aug. 1963.

[6] R. Cutkosky, "New NBS measurements of the absolute Farad and Ohm," IEEE Trans. Instrum. Meas., vol. IM-23, pp. 305-309, 1974. 
U.S. OEPT. OF COMM.

BIBLIOGRAPHIC DATA

SHEET (See Instructions)
1. PUBLICATION OR REPORT NO.

NBSIR84-2903
2. Performing Organ. Report No: 3. Publication Date

ApriI 1985

4. TITLE AND SUBTITLE

An Investigation of the Uncertainties of the NBS Thermal Voltage and Current Converters

5. $A U T H O R(S)$

F. L. Hermach

6. PERFORMING ORGANIZATION (If joint or other thon NBS, see instructions)

7. Contract/Grant No.

NATIONAL BUREAU OF STANDARDS

DEPARTMENT OF COMMERCE

8. Type of Report \& Period Covered

WASHINGTON, D.C. 20234

9. SPONSORING ORGANIZATION NAME AND COMPLETE ADDRESS (Street, CHY, Stote, ZIP)

National Bureau of Standards

10. SUPPLEMENTARY NOTES

Document describes a computer program; SF-185, FIPS Software Summary, is attached.

11. ABSTRACT (A 200-word or less factual summary of most significant information. If document includes a significant bibliography or literature survey. mention it here)

The uncertainties of the NBS reference and working standards for ac-dc current and voltage transfer measurements have been redetermined, to 50 and $100 \mathrm{kHz}$, respectively, by means of a set of multijunction thermal converters (MJTCs), an improved emf comparator, and extensive series of intercomparisons. Numerous supporting investigations have also been performed. As a result of this work the accuracy of the NBS standards and the output of its calibration service for ac-dc current and voltage transfer are considered to be on a much firmer and better documented basis than heretofore. Improvement by factors of two to five in the calibration accuracy for high-quality, single-range thermoelements and thermal voltage converters is possible for certain ranges of current, voltage, and frequency. For very special tests, such as international comparisons, accuracies approaching $1 \mathrm{ppm}$ are within reach. (Nineteen references)

12. KEY WOROS (Six to twelve entries; alphabetical order: capitalize only proper names; and separate key words by semicolons) accuracy of ac measurements; ac-dc transfer standards; calibration accuracy; current measurements; measurement accuracy; multijunction thermalconverters; thermal current converters; thermal voltage converters; uncertainties of measurements; voltage measurements 13. AVAILABILITY

X Unlimited

$\square$ For Official Distribution. Do Not Release to NTIS

$\square$ Order From Superintendent of Documents, U.S. Government Printing Office, Washington, D.C. 20402.

14. NO. OF PRINTED PAGES

[X] Order From National Technical Information Service (NTIS), Springfield, VA. 22161
122

15. Price 

